\title{
MIRKOVIĆ-VILONEN POLYTOPES AND A QUIVER CONSTRUCTUION OF CRYSTAL BASIS IN TYPE $A$
}

\author{
YOSHIHISA SAITO
}

\begin{abstract}
In the current paper, we give a quiver theoretical interpretation of MirkovićVilonen polytopes in type $A_{n}$. As a by-product, we give a new proof of the AndersonMirković conjecture which describes the explicit forms of the actions of lowering Kashiwara operators on the set of Mirković-Vilonen polytopes.
\end{abstract}

\section{INTRODUCTION}

1.1. Let $B(\infty)$ be the crystal basis of the negative half of the quantum universal enveloping algebra associated with a symmetrizable Kac-Moody Lie algebra $\mathfrak{g}$. Each element $b \in B(\infty)$ can be written as

$$
b=\tilde{f}_{i_{1}} \tilde{f}_{i_{2}} \cdots \tilde{f}_{i_{k}} b_{\infty}
$$

Here $\tilde{f}_{i}(i \in I)$ is a lowering Kashiwara operator and $b_{\infty}$ the highest weight element of $B(\infty)$. However, for a given $b \in B(\infty)$, the above expression is not unique. For example, the following equality holds in the case of $\mathfrak{g}=\mathfrak{s} l_{3}$ :

$$
\widetilde{f}_{1}^{m} \widetilde{f}_{2}^{m+n} \widetilde{f}_{1}^{n} b_{\infty}=\widetilde{f}_{2}^{m} \widetilde{f}_{1}^{m+n} \widetilde{f}_{2}^{n} b_{\infty} \quad \text { (for any } m, n \in \mathbb{Z}_{\geq 0} \text { ). }
$$

Therefore, in the study of $B(\infty)$, it is important to give

- a parametrization of each element $b \in B(\infty)$ (we call it a realization of $B(\infty)$ ) and

- explicit identifications between several realizations.

Until now, many useful realizations of $B(\infty)$ are known. For example,

(a) a realization in terms of Lakshimibai-Seshadri path (Littelmann [Li]),

(b) a polyhedral realization (Nakashima and Zelevinsky [NZ]),

(c) a Lagrangian (or quiver) realization (Kashiwara and the author [KS]), etc.

These realizations work for arbitrary symmetrizable Kac-Moody Lie algebras. On the other hand, for the case that $\mathfrak{g}$ is a finite dimensional simple Lie algebras, there is

(d) a realization by using the theory of PBW basis (Lusztig [L1], the author [S]).

1.2. Recently, Kamnitzer [Kam1, Kam2] gave a new realization of $B(\infty)$ for the case that $\mathfrak{g}$ is a finite dimensional simple Lie algebras.

Let us explain a background of his work. In several years ago, Mirković and Vilonen introduced a new family of algebraic cycles (called Mirković-Vilonen cycles) in the corresponding affine Grassmannian [MV1, MV2]. Furthermore, Breverman and Gaitsgory [BG] showed that a certain set of Mirković-Vilonen cycles has a crystal structure which is isomorphic to the crystal basis of an irreducible highest weight $U_{q}\left(\mathfrak{g}^{\vee}\right)$-module, where $\mathfrak{g}^{\vee}$ is the Langlands dual of $\mathfrak{g}$. By taking the moment map image of Mirković-Vilonen cycles, Anderson [A] defined a family of convex polytopes in $\mathfrak{h}_{\mathbb{R}}$, which are called Mirković-Vilonen $\left(M V\right.$ for short) polytopes. Here $\mathfrak{h}$ is the Cartan subalgebra of $\mathfrak{g}$ and $\mathfrak{h}_{\mathbb{R}}$ the real form of $\mathfrak{h}$.

Key words and phrases. Crystal basis, PBW basis, MV polytopes.

Mathematics Subject Classification (2010): Primary 17B37; Secondary 17B67, 81R10, 81R50.

Reseach of the author is supported by Grant-in-Aid for Scientific Resaerch (C) 20540009, Japan Society for the Promontion Science. 
After these works, Kamnitzer [Kam1], Kam2] gave a combinatorial characterization of MV polytopes by using a notion of Berenstein-Zelevinsky (BZ for short) data. A BZ datum is a certain family of integers (see Section 5 for details), which is introduced by Berenstein, Fomin and Zelevinsky [BFZ]. Moreover he showed that the set of MV polytopes has a crystal structure which is isomorphic to $B(\infty)$. That is, he constructed

(e) a realization of $B(\infty)$ in terms of MV polytopes.

We remark that he also proved that the above crystal structure on the set of MV polytopes coincides with the crystal structure coming from one on the set of Mirković-Vilonen cycles, which is introduced in $[\mathrm{BG}$.

1.3. In the current paper, we focus on the case $\mathfrak{g}=\mathfrak{s} l_{n+1}(\mathbb{C})$. The aim of the paper is to give explicit isomorphisms between the three realizations (c), (d) and (e) of $B(\infty)$. In a process for constructing these isomorphisms, we can also give a quiver theoretical description of MV polytopes (or BZ data) in type $A_{n}$.

As a by-product, we give a new proof of the Anderson-Mirković (AM for short) conjecture. The AM conjecture is a conjecture on the explicit forms of the actions of lowering Kashiwara operators on the set of MV polytopes, which is conjectured by Anderson and Mirković (unpublished) and proved by Kamnitzer [Kam2] (see Theorem 5.5.1).

1.4. This paper is organized as follows. In Section 2, we give a quick review on the theory of crystals. After recalling basic properties of PBW basis of the negative half of quantum enveloping algebras in Section 3, we introduce a crystal structure on PBW basis in Section 4 (see Theorem 4.2.1). We remark that this is just a reformulation of the result of Reineke [Re. In Section 5, after reviewing some of basic facts on MV polytopes following Kamnitzer [Kam1], Kam2], we construct an isomorphism from a parametrizing set of PBW basis (so-called Lusztig data) to the set of MV polytopes in explicit way (see Theorem 5.6.3 which will be proved in Section 7). In other words, this isomorphism tells us an explicit relation between the realization (d) of $B(\infty)$ and (e). In Section 6, we give a quiver theoretical interpretation of a $\mathrm{BZ}$ datum in type $A$ (see Corollary 6.3.3). In this consideration, the work of Berenstein, Fomin and Zelevinsky BFZ plays an important role. In Section 7, as we mentioned above, we give a proof of Theorem 5.6.3. In the first half of this section, we give a short review on a Lagrangian construction of $B(\infty)$, following Kashiwara and the author [KS]. This is just the realization (c). Since the explicit isomorphism between the realization (c) and (d) is already known, the problem can be translated as follows: "prove that the induced map form the realization (c) to (e) is an isomorphism of crystals". In the second half, we prove this problem by using the results of Section 6 and quiver theoretical considerations. Finally, in Section 8, we give a new proof of the AM conjecture in type $A$, as an application of the previous results.

1.5. Very recently, another quiver theoretical interpretation of BZ data was given by Baumann, Kamnitzer and Sadanand ([KamS for type $A$, and $[\mathrm{BK}$ for type $A, D, E$ ). They gave similar results as our article (for example, see Theorem 21 in [BK]). But their approach is different from ours. Indeed, in their interpretation, they use the representation theory of preprojective algebras (in other words, the double quiver of Dynkin type with certain relations). On the other hand, in our construction, we only use the ordinary Dynkin quiver. In addition to that, as we already mentioned above, we focus only on type $A$. By this restriction, we can get an explicit formula for computing each BZ datum in terms of the realization (d) of $B(\infty)$ in type $A$. Consequently, we also have a new proof of the AM conjecture in type $A$. Moreover, our approach can be generalized in affine type $A$ ( $c f$. [NSS1, [NSS2]). In other words, this article is the first step for the above generalization. 
Acknowledgment. The author is grateful to Professor Saburo Kakei, Professor Yoshiyuki Kimura, Professor Satoshi Naito, Professor Daisuke Sagaki and Professor Yoshihiro Takeyama for valuable discussions. The author also would like to thank to Professor Pierre Baumann and Professor Joel Kamnitzer for valuable comments on the earlier draft.

\section{Preliminaries}

2.1. Notations. In this article, we assume $\mathfrak{g}=\mathfrak{s} l_{n+1}(\mathbb{C})$. Let $\mathfrak{h}$ be the Cartan subalgebra of $\mathfrak{g}$. We denote by $\alpha_{i} \in \mathfrak{h}^{*}(i \in I=\{1,2, \cdots, n\})$ the simple roots of $\mathfrak{g}$, and $h_{i} \in \mathfrak{h}(i \in I)$ the simple coroot of $\mathfrak{g}$; note that $\left\langle h_{i}, \alpha_{j}\right\rangle=a_{i j}$ for $i, j \in I$, where $\langle\cdot, \cdot\rangle$ denotes the canonical pairing between $\mathfrak{h}$ and $\mathfrak{h}^{*},\left(a_{i j}\right)_{i, j \in I}$ the Cartan matrix of type $A_{n}$. We denote by $P, Q$ and $\Delta^{+}$the weight lattice, the root lattice and the set of all positive roots, respectively. Let $W=\mathfrak{S}_{n+1}$ be the Weyl group of $\mathfrak{g}$. It is generated by simple reflections $s_{i}=s_{\alpha_{i}}(i \in I)$. Let $e$ and $w_{0}$ be the unit element and the longest element of $W$, respectively.

Let $U_{q}=U_{q}(\mathfrak{g})$ be the quantized universal enveloping algebra of type $A_{n}$ with generators $e_{i}, f_{i}, t_{i}^{ \pm}(i \in I)$. It is an associative algebra over $\mathbb{Q}(q)$. Let $U_{q}^{-}$be the subalgebra of $U_{q}$ generated by $f_{i}(i \in I)$. Define $[l]=\frac{q^{l}-q^{-l}}{q-q^{-1}}$ and $[k] !=\prod_{l=1}^{k}[l]$. For $x \in U_{q}(\mathfrak{g})$, we denote $x^{(k)}=x^{k} /[k] !$.

\subsection{Crystals.}

Definition 2.2.1. (1) Consider the following data:

(i) a set $B$,

(ii) a map wt : $B \rightarrow P$,

(iii) $\operatorname{maps} \varepsilon_{i}: B \rightarrow \mathbb{Z} \sqcup\{-\infty\}, \varphi_{i}: B \rightarrow \mathbb{Z} \sqcup\{-\infty\}(i \in I)$,

(iv) maps $\widetilde{e}_{i}: B \rightarrow B \sqcup\{0\}, \widetilde{f}_{i}: B \rightarrow B \sqcup\{0\}(i \in I)$.

The sixtuple $\left(B ; \mathrm{wt}, \varepsilon_{i}, \varphi_{i}, \widetilde{e}_{i}, \widetilde{f}_{i}\right)$ (denoted by $B$, for short) is called a crystal if it satisfies the following axioms:

(C1) $\varphi_{i}(b)=\varepsilon_{i}(b)+\left\langle h_{i}, \mathrm{wt}(b)\right\rangle$.

(C2) If $b \in B$ and $\widetilde{e}_{i} b \in B$, then $\operatorname{wt}\left(\widetilde{e}_{i} b\right)=\operatorname{wt}(b)+\alpha_{i}, \varepsilon_{i}\left(\widetilde{e}_{i} b\right)=\varepsilon_{i}(b)-1, \varphi_{i}\left(\widetilde{e}_{i} b\right)=$ $\varphi_{i}(b)+1$.

$\left(\mathrm{C} 2{ }^{\prime}\right)$ If $b \in B$ and $\widetilde{f}_{i} b \in B$, then $\operatorname{wt}\left(\widetilde{f}_{i} b\right)=\operatorname{wt}(b)-\alpha_{i}, \varepsilon_{i}\left(\widetilde{f}_{i} b\right)=\varepsilon_{i}(b)+1, \varphi_{i}\left(\widetilde{f}_{i} b\right)=$ $\varphi_{i}(b)-1$.

(C3) For $b, b^{\prime} \in B, b^{\prime}=\widetilde{e}_{i} b$ if and only if $b=\widetilde{f}_{i} b^{\prime}$.

(C4) For $b \in B$, if $\varphi_{i}(b)=-\infty$, then $\widetilde{e}_{i} b=\widetilde{f}_{i} b=0$.

(2) For two crystals $B_{1}$ and $B_{2}$, a morphism $\psi$ from $B_{1}$ to $B_{2}$ is a map $B_{1} \sqcup\{0\} \rightarrow B_{2} \sqcup\{0\}$ that satisfies the following conditions:

(i) $\psi(0)=0$.

(ii) If $b \in B_{1}$ and $\psi(b) \in B_{2}$, then $\operatorname{wt}(\psi(b))=\operatorname{wt}(b), \varepsilon_{i}(\psi(b))=\varepsilon_{i}(b)$ and $\varphi_{i}(\psi(b))=$ $\varphi_{i}(b)$.

(iii) If $b, b^{\prime} \in B_{1}$ satisfy $b^{\prime}=\widetilde{f}_{i}(b)$ and $\varphi(b), \varphi\left(b^{\prime}\right) \in B_{2}$, then $\psi\left(b^{\prime}\right)=\widetilde{f}_{i}(\psi(b))$.

$A$ morphism $\psi: B_{1} \rightarrow B_{2}$ is called an isomorphism, if $\psi$ induces an bijective map $B_{1} \sqcup$ $\{0\} \rightarrow B_{2} \sqcup\{0\}$ and it commutes with all $\widetilde{e}_{i}$ and $\widetilde{f}_{i}$.

2.3. Crystal basis of $U_{q}^{-}$. We shall recall the definition of the crystal basis of $U_{q}^{-}$. Let $e_{i}^{\prime}$ and $e_{i}^{\prime \prime}$ be endomorphisms of $U_{q}^{-}$defined by

$$
\left[e_{i}, x\right]=\frac{t_{i} e_{i}^{\prime \prime}(x)-t_{i}^{-1} e_{i}^{\prime}(x)}{q-q^{-1}} \quad\left(x \in U_{q}^{-}\right) .
$$


It is known that any element $x \in U_{q}^{-}$can be uniquely written as

$$
x=\sum_{k \geq 0} f_{i}^{(k)} x_{k} \quad \text { with } e_{i}^{\prime}\left(x_{k}\right)=0 .
$$

Define modified root operators (so-called Kashiwara operators) $\widetilde{e}_{i}$ and $\widetilde{f}_{i}$ on $U_{q}^{-}$by

$$
\widetilde{e}_{i} x=\sum_{k \geq 1} f_{i}^{(k-1)} x_{k}, \quad \widetilde{f}_{i} x=\sum_{k \geq 0} f_{i}^{(k+1)} x_{k} .
$$

Let $\mathcal{A}$ be the subring of $\mathbb{Q}(q)$ consisting of rational functions without a pole at $q=0$. Set

$$
\begin{gathered}
L(\infty)=\sum_{k \geq 0, i_{1}, \cdots, i_{k} \in I} \mathcal{A} \widetilde{f}_{i_{1}} \cdots \widetilde{f}_{i_{k}} \cdot 1 \subset U_{q}^{-}, \\
B(\infty)=\left\{\widetilde{f}_{i_{1}} \cdots \widetilde{f}_{i_{k}} \cdot 1 \bmod q L(\infty) \mid k \geq 0, i_{1}, \cdots, i_{k} \in I\right\} .
\end{gathered}
$$

Then the following properties hold:

(1) $\widetilde{e}_{i} L(\infty) \subset L(\infty)$ and $\widetilde{f}_{i} L(\infty) \subset L(\infty)$,

(2) $B(\infty)$ is a $\mathbb{Q}$-basis of $L(\infty) / q L(\infty)$,

(3) $\widetilde{e}_{i} B(\infty) \subset B(\infty) \cup\{0\}$ and $\widetilde{f}_{i} B(\infty) \subset B(\infty)$.

We call $(L(\infty), B(\infty))$ the crystal basis of $U_{q}^{-}$.

For $b \in B(\infty)$, we set

$\operatorname{wt}(b)=$ the weight of $b, \quad \varepsilon_{i}(b)=\max \left\{k \geq 0 \mid \widetilde{e}_{i}^{k}(b) \neq 0\right\}, \quad \varphi_{i}(b)=\varepsilon_{i}(b)+\left\langle h_{i}, \operatorname{wt}(b)\right\rangle$.

Then $\left(B(\infty)\right.$, wt, $\left.\varepsilon_{i}, \varphi_{i}, \widetilde{e}_{i}, \widetilde{f}_{i}\right)$ is a crystal in the sense of Definition 2.2.1.

2.4. Orderings on the set of positive roots. Since $\mathfrak{g}=\mathfrak{s} l_{n+1}(\mathbb{C})$, any positive root $\beta \in \Delta^{+}$can be uniquely written as

$$
\beta=\sum_{p=i_{\beta}}^{j_{\beta}-1} \alpha_{p} \quad\left(\text { for some } 1 \leq i_{\beta}<j_{\beta} \leq n+1\right) .
$$

The correspondence $\beta \mapsto\left(i_{\beta}, j_{\beta}\right)$ defines a bijection $\Delta^{+} \stackrel{\sim}{\rightarrow} \Pi$ where

$$
\Pi=\{(i, j) \mid 1 \leq i<j \leq n+1\} .
$$

In the rest of this article, we sometimes identify $\Pi$ with $\Delta^{+}$via the above bijection.

Let $N=n(n+1) / 2$ be the length of $w_{0}$ and fix $w_{0}=s_{i_{1}} s_{i_{2}} \cdots s_{i_{N}}\left(i_{1}, i_{2}, \cdots, i_{N} \in I\right)$ a reduced expression of $w_{0}$. We denote by $\mathbf{i}=\left(i_{1}, i_{2}, \cdots, i_{N}\right)$ the corresponding reduced word. Set $\beta_{k}=s_{i_{1}} s_{i_{2}} \cdots s_{i_{k-1}}\left(\alpha_{i_{k}}\right)(1 \leq k \leq N)$. Then we have $\Pi=\left\{\beta_{1}, \beta_{2}, \cdots, \beta_{N}\right\}$. That is, a reduced word $\mathbf{i}$ defines a bijection $\Upsilon_{\mathbf{i}}:\{1,2, \cdots, N\} \stackrel{\sim}{\rightarrow} \Pi$ and it induces a total ordering $\leq_{\mathrm{i}}$ on $\Pi$;

$$
\left(i_{1}, j_{1}\right) \leq_{\mathbf{i}}\left(i_{2}, j_{2}\right) \stackrel{\text { def }}{\Longleftrightarrow} \Upsilon_{\mathbf{i}}^{-1}\left(i_{1}, j_{1}\right) \leq \Upsilon_{\mathbf{i}}^{-1}\left(i_{2}, j_{2}\right) .
$$

Example 2.4.1. Let $\mathbf{i}_{0}$ be the lexicographically minimal reduced word given by

$$
\mathbf{i}_{0}=(1,2,1,3,2,1, \cdots, n, n-1, \cdots, 1) .
$$

Then we have

$$
\left(i_{1}, j_{1}\right) \leq_{\mathbf{i}_{0}}\left(i_{2}, j_{2}\right) \text { if and only if }\left\{\begin{array}{l}
j_{1}<j_{2} \\
\text { or } \\
j_{1}=j_{2} \text { and } i_{1} \leq i_{2}
\end{array}\right.
$$




\section{PBW BASIS AND LUSZTig DATA ASSOCIATED TO REDUCED WORDS}

3.1. PBW basis of quantized universal enveloping algebras. For $i \in I$, introduce an $\mathbb{Q}(q)$-algebra automorphism $T_{i}$ of $U_{q}$ as :

$$
\begin{gathered}
T_{i}\left(e_{j}\right):= \begin{cases}-f_{i} t_{i} & (i=j), \\
\sum_{k=0}^{-a_{i j}}(-1)^{a_{i j}+k} q^{a_{i j}+k} e_{i}^{(k)} e_{j} e_{i}^{\left(-a_{i j}-k\right)} & (i \neq j),\end{cases} \\
T_{i}\left(f_{j}\right)= \begin{cases}-t_{i}^{-1} e_{i}, & (i=j), \\
\sum_{k=0}^{-a_{i j}}(-1)^{a_{i j}+k} q^{-a_{i j}-k} f_{i}^{\left(-a_{i j}-k\right)} f_{j} f_{i}^{(k)}, & (i \neq j),\end{cases} \\
T_{i}\left(t_{j}\right)=t_{j} t_{i}^{-a_{i j}} .
\end{gathered}
$$

It is known that these operators satisfy the braid relations:

$$
T_{i} T_{j} T_{i}=T_{j} T_{i} T_{j}\left(a_{i j}=-1\right), \quad T_{i} T_{j}=T_{j} T_{i}\left(a_{i j}=0\right) .
$$

For a reduced word $\mathbf{i}=\left(i_{1}, i_{2}, \cdots, i_{N}\right)$ and $\mathbf{c}=\left(c_{1}, \cdots, c_{N}\right) \in \mathbb{Z}_{\geq 0}^{N}$, we define

$$
P_{\mathbf{i}}(\mathbf{c})=f_{i_{1}}^{\left(c_{1}\right)}\left(T_{i_{1}}\left(f_{i_{1}}^{\left(c_{2}\right)}\right)\right) \cdots\left(T_{i_{1}} T_{i_{2}} \cdots T_{i_{N-1}}\left(f_{i_{N}}^{\left(c_{N}\right)}\right)\right)
$$

and

$$
B_{\mathbf{i}}=\left\{P_{\mathbf{i}}(\mathbf{c}) \mid \mathbf{c} \in \mathbb{Z}_{\geq 0}^{N}\right\}, \quad L_{\mathbf{i}}=\sum_{\mathbf{c}} \mathcal{A} P_{\mathbf{i}}(\mathbf{c})
$$

Proposition 3.1.1 ([L1], $[\underline{\mathrm{S}}])$. (1) $B_{\mathbf{i}}$ is a $\mathbb{Q}(q)$-basis of $U_{q}^{-}$.

(2) $L_{\mathbf{i}}=L(\infty)$. Moreover $B_{\mathbf{i}}$ is a free $\mathcal{A}$-basis of $L(\infty)$.

(3) $B_{\mathbf{i}} \equiv B(\infty) \bmod q L(\infty)$.

Definition 3.1.2. For a giving reduced word $\mathbf{i}$, the basis $B_{\mathbf{i}}=\left\{P_{\mathbf{i}}(\mathbf{c}) \mid \mathbf{c} \in \mathbb{Z}_{\geq 0}^{N}\right\}$ is called the $P B W$ basis of $U_{q}^{-}$associated to a reduced word $\mathbf{i}$. There is a bijection $\Xi_{\mathbf{i}}: \mathbb{Z}_{\geq 0}^{N} \stackrel{\sim}{\rightarrow} B(\infty)$ defined by $\mathbf{c} \mapsto P_{\mathbf{i}}(\mathbf{c}) \bmod q L(\infty)$. For $b \in B(\infty)$, we call $\Xi_{\mathbf{i}}^{-1}(b) \in \mathbb{Z}_{\geq 0}^{N}$ the $\mathbf{i}$-Lusztig datum of $b \in B(\infty)$.

3.2. The transition maps. For a reduced word $\mathbf{i}$, consider

$$
\mathcal{B}^{\mathbf{i}}=\left\{\mathbf{a}^{\mathbf{i}}=\left(a_{i, j}^{\mathbf{i}}\right)_{(i, j) \in \Pi} \mid a_{i, j}^{\mathbf{i}} \in \mathbb{Z}_{\geq 0} \text { for any }(i, j) \in \Pi\right\}
$$

the set of all $N$-tuples of non-negative integers indexed by $\Pi$. From now on, we regard $\mathcal{B}^{\mathbf{i}}$ as the set of all $\mathbf{i}$-Lusztig data via the bijection $\mathbb{Z}_{\geq 0}^{N} \stackrel{\sim}{\rightarrow} \mathcal{B}^{\mathbf{i}}$ induced from $\Upsilon_{\mathbf{i}}:\{1, \cdots, N\} \stackrel{\sim}{\rightarrow} \Pi$. For two reduced words $\mathbf{i}$ and $\mathbf{i}^{\prime}$, let us consider the following composition of the bijections:

$$
R_{\mathbf{i}}^{\mathbf{i}^{\prime}}=\Xi_{\mathbf{i}^{\prime}}^{-1} \circ \Xi_{\mathbf{i}}: \mathcal{B}^{\mathbf{i}} \stackrel{\sim}{\rightarrow} B(\infty) \stackrel{\sim}{\rightarrow} \mathcal{B}^{\mathbf{i}^{\prime}}
$$

We call $R_{\mathbf{i}}^{\mathbf{i}^{\prime}}$ the transition map form $\mathbf{i}$ to $\mathbf{i}^{\prime}$. The explicit form of $R_{\mathbf{i}}^{\mathbf{i}^{\prime}}$ is known $([\overline{B F Z}])$, but we omit to give it in this article.

For an arbitrarily reduced word $\mathbf{i}$, the set of all $\mathbf{i}$-Lusztig data $\mathcal{B}^{\mathbf{i}}$ has a crystal structure which is induced form the bijection $\Xi_{\mathbf{i}}: \mathcal{B}^{\mathbf{i}} \stackrel{\sim}{\rightarrow} B(\infty)$. Especially, for the lexicographically minimal reduced word $\mathbf{i}_{0}$, we denote $\mathcal{B}=\mathcal{B}^{\mathbf{i}_{0}}$ and $\mathbf{a}=\mathbf{a}^{\mathbf{i}_{0}} \in \mathcal{B}$. It has a central role in this article. In the next section we will give the crystal structure on $\mathcal{B}=\mathcal{B}^{\mathbf{i}_{0}}$ in explicit way. 
3.3. *-structure. Define a $\mathbb{Q}(q)$-algebra anti-involution $*$ of $U_{q}$ by

$$
e_{i}^{*}=e_{i}, \quad f_{i}^{*}=f_{i}, \quad\left(t_{i}^{ \pm}\right)^{*}=t_{i}^{\mp} \quad(i \in I) .
$$

By the construction, it is easy to see $L(\infty)^{*}=L(\infty)$. Therefore $*$ induces a $\mathbb{Q}$-linear automorphism of $L(\infty) / q L(\infty)$. Moreover the following theorem is known.

Theorem 3.3.1 ([K3]). The anti-involution $*$ induces an involutive bijection on $B(\infty)$.

By the above theorem, we can define the operators $\widetilde{e}_{i}^{*}, \widetilde{f}_{i}^{*}$ on $B(\infty)$ by

$$
\widetilde{e}_{i}^{*}=* \widetilde{e}_{i} *, \quad \widetilde{f}_{i}^{*}=* \widetilde{f}_{i} * .
$$

By the definition, it is obvious that $b_{\infty}^{*}=b_{\infty}$. Here $b_{\infty}$ is the highest weight element of $B(\infty)$. Therefore we have

$$
\varepsilon_{i}\left(b^{*}\right)=\max \left\{k \geq 0 \mid\left(\widetilde{e}_{i}^{*}\right)^{k}(b) \neq 0\right\}
$$

for $b \in B(\infty)$. From now on, we denote $\varepsilon_{i}^{*}(b)=\varepsilon_{i}\left(b^{*}\right)$.

Define a $\mathbb{Q}(q)$-algebra automorphism $T_{i}^{*}(i \in I)$ by

$$
T_{i}^{*}=* \circ T_{i} \circ *
$$

Then we have

$$
\begin{aligned}
P_{\mathbf{i}}^{*}(\mathbf{c}) & =\left(f_{i_{1}}^{\left(c_{1}\right)}\left(T_{i_{1}}\left(f_{i_{1}}^{\left(c_{2}\right)}\right)\right) \cdots\left(T_{i_{1}} T_{i_{2}} \cdots T_{i_{N-1}}\left(f_{i_{N}}^{\left(c_{N}\right)}\right)\right)\right)^{*} \\
& =\left(T_{i_{1}}^{*} T_{i_{2}}^{*} \cdots T_{i_{N-1}}^{*}\left(f_{i_{N}}^{\left(c_{N}\right)}\right)\right) \cdots\left(T_{i_{1}}^{*}\left(f_{i_{1}}^{\left(c_{2}\right)}\right)\right) f_{i_{1}} .
\end{aligned}
$$

Set

$$
B_{\mathbf{i}}^{*}=\left\{P_{\mathbf{i}}^{*}(\mathbf{c}) \mid \mathbf{c} \in \mathbb{Z}_{\geq 0}^{N}\right\} .
$$

It also gives a $\mathbb{Q}(q)$-basis of $U_{q}^{-}$. We call it the $*-P B W$ basis of $U_{q}^{-}$associated to a reduced word $\mathbf{i}$.

By Theorem 3.3.1, we have the following corollary.

Corollary 3.3.2.

$$
\left\{P_{\mathbf{i}}^{*}(\mathbf{c}) \bmod q L(\infty) \mid \mathbf{c} \in \mathbb{Z}_{\geq 0}^{N}\right\}=B(\infty) .
$$

\section{Crystal structure on $\mathbf{i}_{0}$-Lusztig data}

4.1. Definition of crystal structures. We shall define two crystal structures on the set of all $\mathbf{i}_{0}$-Lusztig datum $\mathcal{B}$. For $\mathbf{a} \in \mathcal{B}$, define the weight wt(a) of $\mathbf{a}$ by

$$
\mathrm{wt}(\mathbf{a})=-\sum_{i \in I} m_{i} \alpha_{i}, \quad \text { where } \quad m_{i}=\sum_{k=1}^{i} \sum_{l=i+1}^{n+1} a_{k, l} \quad(i \in I) .
$$

For $i \in I$, set

$$
\begin{gathered}
A_{k}^{(i)}(\mathbf{a})=\sum_{s=1}^{k}\left(a_{s, i+1}-a_{s-1, i}\right) \quad(1 \leq k \leq i), \\
A_{l}^{*(i)}(\mathbf{a})=\sum_{t=l+1}^{n+1}\left(a_{i, t}-a_{i+1, t+1}\right) \quad(i \leq l \leq n) .
\end{gathered}
$$

Here we set $a_{0, i}=0$ and $a_{i+1, n+2}=0$. Define

$$
\varepsilon_{i}(\mathbf{a})=\max \left\{A_{1}^{(i)}(\mathbf{a}), \cdots, A_{i}^{(i)}(\mathbf{a})\right\}, \quad \varphi_{i}(\mathbf{a})=\varepsilon_{i}(\mathbf{a})+\left\langle h_{i}, \operatorname{wt}(\mathbf{a})\right\rangle,
$$




$$
\varepsilon_{i}^{*}(\mathbf{a})=\max \left\{A_{i}^{*(i)}(\mathbf{a}), \cdots, A_{n}^{*(i)}(\mathbf{a})\right\}, \quad \varphi_{i}^{*}(\mathbf{a})=\varepsilon_{i}^{*}(\mathbf{a})+\left\langle h_{i}, \operatorname{wt}(\mathbf{a})\right\rangle .
$$

Let

$$
\begin{aligned}
& k_{e}=\min \left\{1 \leq k \leq i \mid \varepsilon_{i}(\mathbf{a})=A_{k}^{(i)}(\mathbf{a})\right\}, \quad k_{f}=\max \left\{1 \leq k \leq i \mid \varepsilon_{i}(\mathbf{a})=A_{k}^{(i)}(\mathbf{a})\right\}, \\
& l_{e}=\max \left\{i \leq l \leq n \mid \varepsilon_{i}^{*}(\mathbf{a})=A_{l}^{*(i)}(\mathbf{a})\right\}, \quad l_{f}=\min \left\{i \leq l \leq n \mid \varepsilon_{i}^{*}(\mathbf{a})=A_{l}^{*(i)}(\mathbf{a})\right\} .
\end{aligned}
$$

For a given $\mathbf{a} \in \mathcal{B}$, we introduce four $N$-tuples of integers $\mathbf{a}^{(p)}=\left(a_{k, l}^{(p)}\right)(p=1,2,3,4)$ by

$$
\begin{aligned}
& a_{k, l}^{(1)}= \begin{cases}a_{k_{e}, i}+1 & \left(k=k_{e}, l=i\right), \\
a_{k_{e}, i+1}-1 & \left(k=k_{e}, l=i+1\right), \\
a_{k, l} & (\text { otherwise })\end{cases} \\
& a_{k, l}^{(2)}= \begin{cases}a_{k_{f}, i}-1 & \left(k=k_{f}, l=i\right), \\
a_{k_{f}, i+1}+1 & \left(k=k_{f}, l=i+1\right), \\
a_{k, l} & (\text { otherwise }),\end{cases} \\
& a_{k, l}^{(3)}= \begin{cases}a_{i, l_{e}+1}-1 & \left(k=i, l=l_{e}+1\right), \\
a_{i+1, l_{e}+1}+1 & \left(k=i+1, l=l_{e}+1\right), \\
a_{k, l} & (\text { otherwise }) .\end{cases} \\
& a_{k, l}^{(4)}= \begin{cases}a_{i, l_{f}+1}+1 & \left(k=i, l=l_{f}+1\right), \\
a_{i+1, l_{f}+1}-1 & \left(k=i+1, l=l_{f}+1\right), \\
a_{k, l} & (\text { otherwise }) .\end{cases}
\end{aligned}
$$

Lemma 4.1.1. (1) For any $\mathbf{a} \in \mathcal{B}$ with $\varepsilon_{i}(\mathbf{a})>0$, $\mathbf{a}^{(1)}$ is an $N$-tuple of non-negative integers. In other words, $\mathbf{a}^{(1)}$ is an element of $\mathcal{B}$.

(2) For any $\mathbf{a} \in \mathcal{B}$ with $\varepsilon_{i}^{*}(\mathbf{a})>0, \mathbf{a}^{(3)}$ is an element of $\mathcal{B}$.

(3) For any $\mathbf{a} \in \mathcal{B}$, both $\mathbf{a}^{(2)}$ and $\mathbf{a}^{(4)}$ are elements of $\mathcal{B}$.

Proof. We only give a proof of (1). It is enough to show that $a_{k_{e}, i+1}>0$. If $k_{e}=1$, we have $a_{k_{e}, i+1}=A_{1}^{(i)}(\mathbf{a})=\varepsilon_{i}(\mathbf{a})>0$. Assume $k_{e}>1$. Then, by the definition of $k_{e}$, we have $A_{k_{e}-1}^{(i)}(\mathbf{a})<A_{k_{e}}^{(i)}(\mathbf{a})$. Therefore $A_{k_{e}}^{(i)}(\mathbf{a})-A_{k_{e}-1}^{(i)}(\mathbf{a})=a_{k_{e}, i+1}-a_{k_{e}-1, i}>0$. Since $a_{k_{e}-1, i} \geq 0$, we have $a_{k_{e}, i+1}>0$.

Now we define Kashiwara operators on $\mathcal{B}$ as:

$$
\begin{aligned}
& \widetilde{e}_{i} \mathbf{a}= \begin{cases}0 & \left(\text { if } \varepsilon_{i}(\mathbf{a})=0\right), \\
\mathbf{a}^{(1)} & \left(\text { if } \varepsilon_{i}(\mathbf{a})>0\right),\end{cases} \\
& \widetilde{e}_{i}^{*} \mathbf{a}= \begin{cases}0 & \left(\text { if } \varepsilon_{i}^{*}(\mathbf{a})=0\right), \\
\mathbf{a}^{(3)} & \left(\text { if } \varepsilon_{i}^{*}(\mathbf{a})>0\right),\end{cases}
\end{aligned}
$$

Proposition 4.1.2. (1) (B, wt, $\left.\varepsilon_{i}, \varphi_{i}, \widetilde{e}_{i}, \widetilde{f}_{i}\right)$ is a crystal in the sense of Definition 2.2.1. (2) $\left(\mathcal{B}, \mathrm{wt}, \varepsilon_{i}^{*}, \varphi_{i}^{*}, \widetilde{e}_{i}^{*}, \widetilde{f}_{i}^{*}\right)$ is a crystal in the sense of Definition 2.2.1.

From the definition, one can easily check the axiom $(\mathrm{C} 1) \sim(\mathrm{C} 4)$. So we omit to give a detail.

4.2. Crystal structure on PBW basis associated to $\mathbf{i}_{0}$. As we mentioned in the previous subsection, we regard $\mathcal{B}$ as the set of $\mathbf{i}_{0}$-Lusztig datum and denote by $\left\{P_{\mathbf{i}_{0}}(\mathbf{a}) \mid \mathbf{a} \in\right.$ $\mathcal{B}\}$ the corresponding PBW basis. 
Theorem 4.2.1. (1) We have

$$
\widetilde{e}_{i} P_{\mathbf{i}_{0}}(\mathbf{a}) \equiv P_{\mathbf{i}_{0}}\left(\widetilde{e}_{i} \mathbf{a}\right) \bmod q L(\infty) \quad \text { and } \quad \widetilde{f}_{i} P_{\mathbf{i}_{0}}(\mathbf{a}) \equiv P_{\mathbf{i}_{0}}\left(\widetilde{f}_{i} \mathbf{a}\right) \bmod q L(\infty)
$$

(2) We have

$$
\widetilde{e}_{i} P_{\mathbf{i}_{0}}^{*}(\mathbf{a}) \equiv P_{\mathbf{i}_{0}}^{*}\left(\widetilde{e}_{i}^{*} \mathbf{a}\right) \bmod q L(\infty) \quad \text { and } \quad \widetilde{f}_{i} P_{\mathbf{i}_{0}}^{*}(\mathbf{a}) \equiv P_{\mathbf{i}_{0}}^{*}\left(\widetilde{f}_{i}^{*} \mathbf{a}\right) \bmod q L(\infty) .
$$

Remark . The formulas (2) is proved by Reineke [Re] (see also [Sav], EK]). Note that in $\left[\mathrm{Re}\right.$, he denotes our $\widetilde{e}_{i}^{*}$ and $\widetilde{f}_{i}^{*}$ on $\mathcal{B}$, by $\widetilde{e}_{i}$ and $\widetilde{f}_{i}$, respectively. The formulas (1) is proved by the similar method.

By the definition, we immediately have the next corollary.

Corollary 4.2.2. (1) We have

$$
\widetilde{e}_{i}^{*} P_{\mathbf{i}_{0}}(\mathbf{a}) \equiv P_{\mathbf{i}_{0}}\left(\widetilde{e}_{i}^{*} \mathbf{a}\right) \bmod q L(\infty) \quad \text { and } \quad \widetilde{f}_{i}^{*} P_{\mathbf{i}_{0}}(\mathbf{a}) \equiv P_{\mathbf{i}_{0}}\left(\widetilde{f}_{i}^{*} \mathbf{a}\right) \bmod q L(\infty) .
$$

(2) As a by-product, we have that $\mathcal{B}$ is isomorphic to $B(\infty)$ as a crystal with $*$-structure.

\section{Mirković-Vilonen polytopes in type $A_{n}$}

5.1. Mirković-Vilonen polytopes and Berenstein-Zelevinsky data. Let $\Lambda_{i}(i \in I)$ be a fundamental weight for $\mathfrak{g}$. Set

$$
\Gamma_{n}=\bigcup_{i \in I} W \Lambda_{i}
$$

and an element $\gamma \in \Gamma_{n}$ is called a chamber weight. Let $\mathbf{M}=\left(M_{\gamma}\right)_{\gamma \in \Gamma_{n}}$ be a collection of integers indexed by $\Gamma_{n}$. For each $\gamma \in \Gamma_{n}$, we call $M_{\gamma}$ the the $\gamma$-component $\mathbf{M}$, and denote it by $(\mathbf{M})_{\gamma}$.

For a given $\mathbf{M}=\left(M_{\gamma}\right)_{\gamma \in \Gamma_{n}}$, consider the following polytope in $\mathfrak{h}_{\mathbb{R}}$ :

$$
P(\mathbf{M})=\left\{h \in \mathfrak{h}_{\mathbb{R}} \mid\langle h, \gamma\rangle \geq M_{\gamma}\left(\forall \gamma \in \Gamma_{n}\right)\right\} .
$$

Definition 5.1.1. (1) A polytope $P(\mathbf{M})$ is called a pseudo-Weyl polytope if it satisfies the following condition:

(BZ-1) (edge inequalities) for all $w \in W$ and $i \in I$,

$$
M_{w \Lambda_{i}}+M_{w s_{i} \Lambda_{i}}+\sum_{j \in I \backslash\{i\}} a_{j i} M_{w \Lambda_{j}} \leq 0
$$

(2) A pseudo-Weyl polytope $P(\mathbf{M})$ is called a Mirković-Vilonen ( $M V$ for short) polytope if it satisfies the following condition:

(BZ-2) (3-term relations) for every $w \in W$ and $i, j \in I$ with $a_{i j}=a_{j i}=-1$ and $w s_{i}>w$, $w s_{j}>w$,

$$
M_{w s_{i} \Lambda_{i}}+M_{w s_{j} \Lambda_{j}}=\min \left\{M_{w \Lambda_{i}}+M_{w s_{i} s_{j} \Lambda_{j}}, M_{w \Lambda_{j}}+M_{w s_{j} s_{i} \Lambda_{i}}\right\} .
$$

Here $\left(a_{i j}\right)_{i, j \in I}$ is the Cartan matrix of type $A_{n}$ and $>$ is the strong Bruhat ordering of $W$. If $P(\mathbf{M})$ is a $M V$ polytope, the corresponding collection of integers $\mathbf{M}=\left(M_{\gamma}\right)_{\gamma \in \Gamma_{n}}$ is called Berenstein-Zelevinsky (BZ for short) datum of type $A_{n}$.

Remark . For a collection of integers $\mathbf{M}=\left(M_{\gamma}\right)_{\gamma \in \Gamma_{n}}$ which satisfies the condition (BZ-1), set

$$
\mu_{w}=\sum_{i \in I} M_{w \Lambda_{i}} w h_{i} \in \mathfrak{h}_{\mathbb{R}} \quad(w \in W)
$$

and consider a collection of vectors

$$
\mu_{\bullet}=\left(\mu_{w}\right)_{w \in W} \subset \mathfrak{h}_{\mathbb{R}} .
$$


Then Kamnitzer [Kam2] showed the corresponding pseudo-Weyl polytope $P(\mathbf{M})$ is the convex hull of $\mu_{\bullet}$. That is, there is a one to one correspondence between the set of pseudoWeyl polytopes and the set of collections of integers which satisfies the condition (BZ-1).

Definition 5.1.2. A BZ datum $\mathbf{M}^{w_{0}}=\left(M_{\gamma}^{w_{0}}\right)_{\gamma \in \Gamma_{n}}$ is called a $w_{0}$-BZ datum of type $A_{n}$ if it satisfies

(BZ-0) (wo-normalization condition) for all $i \in I$,

$$
M_{w_{0} \Lambda_{i}}^{w_{0}}=0
$$

We denote by $\mathcal{B Z}^{w_{0}}$ the set of all $w_{0}-B Z$ data.

A set of integers $K \subset[1, n+1]$ will be called a Maya diagram of rank $n$. We denote by $\mathcal{M}_{n}$ the set of all Maya diagram of rank $n$. Set $\mathcal{M}_{n}^{\times}=\mathcal{M}_{n} \backslash\{\phi,[1, n+1]\}$. From now on, we identify the set of chamber weights $\Gamma_{n}$ with $\mathcal{M}_{n}^{\times}$by the following way: recall that there is a natural action of $W \cong \mathfrak{S}_{n+1}$ on the set $\{1,2, \cdots, n+1\}$. Consider the map $\Gamma_{n} \rightarrow \mathcal{M}_{n}^{\times}$defined by $\gamma=w \Lambda_{i} \mapsto w \cdot[1, i]$. Since this map is bijective, we can identify $\Gamma_{n}$ with $\mathcal{M}_{n}^{\times}$. By the above identification, $\Lambda_{i}$ and $w_{0} \Lambda_{i}$ are regarded as

$$
\Lambda_{i} \leftrightarrow[1, i], \quad w_{0} \Lambda_{i} \leftrightarrow[n-i+2, n+1] \quad(i \in I) .
$$

Under the above identification, the definition of $w_{0}$-BZ datum can be rewritten as follows:

Lemma 5.1.3. A collection $\mathbf{M}^{w_{0}}=\left(M_{K}^{w_{0}}\right)_{K \in \mathcal{M}_{n}^{\times}}$of integers is a $w_{0}-B Z$ datum of type $A_{n}$ if and only if it satisfies the following conditions:

(BZ-0)' for all $i \in I$,

$$
M_{[n-i+2, n+1]}^{w_{0}}=0
$$

(BZ-1)' for every two indices $i \neq j$ in $[1, n+1]$ and every $K \in \mathcal{M}_{n}$ with $K \cap\{i, j\}=\phi$,

$$
M_{K i}^{w_{0}}+M_{K j}^{w_{0}} \leq M_{K i j}^{w_{0}}+M_{K}^{w_{0}}
$$

(BZ-2)' for every three indices $i<j<k$ in $[1, n+1]$ and every $K \in \mathcal{M}_{n}$ with $K \cap\{i, j, k\}=$ $\phi$,

$$
M_{K i k}^{w_{0}}+M_{M j}^{w_{0}}=\min \left\{M_{K i j}^{w_{0}}+M_{K k}^{w_{0}}, M_{K j k}^{w_{0}}+M_{K i}^{w_{0}}\right\} .
$$

Here we denote $M_{K i}^{w_{0}}=M_{K \cup\{i\}}^{w_{0}}$, etc., and set $M_{\phi}^{w_{0}}=M_{[1, n+1]}^{w_{0}}=0$.

Remark . The conditions (BZ-2)' are just the conditions which are called the 3-term relations in [BFZ].

\section{2. e-BZ datum.}

Definition 5.2.1. A collection $\mathbf{M}^{e}=\left(M_{K}^{e}\right)_{K \in \mathcal{M}_{n}^{\times}}$of integers is called a e-normalized Berenstein-Zelevinsky (e-BZ for short) datum of type $A_{n}$ if it satisfies the above (BZ-1)', (BZ-2)' and

(BZ-0)" (e-normalization condition) for all $i \in I$,

$$
M_{[1, i]}^{e}=0 .
$$

We denote by $\mathcal{B Z}^{e}$ the set of all e-BZ data.

For $K \in \mathcal{M}_{n}^{\times}$, let $K^{c}=[1, n+1] \backslash K$ be the complement of $K$ in $[1, n+1]$. For $\mathbf{M}^{w_{0}}=\left(M_{K}^{w_{0}}\right)_{K \in \mathcal{M}_{n}^{\times}} \in \mathcal{B Z}^{w_{0}}$, we define a new collection of integers $\mathbf{M}^{w_{0} *}=\left(M_{K}^{w_{0} *}\right)_{K \in \mathcal{M}_{n}^{\times}}$ by

$$
M_{K}^{w_{0 *}}=M_{K^{c}}^{w_{0}}\left(K \in \mathcal{M}_{n}^{\times}\right)
$$


Lemma 5.2.2. The map $*: \mathbf{M}^{w_{0}} \mapsto \mathbf{M}^{w_{0} *}$ defines a bijection form $\mathcal{B Z}^{w_{0}}$ to $\mathcal{B Z}^{e}$. The inverse $\mathcal{B Z}^{e} \rightarrow \mathcal{B Z}^{w_{0}}$ of the map $*$ is given by

$$
\mathbf{M}^{e}=\left(M_{K}^{e}\right) \mapsto \mathbf{M}^{e *}=\left(M_{K}^{e *}\right), \quad \text { where } M_{K}^{e *}=M_{K^{c}}^{e}\left(K \in \mathcal{M}_{n}^{\times}\right) .
$$

Proof. Let $\mathbf{M}^{w_{0}}=\left(M_{K}^{w_{0}}\right) \in \mathcal{B Z}_{w_{0}}$. Then it is clear that the collection of integers $\mathbf{M}^{w_{0} *}=$ $\left(M_{K}^{\left.w_{0}\right) *}\right)$ satisfies (BZ-0)". Let us prove that $\mathbf{M}^{w_{0} *}$ satisfies (BZ-1)'. Let $i \neq j$ be two indices in $[1, n+1]$ and $K \in \mathcal{M}_{n}$ with $K \cap\{i, j\}=\phi$. For such $i, j$ and $K$, we set $L=K^{c} \backslash\{i, j\}$. Then we have $L \in \mathcal{M}_{n}$ and $L \cap\{i, j\}=\phi$. Since $\mathbf{M}^{w_{0}}$ satisfies (BZ-1)', we have

$$
M_{L i}^{w_{0}}+M_{L j}^{w_{0}} \leq M_{L i j}^{w_{0}}+M_{L}^{w_{0}}
$$

Because

$$
K^{c}=L i j, \quad(K i)^{c}=L j, \quad(K j)^{c}=L i, \quad(K i j)^{c}=L
$$

we have

$$
M_{K}^{w_{0 *}}=M_{L i j}^{w_{0}}, \quad M_{K i}^{w_{0} *}=M_{L j}^{w_{0}}, \quad M_{K j}^{w_{0} *}=M_{L i}^{w_{0}}, \quad M_{K i j}^{w_{0} *}=M_{L}^{w_{0}} .
$$

Therefore we have

$$
M_{K j}^{w_{0} *}+M_{K i}^{w_{0} *} \leq M_{K}^{w_{0} *}+M_{K i j}^{w_{0} *}
$$

This is nothing but (BZ-1)' for $\mathbf{M}^{w_{0} *}$.

By the similar argument we can check (BZ-2)' for $\mathbf{M}^{w_{0} *}$. Thus, $\mathbf{M}^{w_{0} *}$ is an $e-\mathrm{BZ}$ datum. The other statements are clear by the construction.

5.3. Crystal structure on $w_{0}-\mathbf{B Z}$ data. We denote $\mathcal{M V}=\left\{P\left(\mathbf{M}^{w_{0}}\right) \mid \mathbf{M}^{w_{0}} \in \mathcal{B Z}^{w_{0}}\right\}$. In [Kam2, Kamnitzer defines a crystal structure on $\mathcal{M V}$ and shows it is isomorphic to $B(\infty)$ as a crystal. Since the map $\mathcal{B Z}^{w_{0}} \rightarrow \mathcal{M V}$ defined by $\mathbf{M}^{w_{0}} \mapsto P\left(\mathbf{M}^{w_{0}}\right)$ is bijective, we can define a crystal structure on $\mathcal{B Z}^{w_{0}}$ in such a way that the above bijection gives an isomorphism of crystals. In the following, we recall the description of this crystal structure on $\mathcal{B Z}^{w_{0}}$ form Kam2.

Remark . In [Kam2], he uses the set of chamber weights $\Gamma_{n}$ as the index set of $\mathcal{B Z}^{w_{0}}$. But, for later use, we will reformulate the above crystal structure on $\mathcal{B Z}^{w_{0}}$ by using the set of Maya diagrams $\mathcal{M}_{n}^{\times}$instead of $\Gamma_{n}$.

Let $\mathbf{M}^{w_{0}}=\left(M_{K}^{w_{0}}\right) \in \mathcal{B} \mathcal{Z}^{w_{0}}$. Define the weight $\operatorname{wt}\left(\mathbf{M}^{w_{0}}\right)$ of $\mathbf{M}^{w_{0}}$ by

$$
\operatorname{wt}\left(\mathbf{M}^{w_{0}}\right)=\sum_{i \in I} M_{[1, i]}^{w_{0}} \alpha_{i} .
$$

For $i \in I$, we set

$$
\begin{aligned}
& \varepsilon_{i}\left(\mathbf{M}^{w_{0}}\right)=-\left(M_{[1, i]}^{w_{0}}+M_{[1, i+1] \backslash\{i\}}^{w_{0}}-M_{[1, i+1]}^{w_{0}}-M_{[1, i] \backslash\{i\}}^{w_{0}}\right), \\
& \varphi_{i}\left(\mathbf{M}^{w_{0}}\right)=\varepsilon_{i}\left(\mathbf{M}^{w_{0}}\right)+\left\langle h_{i}, \operatorname{wt}\left(\mathbf{M}^{w_{0}}\right)\right\rangle .
\end{aligned}
$$

We remark that $\varepsilon_{i}\left(\mathbf{M}^{w_{0}}\right)$ is a non-negative integer in view of (BZ-1)'.

Let us define the action of Kashiwara operators $\widetilde{e}_{i}$ and $\widetilde{f}_{i}(i \in I)$. We recall the following fact due to Kamnitzer:

Proposition 5.3.1 ([Kam2] $)$. Let $\mathbf{M}^{w_{0}}=\left(M_{K}^{w_{0}}\right) \in \mathcal{B Z}^{w_{0}}$ be a $w_{0}-B Z$ datum.

(1) If $\varepsilon_{i}\left(\mathbf{M}^{w_{0}}\right)>0$, there exists a unique $w_{0}-B Z$ datum which is denoted by $\widetilde{e}_{i} \mathbf{M}^{w_{0}}$ such that

(i) $\left(\widetilde{e}_{i} \mathbf{M}^{w_{0}}\right)_{[1, i]}=M_{[1, i]}^{w_{0}}+1$,

(ii) $\left(\widetilde{e}_{i} \mathbf{M}^{w_{0}}\right)_{K}=M_{K}^{w_{0}}$ for all $K \in \mathcal{M}_{n}^{\times} \backslash \mathcal{M}_{n}^{\times}(i)$. 
Here $\mathcal{M}_{n}^{\times}(i)=\left\{K \in \mathcal{M}_{n}^{\times} \mid i \in K\right.$ and $\left.i+1 \notin K\right\} \subset \mathcal{M}_{n}^{\times}$.

(2) There exists a unique a unique $w_{0}-B Z$ datum which is denoted by $\widetilde{f}_{i} \mathbf{M}^{w_{0}}$ such that

(iii) $\left(\widetilde{f}_{i} \mathbf{M}^{w_{0}}\right)_{[1, i]}=M_{[1, i]}^{w_{0}}-1$,

(iv) $\left(\widetilde{f}_{i} \mathbf{M}^{w_{0}}\right)_{K}=M_{K}^{w_{0}}$ for all $K \in \mathcal{M}_{n}^{\times} \backslash \mathcal{M}_{n}^{\times}(i)$.

If $\varepsilon_{i}\left(\mathbf{M}^{w_{0}}\right)=0$, we set $\widetilde{e}_{i} \mathbf{M}^{w_{0}}=0$.

Theorem 5.3.2 ([Kam2 $)$. $\left(\mathcal{B Z}^{w_{0}}, \mathrm{wt}, \varepsilon_{i}, \varphi_{i}, \widetilde{e}_{i}, \widetilde{f}_{i}\right)$ is a crystal in the sense of Definition 2.2.1, which is isomorphic to $B(\infty)$.

5.4. *-crystal structure on $e$-BZ data. By using Theorem 5.3.2, we can define a crystal structure on the set of $e$-BZ data $\mathcal{B Z}^{e}$ as follows. Recall the bijection $*: \mathcal{B Z}^{w_{0}} \stackrel{\sim}{\rightarrow} \mathcal{B Z}^{e}$ and its inverse which is also denoted by $*$. For $\mathbf{M}^{e} \in \mathcal{B Z}^{e}$, we set

$$
\operatorname{wt}\left(\mathbf{M}^{e}\right)=\operatorname{wt}\left(\mathbf{M}^{e *}\right), \quad \varepsilon_{i}^{*}\left(\mathbf{M}^{e}\right)=\varepsilon_{i}\left(\mathbf{M}^{e *}\right), \quad \varphi_{i}^{*}\left(\mathbf{M}^{e}\right)=\varphi_{i}\left(\mathbf{M}^{e *}\right) .
$$

Here we remark that $\mathbf{M}^{e *}$ is a $w_{0}$-BZ datum and the right hand sides are already defined. The Kashiwara operators $\widetilde{e}_{i}^{*}, \tilde{f}_{i}^{*}(i \in I)$ on $\mathcal{B Z}^{e}$ are defined by

$$
\widetilde{e}_{i}^{*}=* \circ \widetilde{e}_{i} \circ *, \quad \widetilde{f}_{i}^{*}=* \circ \widetilde{f}_{i} \circ * .
$$

The following corollary is an easy consequence of Proposition 5.3 .1 and Theorem 5.3.2.

Corollary 5.4.1. (1) Let $\mathbf{M}^{e}=\left(M_{K}^{e}\right) \in \mathcal{B Z} \mathcal{Z}^{e}$ be an e-BZ datum. If $\varepsilon_{i}^{*}\left(\mathbf{M}^{e}\right)>0$, there exists a unique e-BZ datum which is denoted by $\widetilde{e}_{i}^{*} \mathbf{M}^{e}$ such that

(i) $\left(\widetilde{e}_{i}^{*} \mathbf{M}^{e}\right)_{[1, i]^{c}}=M_{[1, i]^{c}}^{e}+1$,

(ii) $\left(\widetilde{e}_{i}^{*} \mathbf{M}^{e}\right)_{K}=M_{K}^{e}$ for all $K \in \mathcal{M}_{n}^{\times} \backslash \mathcal{M}_{n}^{\times}(i)^{*}$.

Here $\mathcal{M}_{n}^{\times}(i)^{*}=\left\{K \in \mathcal{M}_{n}^{\times} \mid i \notin K\right.$ and $\left.i+1 \in K\right\} \subset \mathcal{M}_{n}^{\times}$.

(2) There exists a unique a unique e-BZ datum which is denoted by $\widetilde{f}_{i}^{*} \mathbf{M}^{e}$ such that

(iii) $\left(\tilde{f}_{i}^{*} \mathbf{M}^{e}\right)_{[1, i]^{c}}=M_{[1, i]^{c}}^{e}-1$,

(iv) $\left(\tilde{f}_{i}^{*} \mathbf{M}^{e}\right)_{K}=M_{K}^{e}$ for all $K \in \mathcal{M}_{n}^{\times} \backslash \mathcal{M}_{n}^{\times}(i)^{*}$.

(3) $\left(\mathcal{B Z}^{e}, \mathrm{wt}, \varepsilon_{i}^{*}, \varphi_{i}^{*}, \widetilde{e}_{i}^{*}, \widetilde{f}_{i}^{*}\right)$ is a crystal in the sense of Definition 2.2.1, which is isomorphic to $B(\infty)$.

5.5. Anderson-Mirković conjecture. Let $\mathbf{M}^{w_{0}}=\left(M_{K}^{w_{0}}\right) \in \mathcal{B Z}^{w_{0}}$ be be a $w_{0}$-BZ datum. In Kam2, Kamnitzer gives the the explicit form of $\widetilde{f}_{i} \mathbf{M}^{w_{0}}$. We shall recall his result under the identification $\Gamma_{n} \cong \mathcal{M}_{n}^{\times}$.

Theorem 5.5.1 ([Kam2] $)$. For each $i \in I$, we have

$$
\left(\widetilde{f}_{i} \mathbf{M}^{w_{0}}\right)_{K}= \begin{cases}\min \left\{M_{K}^{w_{0}}, M_{s_{i} K}^{w_{0}}+c_{i}\left(\mathbf{M}^{w_{0}}\right)\right\} & \left(K \in \mathcal{M}_{n}^{\times}(i)\right), \\ M_{K}^{w_{0}} & \text { (otherwise) } .\end{cases}
$$

Here $c_{i}\left(\mathbf{M}^{w_{0}}\right)=M_{[1, i]}^{w_{0}}-M_{[1, i+1] \backslash\{i\}}^{w_{0}}-1$.

Remark . (1) If $K=[1, i]$, then we have $\left(\widetilde{f}_{i} \mathbf{M}^{w_{0}}\right)_{[1, i]}=M_{[1, i]}^{w_{0}}-1$. Indeed, $[1, i]$ is an element of $\mathcal{M}_{n}^{\times}(i)$. Since $s_{i}[1, i]=[1, i+1] \backslash\{i\}$, we have

$$
\begin{aligned}
\left(\widetilde{f}_{i} \mathbf{M}^{w_{0}}\right)_{[1, i]} & =\min \left\{M_{[1, i]}^{w_{0}}, M_{[1, i+1] \backslash\{i\}}^{w_{0}}+M_{[1, i]}^{w_{0}}-M_{[1, i+1] \backslash\{i\}}^{w_{0}}-1\right\} \\
& =\min \left\{M_{[1, i]}^{w_{0}}, M_{[1, i]}^{w_{0}}-1\right\} \\
& =M_{[1, i]}^{w_{0}}-1 .
\end{aligned}
$$


(2) As we already mentioned in the introduction, the above formula is conjectured by Anderson and Mirković (unpublished) (See [Kam2]). So it is called the Anderson-Mirković (AM for short) conjecture.

By using the above formula, we can also calculate the explicit form of the $\widetilde{f}_{i}^{*}$-action on an $e$-BZ datum.

Corollary 5.5.2. For $\mathbf{M}^{e}=\left(M_{K}^{e}\right) \in \mathcal{B Z}^{e}$ we have

$$
\left(\widetilde{f_{i}^{*}} \mathbf{M}^{e}\right)_{K}= \begin{cases}\min \left\{M_{K}^{e}, M_{s_{i} K}^{e}+c_{i}^{*}\left(\mathbf{M}^{e}\right)\right\} & \left(K \in \mathcal{M}_{n}^{\times}(i)^{*}\right) \\ M_{K}^{e} & \text { (otherwise) }\end{cases}
$$

Here $c_{i}^{*}\left(\mathbf{M}^{e}\right)=M_{[1, i]^{c}}^{e}-M_{([1, i+1] \backslash\{i\})^{c}}^{e}-1=M_{[i+1, n+1]}^{e}-M_{\{i\} \cup[i+2, n+1]}^{e}-1$.

5.6. Comparison. As we explained above both $\mathcal{B}$ (with the $*$-crystal structure) and $\mathcal{B Z}^{e}$ are crystal which are isomorphic to $B(\infty)$. Therefore, as abstract crystals, they are isomorphic. In this subsection we will construct an explicit isomorphism form $\mathcal{B}$ to $\mathcal{B Z}^{e}$.

Following [BFZ, we introduce a notion of $K$-tableau for a Maya diagram $K \in \mathcal{M}_{n}^{\times}$.

Definition 5.6.1. Let $K=\left\{k_{1}<k_{2}<\cdots<k_{l}\right\} \in \mathcal{M}_{n}^{\times}$be a Maya diagram. For such $K$, we define a $K$-tableau as an upper-triangular matrix $C=\left(c_{p, q}\right)_{1 \leq p \leq q \leq l}$ with integer entries satisfying

$$
c_{p, p}=k_{p} \quad(1 \leq p \leq l)
$$

and the usual monotonicity conditions for semi-standard tableaux:

$$
c_{p, q} \leq c_{p, q+1}, \quad c_{p, q}<c_{p+1, q}
$$

For a giving $\mathbf{i}_{0}$-Lusztig datum $\mathbf{a}=\left(a_{i, j}\right) \in \mathcal{B}$, let $\mathbf{M}(\mathbf{a})=\left(M_{K}(\mathbf{a})\right)_{K \in \mathcal{M}_{n}^{\times}}$be a collection of integers defined by

$$
M_{K}(\mathbf{a})=-\sum_{j=1}^{l} \sum_{i=1}^{k_{j}-1} a_{i, k_{j}}+\min \left\{\sum_{1 \leq p<q \leq l} a_{c_{p, q}, c_{p, q}+(q-p)} \mid C=\left(c_{p, q}\right) \text { is a } K \text {-tableau }\right\}
$$

and denote the map $\mathbf{a} \mapsto \mathbf{M}(\mathbf{a})$ by $\Psi$.

Proposition 5.6.2 ([BFZ $])$. For any $\mathbf{a} \in \mathcal{B}, \Psi(\mathbf{a})=\mathbf{M}(\mathbf{a})$ is an e-BZ datum. Moreover $\Psi: \mathcal{B} \rightarrow \mathcal{B Z}^{e}$ is a bijection.

In this article, we prove the next theorem.

Theorem 5.6.3. The bijection $\Psi: \mathcal{B} \rightarrow \mathcal{B Z}^{e}$ is an isomorphism of crystals with respect to $*$-crystal structures.

To prove this theorem, it is enough to show the next two lemmas.

Lemma 5.6.4. For any $\mathbf{a} \in \mathcal{B}$, we have

$$
\operatorname{wt}(\mathbf{M}(\mathbf{a}))=\operatorname{wt}(\mathbf{a}), \quad \varepsilon_{i}^{*}(\mathbf{M}(\mathbf{a}))=\varepsilon_{i}^{*}(\mathbf{a}), \quad \varphi_{i}^{*}(\mathbf{M}(\mathbf{a}))=\varphi_{i}^{*}(\mathbf{a}) .
$$

Lemma 5.6.5. For any $\mathbf{a} \in \mathcal{B}$, we have

$$
\widetilde{e}_{i}^{*}(\mathbf{M}(\mathbf{a}))=\mathbf{M}\left(\widetilde{e}_{i}^{*} \mathbf{a}\right), \quad \widetilde{f}_{i}^{*}(\mathbf{M}(\mathbf{a}))=\mathbf{M}\left(\widetilde{f}_{i}^{*} \mathbf{a}\right) .
$$

Here we set $\mathbf{M}(0)=0$. 
Proof of Lemma 5.6.4. Firstly let us compute $\operatorname{wt}(\mathbf{M}(\mathbf{a}))$. Since $\mathbf{M}(\mathbf{a})$ is an $e$-BZ datum we have

$$
\begin{aligned}
\operatorname{wt}(\mathbf{M}(\mathbf{a})) & =\sum_{i \in I} M_{[1, i]^{c}}(\mathbf{a}) \alpha_{i} \\
& =\sum_{i \in I} M_{[i+1, n+1]}(\mathbf{a}) \alpha_{i} .
\end{aligned}
$$

For $K=[i+1, n+1]$, there exist a unique $K$-tableau

$$
C=\left(c_{p, q}\right)_{1 \leq p \leq q \leq n+1-i}=\left(\begin{array}{ccccc}
i+1 & i+1 & \cdots & \cdots & i+1 \\
& i+2 & i+2 & \cdots & i+2 \\
& & \ddots & \ddots & \vdots \\
& & & n & n \\
& & & & n+1
\end{array}\right) .
$$

That is, $c_{p, q}=i+p(1 \leq p \leq q \leq n+1-i)$. Therefore, for any $i \in I$, we have

$$
\begin{aligned}
M_{[i+1, n+1]} & =-\sum_{t=i+1}^{n+1} \sum_{s=1}^{t-1} a_{s, t}+\sum_{1 \leq p<q \leq n+1-i} a_{i+p, i+p+(q-p)} \\
& =-\sum_{t=i+1}^{n+1} \sum_{s=1}^{i} a_{s, t} \\
& =-m_{i} .
\end{aligned}
$$

Here we set $m_{0}=m_{n+1}=0$. This equalities says that $\operatorname{wt}(\mathbf{M}(\mathbf{a}))=\operatorname{wt}(\mathbf{a})$.

Nextly let us calculate $\varepsilon_{i}^{*}(\mathbf{M}(\mathbf{a}))$. We have

$$
\begin{aligned}
\varepsilon_{i}^{*}(\mathbf{M}(\mathbf{a})) & =\varepsilon_{i}\left(\mathbf{M}(\mathbf{a})^{*}\right) \\
& =-M_{[i+1, n+1]}(\mathbf{a})-M_{\{i\} \cup[i+2, n+1]}(\mathbf{a})+M_{[i+2, n+1]}(\mathbf{a})+M_{[i, n+1]}(\mathbf{a}) .
\end{aligned}
$$

From the proof of the first formula we already know

$$
M_{[k+1, n+1]}(\mathbf{a})=-\sum_{t=k+1}^{n+1} \sum_{s=1}^{k} a_{s, t} \quad(k=i-1, i, i+1) .
$$

For $K=\{i\} \cup[i+2, n+1]$, the set of all $K$-tableaux is given by $\left\{C^{(r)}\right\}_{1 \leq r \leq n+1-i}$ where

$$
C^{(r)}=\left(c_{p, q}^{(r)}\right)_{1 \leq p \leq q \leq n+1-i}=\left(\begin{array}{ccccc}
i & c_{1,2}^{(r)} & \cdots & \cdots & c_{1, n+1-i}^{(r)} \\
& i+2 & i+2 & \cdots & i+2 \\
& & \ddots & \ddots & \vdots \\
& & & n & n \\
& & & & n+1
\end{array}\right)
$$

and

$$
c_{1, q}^{(r)}= \begin{cases}i & (2 \leq q \leq r), \\ i+1 & (r<q \leq n+1-i) .\end{cases}
$$

Since

$$
\sum_{q=2}^{n+1-i} a_{c_{1, q}^{(r)}, c_{1, q}^{(r)}+(q-1)}=\sum_{q=i+1}^{i+r-1} a_{i, q}+\sum_{q=i+r+1}^{n+1} a_{i+1, q},
$$


we have

$$
\begin{aligned}
M_{\{i\} \cup[i+2, n+1]}(\mathbf{a})= & -\sum_{s=1}^{i-1} a_{s, i}-\sum_{t=i+2}^{n+1} \sum_{s=1}^{t-1} a_{s, t}+\min _{1 \leq r \leq n+1-i}\left\{\sum_{1 \leq p<q \leq l} a_{c_{p, q}^{(r)}, c_{p, q}^{(r)}+(q-p)}\right\} \\
= & -\sum_{s=1}^{i-1} a_{s, i}-\sum_{t=i+2}^{n+1} \sum_{s=1}^{t-1} a_{s, t}+\sum_{q=i+3}^{n+1} \sum_{p=i+2}^{q-1} a_{p, q} \\
& +\min _{1 \leq r \leq n+1-i}\left\{\sum_{q=2}^{n+1-i} a_{c_{1, q}^{(r)}, c_{1, q}^{(r)}+(q-1)}\right\} \\
= & -\sum_{s=1}^{i-1} a_{s, i}-\sum_{t=i+2}^{n+1} \sum_{s=1}^{i+1} a_{s, t}+\min _{1 \leq r \leq n+1-i}\left\{\sum_{q=i+1}^{i+r-1} a_{i, q}+\sum_{q=i+r+1}^{n+1} a_{i+1, q}\right\} .
\end{aligned}
$$

Putting together all formulas, we have

$$
\begin{aligned}
\varepsilon_{i}^{*}(\mathbf{M}(\mathbf{a}))= & \sum_{t=i+1}^{n+1} \sum_{s=1}^{i} a_{s, t}-\sum_{t=i}^{n+1} \sum_{s=1}^{i-1} a_{s, t}-\sum_{t=i+2}^{n+1} \sum_{s=1}^{i+1} a_{s, t}+\sum_{s=1}^{i-1} a_{s, i}+\sum_{t=i+2}^{n+1} \sum_{s=1}^{i+1} a_{s, t} \\
& -\min _{1 \leq r \leq n+1-i}\left\{\sum_{q=i+1}^{i+r-1} a_{i, q}+\sum_{q=i+r+1}^{n+1} a_{i+1, q}\right\} \\
& =\sum_{t=i+1}^{n+1} a_{i, t}-\min _{1 \leq r \leq n+1-i}\left\{\sum_{q=i+1}^{i+r-1} a_{i, q}+\sum_{q=i+r+1}^{n+1} a_{i+1, q}\right\} \\
& =\max _{1 \leq r \leq n+1-i}\left\{\sum_{q=i+r}^{n+1} a_{i, q}-\sum_{q=i+r+1}^{n+1} a_{i+1, q}\right\} \\
& =\max _{1 \leq r \leq n+1-i}\left\{A_{i-1+r}^{*(i)}(\mathbf{a})\right\} \\
& =\varepsilon_{i}^{*}(\mathbf{a}) .
\end{aligned}
$$

Finally let us prove $\varphi_{i}^{*}(\mathbf{M}(\mathbf{a}))=\varphi_{i}^{*}(\mathbf{a})$. But it is clear by the first and second formulas.

We can prove Lemma 5.6.5 by direct calculation. But in this article we give another proof by using a Lagrangian constriction of $B(\infty)$, which we will explain later. (See Subsection 7.3.)

\section{Quivers OF TYPE $A_{n}$}

6.1. Quivers and their representations. Let $(I, H)$ be the double quiver of type $A_{n}$. Here $I=\{1,2, \cdots, n\}$ is the set of vertices and $H$ is the set of arrows. If $\tau \in H$ is the arrow from $i$ to $j$, we denote $\operatorname{out}(\tau)=i$ and $\operatorname{in}(\tau)=j$. For that $\tau \in H$, let $\bar{\tau}$ be the arrow from $j$ to $i$. The map $\tau \mapsto \bar{\tau}$ defines an involution of $H$. An orientation $\Omega$ is a subset of $H$ such that $\Omega \cap \bar{\Omega}=\phi$ and $\Omega \cup \bar{\Omega}=H$. Then $(I, \Omega)$ is a Dynkin quiver of type $A_{n}$.

Let $\mathbf{V}=(V, B)$ be a representation of the quiver $(I, \Omega)$. Here $V=\oplus_{i \in I} V_{i}$ be a finite dimensional $I$-graded complex vector space with the dimension vector $\operatorname{dim} V=$ $\left(\operatorname{dim}_{\mathbb{C}} V_{i}\right)_{i \in I} \in \mathbb{Z}_{\geq 0}^{I}$, and $B=\left(B_{\tau}\right)_{\tau \in \Omega}$ is a collection of linear maps $B_{\tau}: V_{\text {out }(\tau)} \rightarrow V_{\operatorname{in}(\tau)}$. We denote by $M \Omega$ the category of representations of the quiver $(I, \Omega)$. Let $\mathbf{V}=(V, B)$, $\mathbf{V}^{\prime}=\left(V^{\prime}, B^{\prime}\right) \in M \Omega$. A morphism $\phi=\left(\phi_{i}\right)_{i \in I}$ form $\mathbf{V}$ to $\mathbf{V}^{\prime}$ is a collection of linear 
maps $\phi_{i}: V_{i} \rightarrow V_{i}^{\prime}$ such that $\phi_{\operatorname{in}(\tau)} B_{\tau}=B_{\tau}^{\prime} \phi_{\text {out }(\tau)}$ for any $\tau \in \Omega$. It is well-known that $M \Omega$ is a Krull-Schmidt category. That is, any object in $M \Omega$ has a unique indecomposable decomposition. For $i \in I$ let $\mathbf{e}(i ; \Omega)$ be a representation of $(I, \Omega)$ defined by $V_{i}=\mathbb{C}$ and $V_{j}=0$ for $j \neq i$. This is simple and any simple representation isomorphic to $\mathbf{e}(i ; \Omega)$, for a unique $i$.

Assume that $i \in I$ is a sink (resp. a source) of an orientation $\Omega$. That is, there is no arrow $\tau \in \Omega$ such that $\operatorname{out}(\tau)=i$ (resp. $\operatorname{in}(\tau)=i$ ). We denote by $\operatorname{sink}(\Omega)$ (resp. source $(\Omega)$ ) the set of all sink (resp. source) vertices. Let $s_{i} \Omega$ be the orientation obtained from $\Omega$ by reversing each arrow $\tau$ such that $\operatorname{in}(\tau)=i(\operatorname{resp} . \operatorname{out}(\tau)=i)$.

Definition 6.1.1. Fix an orientation $\Omega$. A reduced word $\mathbf{i}=\left(i_{1}, \cdots, i_{N}\right)$ of $w_{0}$ is said to be adapted to $\Omega$ if $i_{k}$ is a sink of $\Omega_{k}=s_{i_{k-1}} \cdots s_{1} \Omega$ for $1 \leq k \leq N$.

For a representation of a quiver $\mathbf{V}=(V, B)$, we set $\operatorname{dim} \mathbf{V}=\operatorname{dim} V$. From now on, we identify the dimension vector $\operatorname{dim} V=\left(\operatorname{dim}_{\mathbb{C}} V_{i}\right)_{i \in I} \in \mathbb{Z}_{\geq 0}^{I}$ with an element of $Q_{+}=$ $\oplus_{i \in I} \mathbb{Z}_{\geq 0} \alpha_{i}$ by

$$
\left(\operatorname{dim}_{\mathbb{C}} V_{i}\right)_{i \in I} \mapsto \sum_{i \in I}\left(\operatorname{dim}_{\mathbb{C}} V_{i}\right) \alpha_{i} .
$$

Proposition 6.1.2. [L1] (1) For a giving orientation $\Omega$, there exist a reduced word $\mathbf{i}$ of $w_{0}$ adapted to $\Omega$.

(2) For each $\beta \in \Delta^{+}$, there is a unique indecomposable representation (up to isomorphism $) \mathbf{e}(\beta ; \Omega)$ such that $\operatorname{dim} \mathbf{e}(\beta ; \Omega)=\beta$. Moreover any indecomposable representation is isomorphic to $\mathbf{e}(\beta ; \Omega)$ for a unique $\beta$ (Gabriel's theorem).

(3) If $\beta>_{\mathbf{i}} \beta^{\prime}$, we have $\operatorname{Hom}_{M \Omega}\left(\mathbf{e}(\beta ; \Omega), \mathbf{e}\left(\beta^{\prime} ; \Omega\right)\right)=0$.

6.2. Orientations arising from Maya diagrams. Any Maya diagram $K \in \mathcal{M}_{n}^{\times}$can be written as a disjoint union of intervals

$$
\begin{gathered}
K=\left[s_{1}+1, t_{1}\right] \sqcup\left[s_{2}+1, t_{2}\right] \sqcup \cdots \sqcup\left[s_{l}+1, t_{l}\right] \\
\left(0 \leq s_{1}<t_{1}<s_{2}<t_{2}<\cdots<s_{l}<t_{l} \leq n+1\right) ;
\end{gathered}
$$

the interval $K_{m}=\left[s_{m}+1, t_{m}\right](1 \leq m \leq l)$ will be called the $m$-th component of $K$. Define two subsets out $(K)$ and $\operatorname{in}(K)$ of $[1, n]$ by

$$
\operatorname{out}(K)=\left\{t_{m} \mid 1 \leq m \leq l\right\} \cap[1, n], \quad \operatorname{in}(K)=\left\{s_{m} \mid 1 \leq m \leq l\right\} \cap[1, n] .
$$

We remark that $\operatorname{out}(K) \cap \operatorname{in}(K)=\phi$. Introduce two subsets $I_{t}$ and $I_{s}$ as follows:

$$
\begin{gathered}
I_{t}= \begin{cases}\operatorname{out}(K) \cup\{1, n\} & \left(s_{1} \geq 2, t_{l}=n+1\right), \\
\operatorname{out}(K) \cup\{1\} & \left(s_{1} \geq 2, t_{l} \leq n\right), \\
\operatorname{out}(K) \cup\{n\} & \left(s_{1} \leq 1, t_{l}=n+1\right), \\
\operatorname{out}(K) & \left(s_{1} \leq 1, t \leq n\right) .\end{cases} \\
I_{s}= \begin{cases}\operatorname{in}(K) \cup\{1, n\} & \left(s_{1}=0, t_{l} \leq n-1\right), \\
\operatorname{in}(K) \cup\{1\} & \left(s_{1}=0, t_{l} \geq n\right), \\
\operatorname{in}(K) \cup\{n\} & \left(s_{1} \geq 1, t_{l} \leq n-1\right), \\
\operatorname{in}(K) & \left(s_{1} \geq 1, t_{l} \geq n\right)\end{cases}
\end{gathered}
$$

Definition 6.2.1. (1) In the above setting, there exist a unique orientation $\Omega(K)$ so that source $(\Omega(K))=I_{t}$ and $\operatorname{sink}(\Omega(K))=I_{s}$. We call $\Omega(K)$ the orientation arising from $a$ Maya diagram $K \in \mathcal{M}_{n}^{\times}$.

(2) Let $s_{K}=\min \{k \mid k \notin K\}$ and $t_{K}=\max \{k \mid k \in K\}$. Define $\beta_{K} \in \Delta^{+} \cup\{0\}$ by

$$
\beta_{K}= \begin{cases}\alpha_{s_{K}}+\alpha_{s_{K}+1}+\cdots+\alpha_{t_{K}-1} & \left(s_{K}<t_{K}\right), \\ 0 & \text { (otherwise) }\end{cases}
$$


and we call it the characterizing positive root of a Maya diagram $\mathrm{K}$.

Remark . (1) In general, we have

$$
\operatorname{out}(K) \subset \operatorname{source}(\Omega(K)), \quad \operatorname{in}(K) \subset \operatorname{sink}(\Omega(K)) .
$$

(2) The characterizing positive root $\beta_{K}=0$ if and only if $K=\left[1, t_{1}\right]$ for some $1 \leq t_{1} \leq n$.

Example 6.2.2. Let $n=17$ and $K=[3,4] \sqcup[7,8] \sqcup[10,12] \sqcup[14,15]$. Then we have

$$
\operatorname{out}(K)=\{4,8,12,15\}, \quad \operatorname{in}(K)=\{2,6,9,13\} .
$$

Since $s_{1}=2$ and $t_{l}=t_{4}=15$, we have

$$
I_{t}=\operatorname{out}(K) \cup\{1\}=\{1,4,8,12,15\}, \quad I_{s}=\operatorname{in}(K) \cup\{17\}=\{2,6,9,13,17\} .
$$

In this case, the orientation $\Omega(K)$ is given as follows:

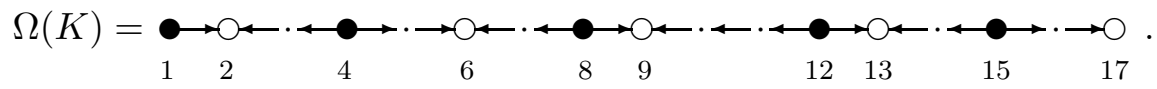

Here $\circ$ is a sink and $\bullet$ is a source

Since $s_{K}=1$ and $t_{K}=15$, the characterizing positive root $\beta_{K}$ is given by

$$
\beta_{K}=\sum_{i=1}^{14} \alpha_{i}
$$

6.3. From Lusztig data to $e-\mathbf{B Z}$ data. Let $\mathbf{i}$ be a reduced word adapted to the orientation $\Omega(K)$ and consider the set of all $\mathbf{i}$-Lusztig data

$$
\mathcal{B}^{\mathbf{i}}=\left\{\mathbf{a}^{\mathbf{i}}=\left(a_{i, j}^{\mathbf{i}}\right)_{(i, j) \in \Pi} \mid a_{i, j}^{\mathbf{i}} \in \mathbb{Z}_{\geq 0}\right\} .
$$

Recall the identification $\Delta^{+} \stackrel{\sim}{\rightarrow} \Pi$ (see Subsection 2.4) and denote the image of $\beta \in \Delta^{+}$ by $\left(i_{\beta}, j_{\beta}\right) \in \Pi$. Set $\mathbf{e}\left(\left(i_{\beta}, j_{\beta}\right) ; \Omega(K)\right)=\mathbf{e}(\beta ; \Omega(K))$. Then, for each $\mathbf{V} \in M \Omega(K)$, there is a unique $\mathbf{a}^{\mathbf{i}} \in \mathcal{B}^{\mathbf{i}}$ such that $\mathbf{V}$ is isomorphic to $\mathbf{V}\left(\mathbf{a}^{\mathbf{i}}\right)$. Here

$$
\mathbf{V}\left(\mathbf{a}^{\mathbf{i}}\right)=\underset{(i, j) \in \Pi}{\oplus} \mathbf{e}((i, j) ; \Omega(K))^{\oplus a_{i, j}^{\mathbf{i}}} .
$$

We introduce a non-positive integer

$$
M_{K}\left(\mathbf{V}\left(\mathbf{a}^{\mathbf{i}}\right)\right)=-\operatorname{dim}_{\mathbb{C}} \operatorname{Hom}_{M \Omega(K)}\left(\mathbf{V}\left(\mathbf{a}^{\mathbf{i}}\right), \mathbf{e}\left(\beta_{K} ; \Omega(K)\right)\right) .
$$

Lemma 6.3.1. (1) We have

$$
M_{K}\left(\mathbf{V}\left(\mathbf{a}^{\mathbf{i}}\right)\right)=-\sum_{(i, j) \in \Pi ; i \notin K, j \in K} a_{i, j}^{\mathbf{i}} .
$$

(2) Denote $\mathbf{V}\left(\mathbf{a}^{\mathbf{i}}\right)=\left(\oplus_{i} V_{i},\left(B_{\tau}\right)_{\tau \in \Omega(K)}\right)$. Then we have

$$
\sum_{(i, j) \in \Pi ; i \notin K, j \in K} a_{i, j}^{\mathbf{i}}=\operatorname{dim}_{\mathbb{C}} \operatorname{Coker}\left(\underset{k \in \operatorname{out}(K)}{\oplus} V_{k} \stackrel{\oplus B_{q}}{\longrightarrow} \underset{l \in \operatorname{in}(K)}{\oplus} V_{l}\right) .
$$

Here $\sigma$ is a path in $\Omega(K)$ form some $k \in \operatorname{out}(K)$ to some $l \in \operatorname{in}(K)$

The proof of this lemma will be given in the next subsection.

The next proposition is a easy consequence of the results of Berenstein, Fomin and Zelevinsky [BFZ]. 
Proposition 6.3.2. Let $\mathbf{i}$ be a reduced word adapted to the orientation $\Omega(K)$ and $\mathbf{a} \in \mathcal{B}$ an $\mathbf{i}_{0}$-Lusztig datum. Set $\mathbf{a}^{\mathbf{i}}=R_{\mathbf{i}_{0}}^{\mathbf{i}}(\mathbf{a})$. Here $R_{\mathbf{i}_{0}}^{\mathbf{i}}$ is the transition map from $\mathbf{i}_{0}$ to $\mathbf{i}$. Then we have

$$
M_{K}(\mathbf{a})=-\sum_{(i, j) \in \Pi ; i \notin K, j \in K} a_{i, j}^{\mathbf{i}} .
$$

Here $\mathbf{M}(\mathbf{a})=\left(M_{K}(\mathbf{a})\right)_{K \in \mathcal{M}_{n}^{\times}}$is the e-BZ datum defined in 5.6.

Combining the above results, we have the following corollary:

Corollary 6.3.3. In the above setting, we have

$$
M_{K}(\mathbf{a})=M_{K}\left(\mathbf{V}\left(\mathbf{a}^{\mathbf{i}}\right)\right)=-\operatorname{dim}_{\mathbb{C}} \operatorname{Coker}\left(\underset{k \in \operatorname{out}(K)}{\oplus} V_{k} \stackrel{\oplus B_{q}}{\longrightarrow} \underset{l \in \operatorname{in}(K)}{\oplus} V_{l}\right) .
$$

6.4. Proof of Lemma 6.3.1. Let us prove the formula (1). It is enough to show that

$$
\operatorname{dim}_{\mathbb{C}} \operatorname{Hom}_{M \Omega(K)}\left(\mathbf{e}((i, j) ; \Omega(K)), \mathbf{e}\left(\beta_{K} ; \Omega(K)\right)\right)= \begin{cases}1 & (i \notin K \text { and } j \in K), \\ 0 & \text { (otherwise). }\end{cases}
$$

Let us denote $\mathbf{e}((i, j) ; \Omega(K))=\left(\oplus V_{k}^{\prime},\left(B_{\tau}^{\prime}\right)\right)$ and $\mathbf{e}\left(\beta_{K} ; \Omega(K)\right)=\left(\oplus V_{k}^{\prime \prime},\left(B_{\tau}^{\prime \prime}\right)\right)$. Then

$$
V_{k}^{\prime}=\left\{\begin{array}{ll}
\mathbb{C} & (i \leq k \leq j-1), \\
0 & \text { (otherwise) }
\end{array} \quad \text { and } \quad V_{k}^{\prime \prime}= \begin{cases}\mathbb{C} & \left(s_{K} \leq k \leq t_{K}-1\right), \\
0 & \text { (otherwise) }\end{cases}\right.
$$

Firstly assume $i \notin K$ and $j \in K$. Since our quiver is of type $A_{n}$, the left hand side of (6.4.1) is less than 1. So it is enough to show that there is a non-trivial morphism form $\left(\oplus V_{k}^{\prime},\left(B_{\tau}^{\prime}\right)\right)$ to $\left(\oplus V_{k}^{\prime \prime},\left(B_{\tau}^{\prime \prime}\right)\right)$. By the assumption we have $s_{K} \leq i<j \leq t_{K}$. Therefore we can define a linear map $\psi=\left(\psi_{k}\right): \oplus V_{k}^{\prime} \rightarrow \oplus V_{k}^{\prime \prime}$ by

$$
\psi_{k}= \begin{cases}\operatorname{id}_{\mathbb{C}} & (i \leq k \leq j-1) \\ 0 & \text { (otherwise) }\end{cases}
$$

and it is easy to check that the above map is a non-trivial morphism of $M \Omega(K)$.

Secondly let us consider the case that $i \in K$ or $j \notin K$. The goal is to prove

$$
\operatorname{Hom}_{M \Omega(K)}\left(\left(\oplus V_{k}^{\prime},\left(B_{\tau}^{\prime}\right)\right),\left(\oplus V_{k}^{\prime \prime},\left(B_{\tau}^{\prime \prime}\right)\right)\right)=0 .
$$

Assume $i \in K$ and $s_{K}<i$. Then $i \geq 2, i+1 \leq t_{K}$ and there is an arrow $\tau_{1}$ form $i$ to $i-1$ in $\Omega(K)$. Let $\psi=\left(\psi_{k}\right) \in \operatorname{Hom}_{M \Omega(K)}\left(\left(\oplus V_{k}^{\prime},\left(B_{\tau}^{\prime}\right)\right),\left(\oplus V_{k}^{\prime \prime},\left(B_{\tau}^{\prime \prime}\right)\right)\right)$. Since $V_{i-1}^{\prime}=0$, we have $B_{\tau_{1}}^{\prime \prime} \psi_{i}=\psi_{i-1} B_{\tau_{1}}^{\prime}=0$. On the other hand, $B_{\tau_{1}}^{\prime \prime} \neq 0$ because $s_{K} \leq i-1$. Therefore we have $\psi_{i}=0$.

If $j=i+1$ or $t_{K}=i+1$, it means that the left hand side of (6.4.2) is equal to zero. So we may assume $j>i+1$ and $t_{K}>i+1$. However one can show that $\psi_{i+1}=0$. Indeed, if there is an arrow $\tau_{2}$ form $i$ to $i+1$ in $\Omega(K)$, then we have $\psi_{i+1} B_{\tau_{2}}^{\prime}=B_{\tau_{2}}^{\prime \prime} \psi_{i}$ with non-trivial $B_{\tau_{2}}^{\prime}$ and $B_{\tau_{2}}^{\prime \prime}$. Since $\psi_{i}=0$, we have $\psi_{i+1}=0$. On the other hand, if there is an arrow $\tau_{2}$ form $i+1$ to $i$ in $\Omega(K)$, then we have $\psi_{i} B_{\tau_{2}}^{\prime}=B_{\tau_{2}}^{\prime \prime} \psi_{i+1}$. Then we also have $\psi_{i+1}=0$. By repeating this method, we have $\psi_{k}=0$ for any $k \in I$.

For the other cases, we can show (6.4.2) by similar way.

We will give a proof of (2). Since $\mathbf{V}\left(\mathbf{a}^{\mathbf{i}}\right)=\oplus_{(i, j) \in \Pi} \mathbf{e}((i, j) ; \Omega(K))^{\oplus a_{i, j}^{\mathbf{i}} \text { is the indecom- }}$ posable decomposition, it is enough to prove that

$$
\operatorname{dim}_{\mathbb{C}} \operatorname{Coker}\left(\underset{k \in \operatorname{out}(K)}{\oplus} V_{k}^{\prime} \stackrel{\oplus B_{\sigma}^{\prime}}{\longrightarrow} \underset{l \in \operatorname{in}(K)}{\oplus} V_{l}^{\prime}\right)= \begin{cases}1 & (i \notin K \text { and } j \in K), \\ 0 & \text { (otherwise). }\end{cases}
$$

Recall the decomposition of $K$ :

$$
K=K_{1} \sqcup \cdots \sqcup K_{l}, \quad \text { where } K_{m}=\left[s_{m}+1, t_{m}\right] \quad(1 \leq m \leq l) .
$$


Firstly assume $i \notin K$ and $j \in K$. More precisely, we assume $t_{u-1}<i<s_{u}+1$ and $s_{v}+1 \leq j \leq t_{v}$ with $u \leq v$. Let $\sigma\left(t_{m-1} \rightarrow s_{m}\right)\left(\right.$ resp. $\left.\sigma\left(s_{m} \leftarrow t_{m}\right)\right)$ be the path form $t_{m-1}$ to $s_{m}$ (resp. from $t_{m}$ to $s_{m}$ ) in $\Omega(K)$. Then, by the definition, we have

$$
B_{\sigma\left(t_{m-1} \rightarrow s_{m}\right)}^{\prime}=\left\{\begin{array}{ll}
\operatorname{id}_{\mathbb{C}} & (u+1 \leq m \leq v), \\
0 & \text { (otherwise) },
\end{array} B_{\sigma\left(s_{m} \leftarrow t_{m}\right)}^{\prime}= \begin{cases}\operatorname{id}_{\mathbb{C}} & (u \leq m \leq v-1), \\
0 & \text { (otherwise) } .\end{cases}\right.
$$

Therefore we have

$$
\begin{aligned}
& \operatorname{dim}_{\mathbb{C}} \operatorname{Coker}\left(\underset{k \in \operatorname{out}(K)}{\oplus} V_{k}^{\prime} \stackrel{\oplus B_{a}^{\prime}}{\left.\underset{l \in \operatorname{in}(K)}{\oplus} V_{l}^{\prime}\right)}=\operatorname{dim}_{\mathbb{C}} \operatorname{Coker}\left(\underset{m=u}{\oplus-1} V_{t_{m}}^{\prime} \longrightarrow \underset{m=u}{\oplus} V_{s_{m}}^{\prime}\right)\right. \\
&=\operatorname{dim}_{\mathbb{C}} \operatorname{Coker}\left(\mathbb{C}^{v-u-1} \hookrightarrow \mathbb{C}^{v-u}\right) \\
&=1 .
\end{aligned}
$$

Secondly assume $i, j \in K$. Then there exist $u$ and $v$ with $u \leq v$ such that $i \in K_{u}$ and $j \in K_{v}$. In this case, we have

$$
B_{\sigma\left(t_{m-1} \rightarrow s_{m}\right)}^{\prime}=\left\{\begin{array}{ll}
\operatorname{id}_{\mathbb{C}} & (u+1 \leq m \leq v), \\
0 & \text { (otherwise) },
\end{array} B_{\sigma\left(s_{m} \leftarrow t_{m}\right)}^{\prime}= \begin{cases}\operatorname{id}_{\mathbb{C}} & (u+1 \leq m \leq v-1), \\
0 & \text { (otherwise) } .\end{cases}\right.
$$

Therefore we have

$$
\begin{aligned}
\operatorname{dim}_{\mathbb{C}} \operatorname{Coker}\left(\underset{k \in \operatorname{Out}(K)}{\oplus} V_{k}^{\prime} \stackrel{\oplus B_{g}^{\prime}}{\rightarrow} \underset{l \in \operatorname{in}(K)}{\oplus} V_{l}^{\prime}\right) & =\operatorname{dim}_{\mathbb{C}} \operatorname{Coker}\left(\underset{m=u}{\oplus-1} V_{t_{m}}^{\prime} \longrightarrow \underset{m=u+1}{\oplus} V_{s_{m}}^{\prime}\right) \\
& =\operatorname{dim}_{\mathbb{C}} \operatorname{Coker}\left(\mathbb{C}^{v-u-1} \stackrel{\sim}{\rightarrow} \mathbb{C}^{v-u-1}\right) \\
& =0 .
\end{aligned}
$$

For the other cases, we can prove that the left hand side of (6.4.3) equals to zero by similar arguments. Thus, the lemma is proved.

\section{LAGRANGIAN CONSTRUCTION OF CRYSTAL BASIS}

7.1. Varieties associated to quivers. For $\nu \in Q_{+}$, let $\mathcal{V}_{\nu}$ be the category of $I$-graded complex vector spaces $V$ with $\operatorname{dim} V=\nu$. For $V=\oplus_{i \in I} V_{i} \in \mathcal{V}_{\nu}$, introduce two complex vector spaces

$$
E_{V, \Omega}=\underset{\tau \in \Omega}{\oplus} \operatorname{Hom}_{\mathbb{C}}\left(V_{\text {out }(\tau)}, V_{\operatorname{in}(\tau)}\right), \quad X_{V}=\underset{\tau \in H}{\oplus} \operatorname{Hom}_{\mathbb{C}}\left(V_{\text {out }(\tau)}, V_{\operatorname{in}(\tau)}\right) .
$$

An element of $E_{V, \Omega}$ or $X_{V}$ will be denoted by $B=\left(B_{\tau}\right)$ where $B_{\tau} \in \operatorname{Hom}_{\mathbb{C}}\left(V_{\text {out }(\tau)}, V_{\operatorname{in}(\tau)}\right)$. Define a symplectic form $\omega$ on $X_{V}$ by

$$
\omega\left(B, B^{\prime}\right)=\sum_{\tau \in H} \varepsilon(\tau) \operatorname{tr}\left(B_{\bar{\tau}} B_{\tau}^{\prime}\right)
$$

where $\varepsilon(\tau)=1$ for $\tau \in \Omega$ and $\varepsilon(\tau)=-1$ for $\tau \in \bar{\Omega}$. We regard $X_{V}$ as the cotangent bundle $T^{*} E_{V, \Omega}$ of $E_{V, \Omega}$ via the symplectic form $\omega$.

The group $G_{V}=\prod_{i \in I} G L\left(V_{i}\right)$ acts on $E_{V, \Omega}$ and $X_{V}$ by

$$
G_{V} \ni g=\left(g_{i}\right):\left(B_{\tau}\right) \mapsto\left(g_{\text {in }(\tau)} B_{\tau} g_{\text {out }(\tau)}^{-1}\right) .
$$

Since the action of $G$ on $X_{V}$ preserves the symplectic form $\omega$, we can consider the corresponding moment map $\mu: X_{V} \rightarrow\left(\mathfrak{g}_{V}\right)^{*} \cong \mathfrak{g}_{V}$. Here $\mathfrak{g}_{V}=$ Lie $G_{V}$ and we identify $\mathfrak{g}_{V}$ with its dual via the Killing form. Set

$$
\Lambda_{V}=\mu^{-1}(0) .
$$


It is known that $\Lambda_{V}$ is a $G_{V^{-}}$-invariant closed Lagrangian subvariety of $X_{V}$. It is clear that, for $V, V^{\prime} \in \mathcal{V}_{\nu}$, there are natural isomorphisms $V \cong V^{\prime}, X_{V} \cong X_{V^{\prime}}$ and $\Lambda_{V} \cong \Lambda_{V^{\prime}}$. Hence we denote them $V(\nu), X(\nu)$ and $\Lambda(\nu)$, respectively.

Let $\operatorname{Irr} \Lambda(\nu)$ be a set of all irreducible components of $\Lambda(\nu)$. Since our quiver is of type $A_{n}$, there is a bijection from the set of all $G_{V(\nu)}$-orbits in $E_{V(\nu), \Omega}$ to $\operatorname{Irr} \Lambda(\nu)$ defined by $\mathcal{O} \mapsto \overline{T_{\mathcal{O}}^{*} E_{V(\nu), \Omega}}$. We remark that $E_{V(\nu), \Omega}$ has finitely many $G_{V(\nu)}$-orbits because our quiver is of type $A_{n}$.

For $B \in E_{V(\nu), \Omega}$, a pair $\mathbf{V}=(V(\nu), B)$ is nothing but a representation of a quiver $(I, \Omega)$ with a dimension vector $\nu$. Moreover there is a natural one to one correspondence between isomorphism classes of representations of a quiver $(I, \Omega)$ with a dimension vector $\nu$ and $G_{V(\nu)}$-orbits in $E_{V(\nu), \Omega}$.

Let $\Omega$ be an orientation and $\mathbf{i}$ a reduced word adapted to $\Omega$. As we mentioned before, for each $\mathbf{V}=(V(\nu), B) \in M \Omega$, there is a unique $\mathbf{i}$-Lusztig datum $\mathbf{a}^{\mathbf{i}} \in \mathcal{B}^{\mathbf{i}}$ such that $\mathbf{V}$ is isomorphic to

$$
\mathbf{V}\left(\mathbf{a}^{\mathbf{i}}\right)=\underset{(i, j) \in \Pi}{\oplus} \mathbf{e}((i, j) ; \Omega)^{\oplus a_{i, j}^{\mathbf{i}}}
$$

Let $\mathcal{O}_{\mathbf{a}^{\mathbf{i}}}$ be the $G_{V(\nu)}$-orbit of $E_{V(\nu), \Omega}$ through $\mathbf{V}\left(\mathbf{a}^{\mathbf{i}}\right)$. Denote $\Lambda_{\mathbf{a}^{\mathbf{i}}}=\overline{T_{\mathcal{O}_{\mathbf{a}^{\mathbf{i}}}^{*}} E_{V(\nu), \Omega}}$. Then we have a bijection $\Psi_{\mathbf{i}}: \mathcal{B}^{\mathbf{i}} \stackrel{\sim}{\rightarrow} \bigsqcup_{\nu \in Q^{-}} \operatorname{Irr} \Lambda(\nu)$ defined by $\mathbf{a}^{\mathbf{i}} \mapsto \Lambda_{\mathbf{a}^{\mathbf{i}}}$. Especially, consider the following special orientation

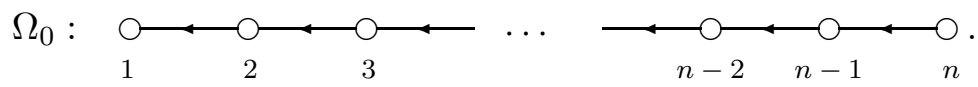

Then the lexicographically minimal reduced word $\mathbf{i}_{0}$ is adapted to $\Omega_{0}$. For $\mathbf{a} \in \mathcal{B}$, let $\mathcal{O}_{\mathbf{a}}$ be the corresponding orbit in $E_{V(\nu), \Omega_{0}}$ and $\Lambda_{\mathbf{a}}=\overline{T_{\mathcal{O}_{\mathbf{a}}}^{*} E_{V(\nu), \Omega_{0}}}$. We remark that

$$
\Lambda_{\mathbf{a}}=\Lambda_{\mathbf{a}^{\mathbf{i}}}
$$

where $\mathbf{i}$ is an arbitrarily reduced word and $\mathbf{a}^{\mathbf{i}}=R_{\mathbf{i}_{0}}^{\mathbf{i}}(\mathbf{a})$.

Remark . It seems to us that the formula (7.1.1) is known for experts. However the proof of it was not appeared until recently. A detailed proof was firstly given by Kimura in his Master thesis [Kim] (see Appendix A, in detail).

On the other hand, in 2010, Baumann and Kamnitzer give another explicit proof of it by using representation theory of preprojective algebra (see [BK]).

For $B \in X(\nu)$ we set

$$
M_{K}(B)=-\operatorname{dim}_{\mathbb{C}} \operatorname{Coker}\left(\underset{k \in \operatorname{out}(K)}{\oplus} V(\nu)_{k} \stackrel{\oplus B_{\sigma}}{\longrightarrow} \underset{l \in \operatorname{in}(K)}{\oplus} V(\nu)_{l}\right)
$$

and for $\Lambda \in \operatorname{Irr} \Lambda(\nu)$ define

$$
M_{K}(\Lambda)=M_{K}(B)
$$

by taking a generic point $B$ of $\Lambda$. By Corollary 6.3.3, we immediately have the following statement.

Corollary 7.1.1. Recall the setting of Corollary 6.3.3; let $\mathbf{i}$ be a reduced word which is adapted to the orientation $\Omega(K)$ and $\mathbf{a} \in \mathcal{B}$ an $\mathbf{i}_{0}$-Lusztig datum. Set $\mathbf{a}^{\mathbf{i}}=R_{\mathbf{i}_{0}}^{\mathbf{i}}(\mathbf{a})$. Then we have

$$
M_{K}(\mathbf{a})=M_{K}\left(\mathbf{V}\left(\mathbf{a}^{\mathbf{i}}\right)\right)=M_{K}\left(\Lambda_{\mathbf{a}^{\mathbf{i}}}\right) .
$$

Combining the above corollary with (7.1.1), we have

$$
M_{K}(\mathbf{a})=M_{K}\left(\Lambda_{\mathbf{a}}\right) \text {. }
$$


7.2. Lagrangian construction of $B(\infty)$. In this subsection we will give a review of Lagrangian construction of $B(\infty)$ following [KS].

Let $\nu, \nu^{\prime}, \bar{\nu} \in Q_{+}$with $\nu=\nu^{\prime}+\bar{\nu}$. Consider a diagram

$$
\Lambda\left(\nu^{\prime}\right) \times \Lambda(\bar{\nu}) \stackrel{q_{1}}{\longleftarrow} \Lambda\left(\nu^{\prime}, \bar{\nu}\right) \stackrel{q_{2}}{\longrightarrow} \Lambda(\nu) .
$$

Here $\Lambda\left(\nu^{\prime}, \bar{\nu}\right)$ is a variety of $\left(B, \phi^{\prime}, \bar{\phi}\right)$, where $B \in \Lambda(\nu)$ and $\phi^{\prime}=\left(\phi_{i}^{\prime}\right), \bar{\phi}=\left(\bar{\phi}_{i}\right)$ give an exact sequence

$$
0 \longrightarrow V\left(\nu^{\prime}\right)_{i} \stackrel{\phi_{i}^{\prime}}{\longrightarrow} V(\nu) \stackrel{\bar{\phi}_{i}}{\longrightarrow} V(\bar{\nu}) \longrightarrow 0
$$

such that $\operatorname{Im} \phi^{\prime}$ is stable by $B$. Hence $B$ induces $B^{\prime}: V\left(\nu^{\prime}\right) \rightarrow V\left(\nu^{\prime}\right)$ and $\bar{B}: V(\bar{\nu}) \rightarrow V(\bar{\nu})$. The maps $q_{1}$ and $q_{2}$ are defined by $q_{1}\left(B, \phi^{\prime}, \bar{\phi}\right)=\left(B^{\prime}, \bar{B}\right)$ and $q_{2}\left(B, \phi^{\prime}, \bar{\phi}\right)=B$, respectively.

For $i \in I$ and $\Lambda \in \operatorname{Irr} \Lambda(\nu)$, set

$$
\varepsilon_{i}(\Lambda)=\varepsilon_{i}(B) \text { and } \varepsilon_{i}^{*}(\Lambda)=\varepsilon_{i}^{*}(B),
$$

where $B$ is a general point of $\Lambda$ and

$$
\begin{aligned}
& \varepsilon_{i}(B)=\operatorname{dim}_{\mathbb{C}} \operatorname{Coker}\left(\underset{\tau ; \operatorname{in}(\tau)=i}{\oplus} V(\nu)_{\text {out }(\tau)} \stackrel{\oplus B_{\mathcal{T}}}{\longrightarrow} V(\nu)_{i}\right),
\end{aligned}
$$

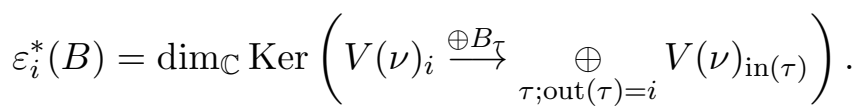

For $k, l \in \mathbb{Z}_{\geq 0}$, we define

$$
(\operatorname{Irr} \Lambda(\nu))_{i, k}=\left\{\Lambda \in \operatorname{Irr} \Lambda(\nu) \mid \varepsilon_{i}(\Lambda)=k\right\} \text { and }(\operatorname{Irr} \Lambda(\nu))_{i}^{l}=\left\{\Lambda \in \operatorname{Irr} \Lambda(\nu) \mid \varepsilon_{i}^{*}(\Lambda)=l\right\} .
$$

Assume $\bar{\nu}=c \alpha_{i}$ (resp. $\left.\nu^{\prime}=c \alpha_{i}\right)$ for $c \in \mathbb{Z}_{\geq 0}$. Since $\Lambda\left(c \alpha_{i}\right)=\{0\}$, we have the following diagrams as special cases of (7.2.1):

$$
\begin{gathered}
\Lambda\left(\nu^{\prime}\right) \cong \Lambda\left(\nu^{\prime}\right) \times \Lambda\left(c \alpha_{i}\right) \stackrel{q_{1}}{\longleftarrow} \Lambda\left(\nu^{\prime}, c \alpha_{i}\right) \stackrel{q_{2}}{\longrightarrow} \Lambda(\nu), \\
\Lambda(\bar{\nu}) \cong \Lambda\left(c \alpha_{i}\right) \times \Lambda(\bar{\nu}) \stackrel{q_{1}}{\longleftarrow} \Lambda\left(c \alpha_{i}, \bar{\nu}\right) \stackrel{q_{2}}{\longrightarrow} \Lambda(\nu) .
\end{gathered}
$$

It is known that the diagrams (7.2.2) and (7.2.3) induce bijections

$$
\widetilde{e}_{i}^{\max }:(\operatorname{Irr} \Lambda(\nu))_{i, c} \stackrel{\sim}{\rightarrow}\left(\operatorname{Irr} \Lambda\left(\nu^{\prime}\right)\right)_{i, 0} \quad \text { and } \quad \widetilde{e}_{i}^{* \max }:(\operatorname{Irr} \Lambda(\nu))_{i}^{c} \stackrel{\sim}{\rightarrow}(\operatorname{Irr} \Lambda(\bar{\nu}))_{i}^{0},
$$

respectively. We introduce maps

$$
\widetilde{e}_{i}, \widetilde{e}_{i}^{*}: \bigsqcup_{\nu \in Q_{+}} \operatorname{Irr} \Lambda(\nu) \rightarrow \bigsqcup_{\nu \in Q_{+}} \operatorname{Irr} \Lambda(\nu) \sqcup\{0\} \quad \text { and } \quad \tilde{f}_{i}, \widetilde{f}_{i}^{*}: \bigsqcup_{\nu \in Q_{+}} \operatorname{Irr} \Lambda(\nu) \rightarrow \bigsqcup_{\nu \in Q_{+}} \operatorname{Irr} \Lambda(\nu)
$$

as follows: if $c>0$ we define

$$
\begin{array}{ll}
\widetilde{e}_{i}:(\operatorname{Irr} \Lambda(\nu))_{i, c} & \stackrel{\sim}{\longrightarrow}\left(\operatorname{Irr} \Lambda\left(\nu^{\prime}\right)\right)_{i, 0} \stackrel{\sim}{\longrightarrow}\left(\operatorname{Irr} \Lambda\left(\nu+\alpha_{i}\right)\right)_{i, c-1}, \\
\widetilde{e}_{i}^{*}:(\operatorname{Irr} \Lambda(\nu))_{i}^{c} & \stackrel{\sim}{\longrightarrow}(\operatorname{Irr} \Lambda(\bar{\nu}))_{i}^{0} \stackrel{\sim}{\longrightarrow}\left(\operatorname{Irr} \Lambda\left(\nu+\alpha_{i}\right)\right)_{i}^{c-1}
\end{array}
$$

and $\widetilde{e}_{i} \Lambda=0$ and $\widetilde{e}_{i}^{*} \Lambda^{\prime}=0$ for $\Lambda \in(\operatorname{Irr} \Lambda(\nu))_{i, 0}$ and $\Lambda^{\prime} \in(\operatorname{Irr} \Lambda(\nu))_{i}^{0}$, respectively. Define

$$
\begin{aligned}
& \tilde{f}_{i}: \quad(\operatorname{Irr} \Lambda(\nu))_{i, c} \stackrel{\sim}{\longrightarrow}\left(\operatorname{Irr} \Lambda\left(\nu^{\prime}\right)\right)_{i, 0} \stackrel{\sim}{\longrightarrow}\left(\operatorname{Irr} \Lambda\left(\nu-\alpha_{i}\right)\right)_{i, c+1}, \\
& \tilde{f}_{i}^{*}:(\operatorname{Irr} \Lambda(\nu))_{i}^{c} \stackrel{\sim}{\longrightarrow}(\operatorname{Irr} \Lambda(\bar{\nu}))_{i}^{0} \stackrel{\sim}{\longrightarrow}\left(\operatorname{Irr} \Lambda\left(\nu-\alpha_{i}\right)\right)_{i}^{c+1} .
\end{aligned}
$$

Theorem 7.2.1. [KS] (1) For $\Lambda \in \operatorname{Irr} \Lambda(\nu)$, we set wt $\Lambda=-\nu, \varphi_{i}(\Lambda)=\varepsilon_{i}(\Lambda)+\left\langle h_{i}\right.$, wt $\left.\Lambda\right\rangle$. Then $\left(\bigsqcup_{\nu \in Q_{+}} \operatorname{Irr} \Lambda(\nu)\right.$; wt, $\left.\varepsilon_{i}, \varphi_{i}, \widetilde{e}_{i}, \widetilde{f}_{i}\right)$ is a crystal isomorphic to $\left(B(\infty)\right.$; wt, $\left.\varepsilon_{i}, \varphi_{i}, \widetilde{e}_{i}, \widetilde{f}_{i}\right)$. More precisely, the explicit form of the isomorphism $\Phi: B(\infty) \stackrel{\sim}{\rightarrow} \bigsqcup_{\nu \in Q_{+}} \operatorname{Irr} \Lambda(\nu)$ is given by $\Phi=\Psi_{\mathbf{i}} \circ \Xi_{\mathbf{i}}^{-1}$. 
(2) Set $\varphi_{i}^{*}(\Lambda)=\varepsilon_{i}^{*}(\Lambda)+\left\langle h_{i}, \mathrm{wt} \Lambda\right\rangle$. Then $\left(\bigsqcup_{\nu \in Q_{+}} \operatorname{Irr} \Lambda(\nu) ; \mathrm{wt}, \varepsilon_{i}^{*}, \varphi_{i}^{*}, \widetilde{e}_{i}^{*}, \widetilde{f}_{i}^{*}\right)$ is a crystal and the bijection $\Phi$ gives an isomorphism of crystals form $\left(B(\infty) ; \mathrm{wt}, \varepsilon_{i}^{*}, \varphi_{i}^{*}, \widetilde{e}_{i}^{*}, \widetilde{f}_{i}^{*}\right)$ to it.

Remark . Because of (7.1.1), the definition of the map $\Phi: B(\infty) \stackrel{\sim}{\rightarrow} \bigsqcup_{\nu \in Q_{+}} \operatorname{Irr} \Lambda(\nu)$ is independent of the choice of $\mathbf{i}$.

7.3. A proof of Lemma 5.6.5. We only show the second formula:

$$
\tilde{f}_{i}^{*}(\mathbf{M}(\mathbf{a}))=\mathbf{M}\left(\tilde{f}_{i}^{*} \mathbf{a}\right) \quad(\mathbf{a} \in \mathcal{B}),
$$

because the first one is proved by similar method.

By Corollary 5.4.1, it is enough to show that

$$
\begin{gathered}
M_{[1, i]^{c}}\left(\tilde{f}_{i}^{*} \mathbf{a}\right)=M_{[1, i]^{c}}(\mathbf{a})-1, \\
M_{K}\left(\widetilde{f}_{i}^{*} \mathbf{a}\right)=M_{K}(\mathbf{a}) \text { for all } K \in \mathcal{M}_{n}^{\times} \backslash \mathcal{M}_{n}^{\times}(i)^{*} .
\end{gathered}
$$

It is easy to see (7.3.1). Indeed, as in the proof of Lemma 5.6.4, we have

$$
\operatorname{wt}(\mathbf{a})=\operatorname{wt}(\mathbf{M}(\mathbf{a}))=\sum_{i \in I} M_{[1, i]^{c}}(\mathbf{a}) \alpha_{i} .
$$

Similarly we have

$$
\mathrm{wt}\left(\tilde{f}_{i}^{*} \mathbf{a}\right)=\sum_{i \in I} M_{[1, i]^{c}}\left(\tilde{f}_{i}^{*} \mathbf{a}\right) \alpha_{i} .
$$

Since $\mathcal{B}$ is a $*$-crystal, we have $\operatorname{wt}\left(\tilde{f}_{i}^{*} \mathbf{a}\right)=\operatorname{wt}(\mathbf{a})-\alpha_{i}$. Therefore (7.3.1) holds.

We shall prove (7.3.2). Assume $K \in \mathcal{M}_{n}^{\times} \backslash \mathcal{M}_{n}^{\times}(i)^{*}$. Namely, $i \in K$ or $i+1 \notin K$. By (7.1.2) and Theorem 7.2.1, it is enough to prove that $M_{K}\left(\widetilde{f}_{i}^{*} \Lambda_{\mathbf{a}}\right)=M_{K}\left(\Lambda_{\mathbf{a}}\right)$ for $i \in K$ or $i+1 \notin K$. Moreover, since $\widetilde{e}_{i}^{* \max }\left(\widetilde{f}_{i}^{*} \Lambda_{\mathbf{a}}\right)=\widetilde{e}_{i}^{* \max } \Lambda_{\mathbf{a}}$, it is enough to show that

$$
M_{K}\left(\widetilde{e}_{i}^{* \max } \Lambda_{\mathbf{a}}\right)=M_{K}\left(\Lambda_{\mathbf{a}}\right) \quad(i \in K \text { or } i+1 \notin K) .
$$

Assume $i \in K_{m}=\left[s_{m}+1, t_{m}\right] \subset K$. Then, there are the following three cases; (a) $m=1$ and $s_{1}=1$, (b) $m=l$ and $t_{m}=n+1$, (c) otherwise. In the case (a), there is the path $\sigma\left(1 \leftarrow t_{1}\right)$ from $t_{1}$ to 1 in $\Omega(K)$ which is is trough $i$. Since 1 (the end point of this path) is not an element of in $(K)$, this path does not appear in the definition of $M_{K}(\Lambda)$ for any $\Lambda$. Therefore $M_{K}\left(\widetilde{e}_{i}^{* \max } \Lambda_{\mathbf{a}}\right)=M_{K}\left(\Lambda_{\mathbf{a}}\right)$. By the similar way, we have (7.3.3) in the case (b).

Let us consider the case (c). In this case, the path $\sigma\left(s_{m} \leftarrow t_{m}\right)$ in $\Omega(K)$ is trough $i$ and $s_{m} \in \operatorname{in}(K), t_{m} \in \operatorname{out}(K)$. We remark that $s_{m}<i$ since $i \in K$. For simplicity, we denote $\Lambda=\Lambda_{\mathbf{a}}$ and $\bar{\Lambda}=\widetilde{e}_{i}^{* \max } \Lambda_{\mathbf{a}}$. Let $\nu=\operatorname{wt}(\Lambda)$ and $\bar{\nu}=\operatorname{wt}(\bar{\Lambda})$, respectively. Take a general point $\bar{B}=\left(\bar{B}_{\tau}\right)_{\tau \in H} \in \bar{\Lambda}$. Recall the diagram (7.2.3) and take a general point $B=\left(B_{\tau}\right)_{\tau \in H} \in \Lambda(\nu)$ of $q_{2} \circ q_{1}^{-1}(B)$. Then $B$ is a general point of $\Lambda$. By the constriction we have the following commutative diagram:

$$
\begin{aligned}
& \bar{\phi}_{t_{m}} \\
& V_{t_{m}}(\nu) \stackrel{\sim}{\rightarrow} V_{t_{m}}(\bar{\nu}) \\
& B_{\sigma\left(i \leftarrow t_{m}\right)} \downarrow \quad \bar{\phi}_{i} \quad \downarrow \bar{B}_{\sigma\left(i \leftarrow t_{m}\right)} \\
& V_{i}(\nu) \rightarrow V_{i}(\bar{\nu}) \\
& B_{\sigma\left(s_{m} \leftarrow i\right)} \downarrow \quad \bar{\phi}_{s_{m}} \downarrow \bar{B}_{\sigma\left(s_{m} \leftarrow i\right)} \\
& V_{s_{m}}(\nu) \stackrel{\sim}{\rightarrow} V_{s_{m}}(\bar{\nu}) \text {. }
\end{aligned}
$$


Therefore we have

$$
\operatorname{Im}\left(B_{\sigma\left(s_{m} \leftarrow t_{m}\right)}\right)=\operatorname{Im}\left(\bar{B}_{\sigma\left(s_{m} \leftarrow t_{m}\right)}\right)
$$

and this formula tells us (7.3.3) holds.

For the case of $i+1 \notin K$, we have (7.3.3) by the similar method. Thus we complete a proof of Lemma 5.6.5.

\section{A NeW Proof of the Anderson-Mirković COnjecture}

8.1. Reformulation of the Anderson-Mirković conjecture. Let us denote $\Lambda=\Lambda_{\mathbf{a}}$. Then Corollary 5.5 .2 can be written as

$$
\left(\widetilde{f}_{i}^{*} \mathbf{M}(\Lambda)\right)_{K}= \begin{cases}\min \left\{M_{K}(\Lambda), M_{s_{i} K}(\Lambda)+c_{i}^{*}(\mathbf{M}(\Lambda))\right\} & \left(K \in \mathcal{M}_{n}^{\times}(i)^{*}\right), \\ M_{K}(\Lambda) & \text { (otherwise) } .\end{cases}
$$

Here $c_{i}^{*}(\mathbf{M}(\Lambda))=M_{[1, i]^{c}}(\Lambda)-M_{([1, i+1] \backslash\{i\})^{c}}(\Lambda)-1$. By Lemma 5.6.5, we already know that

$$
\left(\tilde{f}_{i}^{*} \mathbf{M}(\Lambda)\right)_{K}=M_{K}\left(\tilde{f}_{i}^{*} \Lambda\right) \quad \text { for } K \in \mathcal{M}_{n}^{\times}
$$

Moreover, by (7.3.2), we have

$$
\left(\tilde{f}_{i}^{*} \mathbf{M}(\Lambda)\right)_{K}=M_{K}(\Lambda) \quad \text { for } K \in \mathcal{M}_{n}^{\times} \backslash \mathcal{M}_{n}^{\times}(i)^{*} .
$$

Therefore it is enough to show

$$
M_{K}\left(\widetilde{f}_{i}^{*} \Lambda\right)=\min \left\{M_{K}(\Lambda), M_{s_{i} K}(\Lambda)+c_{i}^{*}(\mathbf{M}(\Lambda))\right\} \text { for } K \in \mathcal{M}_{n}^{\times}(i)^{*} .
$$

Lemma 8.1.1. The formula (8.1.1) is equivalent to the following:

$$
M_{K}(\Lambda)=\min \left\{M_{K}(\bar{\Lambda}), M_{s_{i} K}(\bar{\Lambda})+\left\langle h_{i}, \operatorname{wt}(\bar{\Lambda})\right\rangle-\varepsilon_{i}^{*}(\Lambda)\right\} \text { for } K \in \mathcal{M}_{n}^{\times}(i)^{*} .
$$

Here $\bar{\Lambda}=\widetilde{e}_{i}^{* \max } \Lambda$.

Remark . The formula (8.1.2) is a generalization of the formula which appears in our previous paper [KS].

Consider the case of $K=[1, i+1] \backslash\{i\}(1 \leq i \leq n)$. Then we have

$$
\operatorname{out}(K)=\left\{\begin{array}{ll}
\{2\} & (i=1), \\
\{i-1, i+1\} & (2 \leq i \leq n-1), \\
\{n-1\} & (i=n)
\end{array} \text { and } \operatorname{in}(K)=\{i\} .\right.
$$

Therefore we have

$$
\begin{aligned}
M_{[1, i+1] \backslash\{i\}}(\Lambda) & =-\operatorname{dim}_{\mathbb{C}} \operatorname{Coker}\left(\underset{\tau ; \operatorname{in}(\tau)=i}{\oplus} V(\nu)_{\text {out }(\tau)} \stackrel{\oplus B_{\mathcal{T}}}{\longrightarrow} V(\nu)_{i}\right) \\
& =-\varepsilon_{i}(\Lambda) .
\end{aligned}
$$

Here $B=\left(B_{\tau}\right)$ is a general point of $\Lambda$. Since $s_{i} K=[1, i]$, the formula (8.1.2) is equivalent to

$$
\varepsilon_{i}(\Lambda)=\max \left\{\varepsilon_{i}(\bar{\Lambda}),-\left\langle h_{i}, \operatorname{wt}(\bar{\Lambda})\right\rangle+\varepsilon_{i}^{*}(\Lambda)\right\} .
$$

This is nothing but the formula which appears in [KS], Proposition 5.3.1, (1).

Proof of Lemma 8.1.1. Let $\nu, \bar{\nu}, B, \bar{B}$ be as same as in the proof of Lemma 5.6.5. For simplicity, we denote $\oplus_{i \in I} V_{i}=\oplus_{i \in I} V_{i}(\nu)$ and $\oplus_{i \in I} \bar{V}_{i}=\oplus_{i \in I} V_{i}(\bar{\nu})$.

Before proving the equivalence, we shall show

$$
c_{i}^{*}(\mathbf{M}(\Lambda))=\left\langle h_{i}, \operatorname{wt}(\bar{\Lambda})\right\rangle-\varepsilon_{i}^{*}(\Lambda)-1 .
$$

Since

$$
M_{[1, i]^{c}}(\Lambda)=-\operatorname{dim}_{\mathbb{C}} V_{i},
$$


we have

$$
M_{([1, i+1] \backslash\{i\})^{c}}(\Lambda)=-\operatorname{dim}_{\mathbb{C}} \operatorname{Coker}\left(V_{i} \rightarrow \underset{\tau ; \operatorname{out}(\tau)=i}{\oplus} V_{\operatorname{in}(\tau)}\right),
$$

$$
\begin{aligned}
M_{[1, i]^{c}} & (\Lambda)-M_{([1, i+1] \backslash\{i\})^{c}}(\Lambda) \\
& =-\operatorname{dim}_{\mathbb{C}} V_{i}+\operatorname{dim}_{\mathbb{C}} \operatorname{Coker}\left(V_{i} \rightarrow \underset{\tau ; \operatorname{out}(\tau)=i}{\oplus} V_{\operatorname{in}(\tau)}\right) \\
& =-2 \operatorname{dim}_{\mathbb{C}} V_{i}+\operatorname{dim}_{\mathbb{C}}\left(\underset{\tau ; \operatorname{out}(\tau)=i}{\oplus} V_{\operatorname{in}(\tau)}\right)+\operatorname{dim}_{\mathbb{C}} \operatorname{Ker}\left(V_{i} \rightarrow \underset{\tau ; \operatorname{out}(\tau)=i}{\oplus} V_{\operatorname{in}(\tau)}\right) \\
& =\left\langle h_{i}, \operatorname{wt}(\Lambda)\right\rangle+\varepsilon_{i}^{*}(\Lambda) .
\end{aligned}
$$

Moreover, since

we have

$$
\operatorname{dim}_{\mathbb{C}} \bar{V}_{k}= \begin{cases}\operatorname{dim}_{\mathbb{C}} V_{k} & (k \neq i) \\ \operatorname{dim}_{\mathbb{C}} V_{i}-\varepsilon_{i}^{*}(\Lambda) & (k=i)\end{cases}
$$

$$
\begin{aligned}
c_{i}^{*}(\mathbf{M}(\Lambda)) & =\left\langle h_{i}, \operatorname{wt}(\Lambda)\right\rangle+\varepsilon_{i}^{*}(\Lambda)-1 \\
& =\left\langle h_{i}, \operatorname{wt}(\bar{\Lambda})-\varepsilon_{i}^{*}(\Lambda) \alpha_{i}\right\rangle+\varepsilon_{i}^{*}(\Lambda)-1 \\
& =\left\langle h_{i}, \operatorname{wt}(\bar{\Lambda})\right\rangle-\varepsilon_{i}^{*}(\Lambda)-1 .
\end{aligned}
$$

Thus, (8.1.3) is proved.

Let us prove the equivalence. Firstly, we will show (8.1.1) $\Rightarrow$ (8.1.2). Applying (8.1.1) for $\Lambda_{1}=\widetilde{e}_{i}^{*} \Lambda$, we have

$$
\begin{aligned}
M_{K}(\Lambda) & =M_{K}\left(\tilde{f}_{i}^{*} \Lambda_{1}\right) \\
& =\min \left\{M_{K}\left(\Lambda_{1}\right), M_{s_{i} K}\left(\Lambda_{1}\right)+c_{i}^{*}\left(\mathbf{M}\left(\Lambda_{1}\right)\right)\right\} .
\end{aligned}
$$

Since the vertex $i$ is a source in $\Omega\left(s_{i} K\right)$ and $\overline{\Lambda_{1}}=\bar{\Lambda}$, we have

$$
M_{s_{i} K}\left(\Lambda_{1}\right)=M_{s_{i} K}\left(\overline{\Lambda_{1}}\right)=M_{s_{i} K}(\bar{\Lambda}) \text {. }
$$

On the other hand,

$$
\begin{aligned}
c_{i}^{*}\left(\mathbf{M}\left(\Lambda_{1}\right)\right) & =\left\langle h_{i}, \operatorname{wt}\left(\overline{\Lambda_{1}}\right)\right\rangle-\varepsilon_{i}^{*}\left(\Lambda_{1}\right)-1 \\
& =\left\langle h_{i}, \operatorname{wt}(\bar{\Lambda})\right\rangle-\varepsilon_{i}^{*}(\Lambda) .
\end{aligned}
$$

Therefore we have

$$
M_{K}(\Lambda)=\min \left\{M_{K}\left(\Lambda_{1}\right), M_{s_{i} K}(\bar{\Lambda})+\left\langle h_{i}, \operatorname{wt}(\bar{\Lambda})\right\rangle-\varepsilon_{i}^{*}(\Lambda)\right\} .
$$

Similarly, applying (8.1.1) for $\Lambda_{2}=\widetilde{e}_{i}^{*} \Lambda_{1}=\left(\widetilde{e}_{i}^{*}\right)^{2} \Lambda$, we have

$$
\begin{aligned}
M_{K}\left(\Lambda_{1}\right) & =\min \left\{M_{K}\left(\Lambda_{2}\right), M_{s_{i} K}(\bar{\Lambda})+\left\langle h_{i}, \operatorname{wt}(\bar{\Lambda})\right\rangle-\varepsilon_{i}^{*}\left(\Lambda_{1}\right)\right\} \\
& =\min \left\{M_{K}\left(\Lambda_{2}\right), M_{s_{i} K}(\bar{\Lambda})+\left\langle h_{i}, \operatorname{wt}(\bar{\Lambda})\right\rangle-\varepsilon_{i}^{*}(\Lambda)+1\right\} .
\end{aligned}
$$

By substituting this formula for (8.1.4), we have

$$
\begin{gathered}
M_{K}(\Lambda)=\min \left\{\min \left\{M_{K}\left(\Lambda_{2}\right), M_{s_{i} K}(\bar{\Lambda})+\left\langle h_{i}, \operatorname{wt}(\bar{\Lambda})\right\rangle-\varepsilon_{i}^{*}(\Lambda)+1\right\},\right. \\
\left.M_{s_{i} K}(\bar{\Lambda})+\left\langle h_{i}, \operatorname{wt}(\bar{\Lambda})\right\rangle-\varepsilon_{i}^{*}(\Lambda)\right\} \\
=\min \left\{M_{K}\left(\Lambda_{2}\right), M_{s_{i} K}(\bar{\Lambda})+\left\langle h_{i}, \operatorname{wt}(\bar{\Lambda})\right\rangle-\varepsilon_{i}^{*}(\Lambda)\right\} .
\end{gathered}
$$

After repeating the similar method, we have

$$
M_{K}(\Lambda)=\min \left\{M_{K}(\bar{\Lambda}), M_{s_{i} K}(\bar{\Lambda})+\left\langle\alpha_{i}, \operatorname{wt}(\bar{\Lambda})\right\rangle-\varepsilon_{i}^{*}(\Lambda)\right\} .
$$


This is nothing but the formula (8.1.2).

Secondly let us prove $(8.1 .2) \Rightarrow(8.1 .1)$. By (8.1.2) for $\widetilde{f}_{i}^{*} \Lambda$ and $\overline{\tilde{f}_{i}^{*} \Lambda}=\bar{\Lambda}$, we have

$$
\begin{aligned}
M_{K}\left(\widetilde{f}_{i}^{*} \Lambda\right) & =\min \left\{M_{K}\left(\overline{\tilde{f}_{i}^{*} \Lambda}\right), M_{s_{i} K}\left(\overline{\tilde{f}_{i}^{*} \Lambda}\right)+\left\langle\alpha_{i}, \operatorname{wt}\left(\overline{\tilde{f}_{i}^{*} \Lambda}\right)\right\rangle-\varepsilon_{i}^{*}\left(\tilde{f}_{i}^{*} \Lambda\right)\right\} \\
& =\min \left\{M_{K}(\bar{\Lambda}), M_{s_{i} K}(\bar{\Lambda})+\left\langle h_{i}, \operatorname{wt}(\bar{\Lambda})\right\rangle-\varepsilon_{i}^{*}(\Lambda)-1\right\} .
\end{aligned}
$$

Since $M_{s_{i} K}(\bar{\Lambda})+\left\langle h_{i}, \operatorname{wt}(\bar{\Lambda})\right\rangle-\varepsilon_{i}^{*}(\Lambda)>M_{s_{i} K}(\bar{\Lambda})+\left\langle h_{i}, \operatorname{wt}(\bar{\Lambda})\right\rangle-\varepsilon_{i}^{*}(\Lambda)-1$,

the right hand side $=\min \left\{\min \left\{M_{K}(\bar{\Lambda}), M_{s_{i} K}(\bar{\Lambda})+\left\langle h_{i}, \operatorname{wt}(\bar{\Lambda})\right\rangle-\varepsilon_{i}^{*}(\Lambda)\right\}\right.$,

$$
\begin{aligned}
& \left.M_{s_{i} K}(\bar{\Lambda})+\left\langle h_{i}, \operatorname{wt}(\bar{\Lambda})\right\rangle-\varepsilon_{i}^{*}(\Lambda)-1\right\} \\
= & \min \left\{M_{K}(\Lambda), M_{s_{i} K}(\bar{\Lambda})+\left\langle h_{i}, \operatorname{wt}(\bar{\Lambda})\right\rangle-\varepsilon_{i}^{*}(\Lambda)-1\right\} \\
= & \min \left\{M_{K}(\Lambda), M_{s_{i} K}(\bar{\Lambda})+c_{i}^{*}(\mathbf{M}(\Lambda))\right\} .
\end{aligned}
$$

Because $s_{i} K \in \mathcal{M}_{n}^{\times} \backslash \mathcal{M}_{n}^{\times}(i)^{*}$, we have $M_{s_{i} K}(\bar{\Lambda})=M_{s_{i} K}(\Lambda)$ by (7.3.3). Therefore we have

$$
M_{K}\left(\widetilde{f}_{i}^{*} \Lambda\right)=\min \left\{M_{K}(\Lambda), M_{s_{i} K}(\Lambda)+c_{i}^{*}(\mathbf{M}(\Lambda))\right\} .
$$

This is nothing but (8.1.1).

8.2. A proof of the formula (8.1.2). The aim of this subsection is to prove the next proposition:

Proposition 8.2.1. The formula (8.1.2) holds for any $K \in \mathcal{M}_{n}^{\times}(i)^{*}$.

Set

$$
\bar{W}_{s_{i} K}=\operatorname{Ker}\left(\underset{p \in \operatorname{out}\left(s_{i} K\right)}{\oplus} \bar{V}_{p} \stackrel{\oplus \bar{B}_{p \rightarrow q}}{\longrightarrow} \underset{q \in \operatorname{in}\left(s_{i} K\right)}{\oplus} \bar{V}_{q}\right) \subset\left(\underset{p \in \operatorname{out}\left(s_{i} K\right)}{\oplus} \bar{V}_{p}\right) .
$$

By the assumption, we have

$$
\operatorname{out}\left(s_{i} K\right) \backslash\{i\} \subset \operatorname{out}(K) \subset\left(\operatorname{out}\left(s_{i} K\right) \backslash\{i\}\right) \cup\{i-1, i+1\} .
$$

Therefore we can define a map $\Phi: \bar{W}_{s_{i} K} \rightarrow\left(\oplus_{k \in \operatorname{out}(K)} V_{k}\right)$ by

$$
\Phi\left(\sum_{p \in \operatorname{Out}\left(s_{i} K\right)} \bar{w}_{p}\right)=\delta_{K}(i-1) \bar{B}_{i \rightarrow i-1}\left(\bar{w}_{i}\right)+\delta_{K}(i+1) \bar{B}_{i \rightarrow i+1}\left(\bar{w}_{i}\right)+\sum_{p \in \operatorname{Out}\left(s_{i} K\right) \backslash\{i\}} \bar{w}_{p},
$$

where $\bar{w}_{p} \in \bar{V}_{p}$ and $\delta_{K}$ is a map form $[1, n]$ to $\{0,1\}$ defined by

$$
\delta_{K}(k)= \begin{cases}1 & (k \in \operatorname{out}(K)) \\ 0 & \text { (otherwise) }\end{cases}
$$

Here we remark that, if $p \in \operatorname{out}\left(s_{i} K\right) \backslash\{i\}$, we have $p \in \operatorname{out}(K)$ and $\bar{V}_{p}=V_{p}$.

Set

$$
N=\operatorname{Coker}(\Phi)
$$

and consider a map

$$
\widetilde{\mathrm{Id}}:\left(\underset{k \in \operatorname{out}(K)}{\oplus} V_{k}\right) \rightarrow N
$$


which is naturally induced form the identity map Id : $\left(\underset{k \in \operatorname{out}(K)}{\oplus} V_{k}\right) \stackrel{\sim}{\rightarrow}\left(\underset{k \in \operatorname{out}(K)}{\oplus} V_{k}\right)$.

By the construction, it is clear that $\widetilde{\text { Id }}$ is surjective.

In the above setting, the following two Lemmas hold:

Lemma 8.2.2. $\Phi$ is injective.

Lemma 8.2.3. Let $k \in \operatorname{out}(K), l \in \operatorname{in}(K)$ and assume that there is a path $\sigma(k \rightarrow l)$ in the orientation $\Omega(K)$.

(1) If $l \neq i$, then the map $B_{\sigma(k \rightarrow l)}: V_{k} \rightarrow V_{l}\left(=\bar{V}_{l}\right)$ induces a map $\psi_{l}: N \rightarrow V_{l}$ such that

$$
B_{\sigma(k \rightarrow l)}\left(=\bar{B}_{\sigma(k \rightarrow l)}\right)=\psi_{l} \circ \widetilde{I} d .
$$

(2) If $l=i$, then the map $B_{\sigma(k \rightarrow i)}: V_{k} \rightarrow \bar{V}_{i}\left(\neq V_{i}\right)$ induces a map $\psi_{i}: N \rightarrow \bar{V}_{i}$ such that

$$
\bar{B}_{\sigma(k \rightarrow i)}=\psi_{i} \circ \widetilde{I} d \text {. }
$$

Moreover, let $\pi_{i}: V_{i} \rightarrow \bar{V}_{i}$ be the natural projection and $\varphi_{i}: N \rightarrow V_{i}$ a generic map such that $\psi_{i}=\pi_{i} \circ \varphi_{i}$. Then we have

$$
B_{\sigma(k \rightarrow i)}=\varphi_{i} \circ \widetilde{I} d .
$$

The above two lemmas are easy exercises on linear algebra. So, we omit to give proofs.

Proof of Proposition 8.2.1. Since $\Phi$ is injective, we have

$$
\begin{aligned}
& \operatorname{dim}_{\mathbb{C}} N=\sum_{k \in \operatorname{out}(K)} \operatorname{dim}_{\mathbb{C}} V_{k}-\operatorname{dim}_{\mathbb{C}} \bar{W}_{s_{i} K} \\
& =\sum_{k \in \operatorname{out}(K)} \operatorname{dim}_{\mathbb{C}} V_{k}-\operatorname{dim}_{\mathbb{C}} \operatorname{Ker}\left(\underset{p \in \operatorname{out}\left(s_{i} K\right)}{\oplus} \bar{V}_{p} \stackrel{\oplus \bar{B}_{\sigma(p \rightarrow q)}}{\longrightarrow} \underset{q \in \operatorname{in}\left(s_{i} K\right)}{\oplus} \bar{V}_{q}\right) \\
& =\sum_{k \in \operatorname{out}(K)} \operatorname{dim}_{\mathbb{C}} V_{k}-\sum_{p \in \operatorname{out}\left(s_{i} K\right)} \operatorname{dim}_{\mathbb{C}} \bar{V}_{p}+\sum_{q \in \operatorname{in}\left(s_{i} K\right)} \operatorname{dim}_{\mathbb{C}} \bar{V}_{q} \\
& -\operatorname{dim}_{\mathbb{C}} \operatorname{Coker}\left(\underset{p \in \operatorname{out}\left(s_{i} K\right)}{\oplus} \bar{V}_{p} \stackrel{\oplus \bar{B}_{\sigma(p \rightarrow q)}}{\longrightarrow} \underset{q \in \operatorname{in}\left(s_{i} K\right)}{\oplus} \bar{V}_{q}\right) \\
& =\sum_{k \in \operatorname{out}(K)} \operatorname{dim}_{\mathbb{C}} V_{k}-\sum_{p \in \operatorname{out}\left(s_{i} K\right)} \operatorname{dim}_{\mathbb{C}} \bar{V}_{p}+\sum_{q \in \operatorname{in}\left(s_{i} K\right)} \operatorname{dim}_{\mathbb{C}} \bar{V}_{q}+M_{s_{i} K}(\bar{\Lambda}) .
\end{aligned}
$$

Denote the direct sum of the maps $\psi_{l}\left(\right.$ resp. $\left.\varphi_{l}\right)(l \in$ in $(\mathrm{K}))$ by

$$
\psi=\underset{l \in \operatorname{in}(K)}{\oplus} \psi_{l}: N \rightarrow \underset{l \in \operatorname{in}(K)}{\oplus} \bar{V}_{l} \quad\left(\text { resp. } \varphi=\underset{l \in \operatorname{in}(K)}{\oplus} \varphi_{l}: N \rightarrow \underset{l \in \operatorname{in}(K)}{\oplus} V_{l}\right) .
$$

Here we set $\varphi_{l}=\psi_{l}$ for $l \neq i$.

By the definition, we have

$$
\operatorname{Im}\left(\underset{k \in \operatorname{out}(K)}{\oplus} V_{k} \stackrel{\oplus B_{\sigma(k \rightarrow l)}}{\longrightarrow} \underset{l \in \operatorname{in}(K)}{\oplus} V_{l}\right)=\operatorname{Im}\left(N \stackrel{\varphi}{\longrightarrow} \underset{l \in \operatorname{in}(K)}{\oplus} V_{l}\right) .
$$

Moreover, by the genericity of $\varphi$, we have

$$
\operatorname{dim} \operatorname{Ker} \varphi=\max \left\{\operatorname{dim}_{\mathbb{C}} \operatorname{Ker} \psi-\varepsilon_{i}^{*}(\Lambda), 0\right\} .
$$


Combining the above results, we have

$$
-M_{K}(\Lambda)=\sum_{l \in \operatorname{in}(K)} \operatorname{dim}_{\mathbb{C}} V_{l}-\operatorname{dim}_{\mathbb{C}} N+\max \left\{\operatorname{dim}_{\mathbb{C}} \operatorname{Ker} \psi-\varepsilon_{i}^{*}(\Lambda), 0\right\} .
$$

Indeed,

$$
\begin{aligned}
-M_{K}(\Lambda) & =\operatorname{dim}_{\mathbb{C}} \operatorname{Coker}\left(\underset{k \in \operatorname{out}(K)}{\oplus} V_{k} \stackrel{\oplus B_{\sigma(k \rightarrow l)}}{\longrightarrow} \underset{l \in \operatorname{in}(K)}{\oplus} V_{l}\right) \\
& =\sum_{l \in \operatorname{in}(K)} \operatorname{dim}_{\mathbb{C}} V_{l}-\operatorname{dim}_{\mathbb{C}} \operatorname{Im}\left(\underset{k \in \operatorname{out}(K)}{\oplus} V_{k} \stackrel{\left.\oplus B_{\sigma(k \rightarrow l)} \underset{l \in \operatorname{in}(K)}{\oplus} V_{l}\right)}{\longrightarrow} \sum_{l \in \operatorname{in}(K)}^{\oplus} \operatorname{dim}_{\mathbb{C}} V_{l}-\operatorname{dim}_{\mathbb{C}} \operatorname{Im} \varphi\right. \\
& =\sum_{l \in \operatorname{in}(K)} \operatorname{dim}_{\mathbb{C}} V_{l}-\operatorname{dim}_{\mathbb{C}} N+\operatorname{dim}_{\mathbb{C}} \operatorname{Ker} \varphi \\
& =\sum_{l \in \operatorname{in}(K)} \operatorname{dim}_{\mathbb{C}} V_{l}-\operatorname{dim}_{\mathbb{C}} N+\max \left\{\operatorname{dim}_{\mathbb{C}} \operatorname{Ker} \psi-\varepsilon_{i}^{*}(\Lambda), 0\right\} .
\end{aligned}
$$

Lemma 8.2.4. The following formulas hold:

(1) $\sum_{l \in \operatorname{in}(K)} \operatorname{dim}_{\mathbb{C}} V_{l}-\operatorname{dim}_{\mathbb{C}} N+\operatorname{dim}_{\mathbb{C}} \operatorname{Ker} \psi-\varepsilon_{i}^{*}(\Lambda)=-M_{K}(\bar{\Lambda})$,

(2) $\sum_{l \in \operatorname{in}(K)} \operatorname{dim}_{\mathbb{C}} V_{l}-\operatorname{dim}_{\mathbb{C}} N=-M_{s_{i} K}(\bar{\Lambda})+\varepsilon_{i}^{*}(\Lambda)-\left\langle h_{i}, \operatorname{wt}(\bar{\Lambda})\right\rangle$.

Proof. The formula (1) is proved by a direct computation. Indeed, we have

$$
\begin{aligned}
\sum_{l \in \operatorname{in}(K)} \operatorname{dim}_{\mathbb{C}} V_{l}-\operatorname{dim}_{\mathbb{C}} N+\operatorname{dim}_{\mathbb{C}} \operatorname{Ker} \psi-\varepsilon_{i}^{*}(\Lambda) & \\
& =\sum_{l \in \operatorname{in}(K)} \operatorname{dim}_{\mathbb{C}} \bar{V}_{l}-\operatorname{dim}_{\mathbb{C}} \operatorname{Im} \psi \\
& =\sum_{l \in \operatorname{in}(K)} \operatorname{dim}_{\mathbb{C}} \bar{V}_{l}-\operatorname{dim}_{\mathbb{C}} \operatorname{Im}\left(\underset{k \in \operatorname{out}(K)}{\oplus} \bar{V}_{k} \stackrel{\left.\oplus \bar{B}_{\sigma(k \rightarrow l)} \underset{l \in \operatorname{in}(K)}{\oplus} \bar{V}_{l}\right)}{\longrightarrow}\right. \\
& =\operatorname{dim}_{\mathbb{C}} \operatorname{Coker}\left(\underset{k \in \operatorname{out}(K)}{\oplus} \bar{V}_{k} \stackrel{\left.\oplus \bar{B}_{\sigma(k \rightarrow l)} \underset{l \in \operatorname{in}(K)}{\oplus} \bar{V}_{l}\right)}{=}\right. \\
& =-M_{K}(\bar{\Lambda}) .
\end{aligned}
$$

Let us show the formula (2). We have

$$
\begin{aligned}
\sum_{l \in \operatorname{in}(K)} \operatorname{dim}_{\mathbb{C}} V_{l}-\operatorname{dim}_{\mathbb{C}} N=\sum_{l \in \operatorname{in}(K)} \operatorname{dim}_{\mathbb{C}} V_{l}-\sum_{k \in \operatorname{out}(K)} \operatorname{dim}_{\mathbb{C}} V_{k} \\
+\sum_{p \in \operatorname{out}\left(s_{i} K\right)} \operatorname{dim}_{\mathbb{C}} \bar{V}_{p}-\sum_{q \in \operatorname{in}\left(s_{i} K\right)} \operatorname{dim}_{\mathbb{C}} \bar{V}_{q}-M_{s_{i} K}(\bar{\Lambda}) .
\end{aligned}
$$


Therefore it is enough to show that

$$
\begin{gathered}
\sum_{l \in \operatorname{in}(K)} \operatorname{dim} V_{l}-\sum_{k \in \operatorname{out}(K)} \operatorname{dim} V_{k}+\sum_{p \in \operatorname{out}\left(s_{i} K\right)} \operatorname{dim} \bar{V}_{p}-\sum_{q \in \operatorname{in}\left(s_{i} K\right)} \operatorname{dim} \bar{V}_{q} \\
=\varepsilon_{i}^{*}(\Lambda)-\left\langle h_{i}, \operatorname{wt}(\bar{\Lambda})\right\rangle .
\end{gathered}
$$

But it is easily checked by a direct computation. Thus, we get the formula.

Let us return to the proof of Proposition 8.2.1. Substituting the result of the above lemma for (8.2.1), we get

$$
-M_{K}(\Lambda)=\max \left\{-M_{K}(\bar{\Lambda}),-M_{s_{i} K}(\bar{\Lambda})-\left\langle\alpha_{i}, \operatorname{wt}(\bar{\Lambda})\right\rangle+\varepsilon_{i}^{*}(\Lambda)\right\} .
$$

This is nothing but the formula (8.1.2). Thus we have the statement.

\section{Appendix A. A proof of the formula (7.1.1)}

In this appendix, we will give a proof of the formula (7.1.1).

A.1. Results in [KS]. Let us recall some results in [KS]. Consider two quivers of type $A_{n},(I, \Omega)$ and $\left(I, \Omega^{\prime}\right)$. Assume $\mathbf{i}\left(\right.$ resp. $\left.\mathbf{i}^{\prime}\right)$ is a reduced word of the longest element which is adapted to the orientation $\Omega\left(\right.$ resp. $\left.\Omega^{\prime}\right)$.

Let $\nu \in Q_{+}$and $\mathcal{O}_{\mathbf{a}, \Omega}$ be the $G(\nu)$-orbit in $E_{V(\nu), \Omega}$ corresponding to an i-Lusztig datum $\mathbf{a}$, and $\mathcal{O}_{\mathbf{a}^{\prime}, \Omega^{\prime}}$ the orbit in $E_{V(\nu), \Omega^{\prime}}$ corresponding to an $\mathbf{i}^{\prime}$-Lusztig datum $\mathbf{a}^{\prime}=R_{\mathbf{i}}^{\mathbf{i}^{\prime}}(\mathbf{a})$ where $R_{\mathbf{i}}^{\mathbf{i}^{\prime}}$ is the transition map from $\mathbf{i}$ to $\mathbf{i}^{\prime}$.

Set

$$
\Lambda_{\mathbf{a}}:=\overline{T_{\mathcal{O}_{\mathbf{a}}, \Omega}^{*} E_{V(\nu), \Omega}} \quad \text { and } \quad \Lambda_{\mathbf{a}^{\prime}}:=\overline{T_{\mathcal{O}_{\mathbf{a}^{\prime}}, \Omega^{\prime}}^{*} E_{V(\nu), \Omega^{\prime}}} .
$$

The goal is to prove

$$
\Lambda_{\mathbf{a}}=\Lambda_{\mathbf{a}^{\prime}}
$$

Since our quiver is of type $A_{n}$, there uniquely exists a $G(\nu)$-orbit $\mathcal{O}_{\mathbf{b}, \Omega}$ in $E_{V(\nu), \Omega}$ such that $\Lambda_{\mathbf{a}^{\prime}}=\overline{T_{\mathcal{O}_{\mathbf{b}, \Omega}}^{*} E_{V(\nu), \Omega}}$. (Here we denote by $\mathbf{b}$ the corresponding $\mathbf{i}$-Lusztig datum.) We consider a map

$$
s:\left\{G(\nu) \text {-orbits in } E_{V(\nu), \Omega}\right\} \rightarrow\left\{G(\nu) \text {-orbits in } E_{V(\nu), \Omega}\right\}
$$

defined by $\mathcal{O}_{\mathbf{a}, \Omega} \mapsto \mathcal{O}_{\mathbf{b}, \Omega}$. We remark that, by the definition, $s$ is a bijection. To prove the formula (A.1.1) (or equivalently (7.1.1)), it is enough to show that $s$ is the identity map.

Let $\mathbb{C}_{\mathcal{O}_{\mathbf{a}, \Omega}}$ be the constant sheaf on the orbit $\mathcal{O}_{\mathbf{a}, \Omega},{ }^{\pi} \mathbb{C}_{\mathcal{O}_{\mathbf{a}, \Omega}}$ its minimal extension and $S S\left({ }^{\pi} \mathbb{C}_{\mathcal{O}_{\mathbf{a}, \Omega}}\right)$ its singular support. Then we have

$$
S S\left({ }^{\pi} \mathbb{C}_{\mathcal{O}_{\mathbf{a}, \Omega}}\right) \supset \Lambda_{\mathbf{a}}=\overline{T_{\mathcal{O}_{\mathbf{a}, \Omega}}^{*} E_{V(\nu), \Omega}} .
$$

In addition to the above, the next result is proved by Lusztig:

Theorem A.1.1 ([L1], [L2]).

$$
S S\left({ }^{\pi} \mathbb{C}_{\mathcal{O}_{\mathbf{a}, \Omega}}\right)=S S\left({ }^{\pi} \mathbb{C}_{\mathcal{O}_{\mathbf{a}^{\prime}, \Omega^{\prime}}}\right)
$$

Therefore we have

$$
S S\left({ }^{\pi} \mathbb{C}_{\mathcal{O}_{\mathbf{a}, \Omega}}\right)=S S\left({ }^{\pi} \mathbb{C}_{\mathcal{O}_{\mathbf{a}^{\prime}, \Omega^{\prime}}}\right) \supset \Lambda_{\mathbf{a}^{\prime}}=\overline{T_{\mathcal{O}_{\mathbf{b}, \Omega}}^{*} E_{V(\nu), \Omega}}=\overline{T_{s\left(\mathcal{O}_{\mathbf{a}, \Omega}\right)}^{*} E_{V(\nu), \Omega}}
$$


Remark . In [L2] and [KS], they consider a similar problem in more general setting. Let $\mathfrak{g}$ be an arbitrary symmetric Kac-Moody Lie algebra and $B(\infty)$ the crystal basis of $U_{q}^{-}(\mathfrak{g})$. In $[\mathrm{KS}]$, they define a crystal structure on the set of all irreducible components of Lusztig's quiver varieties, and show that it is isomorphic to $B(\infty)$. For $b \in B(\infty)$, we denote by $\Lambda_{b}$ the corresponding irreducible component. On the other hand, let $\mathbf{B}$ be the Lusztig's canonical basis of $U_{q}^{-}(\mathfrak{g})$. It is constructed as the set of certain simple perverse shaeves on the sepace of representations of a quiver attached to $\mathfrak{g}$ ([L2]). Recall that there is a canonical bijection between $B(\infty)$ and $\mathbf{B}$. For $b \in B(\infty)$, we denote by $L_{b, \Omega}$ the corresponding simple perverse sheaf. Then it is known that

$$
S S\left(L_{b, \Omega}\right) \supset \Lambda_{b} \quad \text { for any } b \in B(\infty) .
$$

Now, let us consider the following problem:

Problem. Assume $s: B(\infty) \rightarrow B(\infty)$ is a bijection such that $S S\left(L_{b, \Omega}\right) \supset \Lambda_{s(b)}$ for any $b \in B(\infty)$. Then, is $s$ the identity?

The above problem was firstly considered by Lusztig ([L2]). In [KS, they stated that "s must be the identity under the above assumption". But their "proof" of the statement is wrong. Therefore, the problem is still open for an arbitrary case.

On the other hand, Kimura $\mathrm{Kim}$ shows that the bijection $s$ must be the identity for finite $A D E$ type cases and cyclic quiver cases. In the next subsection, we will give a proof following $[\mathrm{Kim}]$.

A.2. Kimura's proof of (A.1.1). By $(A .1 .2)$ and the definition of singular supports, we have

$$
\overline{\mathcal{O}_{\mathbf{a}, \Omega}} \supset \mathcal{O}_{\mathbf{b}, \Omega}=s\left(\mathcal{O}_{\mathbf{a}, \Omega}\right) .
$$

Namely, the bijection $s$ preserves closure relations on $G(\nu)$-orbits in $E_{V(\nu), \Omega}$ :

$$
s\left(\mathcal{O}_{\Omega}\right) \subset \overline{\mathcal{O}_{\Omega}} \text { for any } G(\nu) \text { - orbit } \mathcal{O}_{\Omega} \text { in } E_{V(\nu), \Omega} .
$$

Hence, it is enough to prove that the following statement:

Proposition A.2.1 ([Kim $)$. Let $s$ be a bijection on the set of $G(\nu)$-orbits in $E_{V(\nu), \Omega}$ which preserves closure relations. Then s must be the identity.

Let us introduce an ordering $\leq$ on the set of $G(\nu)$-orbits in $E_{V(\nu), \Omega}$ by

$$
\mathcal{O}_{\Omega} \leq \mathcal{O}_{\Omega}^{\prime} \stackrel{\text { def }}{\Longleftrightarrow} \mathcal{O}_{\Omega}^{\prime} \subset \overline{\mathcal{O}_{\Omega}}
$$

By using this ordering, (A.2.1) can be rewritten as:

$$
s\left(\mathcal{O}_{\Omega}\right) \subset \overline{\mathcal{O}_{\Omega}}=\mathcal{O}_{\Omega} \cup \underset{\mathcal{O}_{\Omega}^{\prime}: \mathcal{O}_{\Omega}<\mathcal{O}_{\Omega}^{\prime}}{\bigcup} \mathcal{O}_{\Omega}^{\prime} .
$$

Proof of A.2.1. We will show the statement by the decreasing induction on $\leq$. Note that, by the definition, there is a maximal element with respect to the ordering $\leq$. Let $\mathcal{O}_{\Omega}$ be a such element. Hence, by $(A .2 .2)$ and the maximality of $\mathcal{O}_{\Omega}, s\left(\mathcal{O}_{\Omega}\right)$ must be equal to $\mathcal{O}_{\Omega}$. Assume $s\left(\mathcal{O}_{\Omega}^{\prime}\right)=\mathcal{O}_{\Omega}^{\prime}$ for any $\mathcal{O}_{\Omega}<\mathcal{O}_{\Omega}^{\prime}$. Since $s$ is a bijection, we have $s\left(\mathcal{O}_{\Omega}\right)=\mathcal{O}_{\Omega}$ by (A.2.2).

Remark . For finite $D, E$ cases and cyclic quiver cases, the similar method does work. Namely, we can show that $s$ must be the identity for such cases (see [Kim], in detail). 


\section{REFERENCES}

[A] J. E. Anderson, A polytope calculus for semisimple groups, Duke. Math. J. 116 (2003), 567-588.

[BFZ] A. Berenstein, S. Fomin and A. Zelevinsky, Parametrizations of canonical bases and totally positive matrices, Adv. Math. 122 (1996), 49-149.

[BG] A. Breverman and D. Gaitsgory, Crystals via the affine Grassmannian, Duke. Math. J. 107 (2001), 561-575.

[BK] P. Baumann and J. Kamnitzer, Preprojective algebras and MV polytopes, arXiv:1009.2469.

[EK] N. Enomoto and M. Kashiwara, Symmetric crystals for $\mathfrak{g} l_{\infty}$, Publ. RIMS. 44 (2008), 837-891.

[Kam1] J. Kamnitzer, Mirković-Vilonen cycles and polytopes, Ann. of Math. (2) 171 (2010), no. 1, 245-294.

[Kam2] J. Kamnitzer, The crystal structure on the set of Mirković-Vilonen polytopes, Adv. Math. 215 (2007), 66-93.

[KamS] J. Kamnitzer and C. Sadanand, Modules with 1-dimensional socle and components of Lusztig quiver varieties in type A, arXiv:1009.0272

[K1] M. Kashiwara, Crystallizing the q-analogue of universal enveloping algebras, Duke Math. J. 63 (1991), 465-516.

[K2] M. Kashiwara, Global crystal base of quantum groups, Duke Math. J. 69 (1993), 455-485.

[K3] M. Kashiwara, Crystal base and Littelmann's refined Demazure character formula, Duke Math. J. 71 (1993), 839-858.

[K4] M. Kashiwara, Bases cristallines des groupes quantiques, Cours Spécialisés Vol. 9, Société Mathématique de France, Paris, 2002.

[KS] M. Kashiwara and Y. Saito, Geometric construction of crystal bases, Duke Math. J. 89 (1997), 9-36.

[Kim] Y. Kimura, Affine quivers and crystal bases, Master thesis, Kyoto University (2007).

[Li] P. Littelmann, A Littlewood-Richardson rule for symmetrizable Kac-Moody Lie algebra, Invent. Math. 116 (1994), 329-346.

[L1] G. Lusztig, Canonical bases arising from quantized universal enveloping algebras, J. Amer. Math. Soc. 3 (1990), 447-498.

[L2] G. Lusztig, Quivers, perverse sheaves, and quantized universal enveloping algebras, J. Amer. Math. Soc. 4 (1991), 365-421.

[L3] G. Lusztig, Affine quivers and canonical bases, Publ. Math. IHES 76 (1992), 111-163.

[L4] G. Lusztig, Introduction to quantum groups, Progr. Math. 110 (1993), Birkhäuser.

[MV1] I. Mirković and K. Vilonen, Perverse sheaves on affine Grassmannian and Langlands duality, Math. Res. Lett. 7 (2000), 13-24.

[MV2] I. Mirković and K. Vilonen, Geometric Langlands duality and representations of algebraic groups over commutative rings, Ann. of Math. (2) 166 (2007), 95-143.

[NSS1] S. Naito, D. Sagaki and Y. Saito, Toward Berenstein-Zelevinsky data in affine type A, I: Construction of affine analogs, arXiv:1009.4526.

[NSS2] S. Naito, D.Sagaki and Y. Saito, Toward Berenstein-Zelevinsky data in affine type A, II: Explicit description, in preparation.

[NZ] T. Nakashima and A. Zelevinsky, Affine quivers and canonical bases, Publ. Math. IHES 76 (1992), 111-163.

[Re] M. Reineke, On the coloured graph structure of Lusztig's canonical basis, Math. Ann. 307 (1997), 705-723.

[S] Y. Saito, PBW basis of quantum universal enveloping algebras, Publ. RIMS. 30 (1994), 209-232.

[Sav] A. Savage, Geometric and combinatorial realization of crystal graphs, Algebr. Rep. Theory 9 (2006), 161-199.

Graduate School of Mathematical Sciences, University of Tokyo, 3-8-1 Komaba, MeguroKU, TOKYO 153-8914, JAPAN.

E-mail address: yosihisa@ms.u-tokyo.ac.jp 


\title{
MIRKOVIĆ-VILONEN POLYTOPES AND A QUIVER CONSTRUCTUION OF CRYSTAL BASIS IN TYPE $A$
}

\author{
YOSHIHISA SAITO
}

\begin{abstract}
In the current paper, we give a quiver theoretical interpretation of MirkovićVilonen polytopes in type $A_{n}$. As a by-product, we give a new proof of the AndersonMirković conjecture which describes the explicit forms of the actions of lowering Kashiwara operators on the set of Mirković-Vilonen polytopes.
\end{abstract}

\section{INTRODUCTION}

1.1. Let $B(\infty)$ be the crystal basis of the negative half of the quantum universal enveloping algebra associated with a symmetrizable Kac-Moody Lie algebra $\mathfrak{g}$. Each element $b \in B(\infty)$ can be written as

$$
b=\tilde{f}_{i_{1}} \tilde{f}_{i_{2}} \cdots \tilde{f}_{i_{k}} b_{\infty}
$$

Here $\tilde{f}_{i}(i \in I)$ is a lowering Kashiwara operator and $b_{\infty}$ the highest weight element of $B(\infty)$. However, for a given $b \in B(\infty)$, the above expression is not unique. For example, the following equality holds in the case of $\mathfrak{g}=\mathfrak{s} l_{3}$ :

$$
\widetilde{f}_{1}^{m} \widetilde{f}_{2}^{m+n} \widetilde{f}_{1}^{n} b_{\infty}=\widetilde{f}_{2}^{m} \widetilde{f}_{1}^{m+n} \widetilde{f}_{2}^{n} b_{\infty} \quad \text { (for any } m, n \in \mathbb{Z}_{\geq 0} \text { ). }
$$

Therefore, in the study of $B(\infty)$, it is important to give

- a parametrization of each element $b \in B(\infty)$ (we call it a realization of $B(\infty)$ ) and

- explicit identifications between several realizations.

Until now, many useful realizations of $B(\infty)$ are known. For example,

(a) a realization in terms of Lakshimibai-Seshadri path (Littelmann [Li]),

(b) a polyhedral realization (Nakashima and Zelevinsky [NZ]),

(c) a Lagrangian (or quiver) realization (Kashiwara and the author [KS]), etc.

These realizations work for arbitrary symmetrizable Kac-Moody Lie algebras. On the other hand, for the case that $\mathfrak{g}$ is a finite dimensional simple Lie algebras, there is

(d) a realization by using the theory of PBW basis (Lusztig [L1], the author [S]).

1.2. Recently, Kamnitzer [Kam1, Kam2] gave a new realization of $B(\infty)$ for the case that $\mathfrak{g}$ is a finite dimensional simple Lie algebras.

Let us explain a background of his work. In several years ago, Mirković and Vilonen introduced a new family of algebraic cycles (called Mirković-Vilonen cycles) in the corresponding affine Grassmannian [MV1, MV2]. Furthermore, Breverman and Gaitsgory [BG] showed that a certain set of Mirković-Vilonen cycles has a crystal structure which is isomorphic to the crystal basis of an irreducible highest weight $U_{q}\left(\mathfrak{g}^{\vee}\right)$-module, where $\mathfrak{g}^{\vee}$ is the Langlands dual of $\mathfrak{g}$. By taking the moment map image of Mirković-Vilonen cycles, Anderson [A] defined a family of convex polytopes in $\mathfrak{h}_{\mathbb{R}}$, which are called Mirković-Vilonen $\left(M V\right.$ for short) polytopes. Here $\mathfrak{h}$ is the Cartan subalgebra of $\mathfrak{g}$ and $\mathfrak{h}_{\mathbb{R}}$ the real form of $\mathfrak{h}$.

Key words and phrases. Crystal basis, PBW basis, MV polytopes.

Mathematics Subject Classification (2010): Primary 17B37; Secondary 17B67, 81R10, 81R50.

Reseach of the author is supported by Grant-in-Aid for Scientific Resaerch (C) 20540009, Japan Society for the Promontion Science. 
After these works, Kamnitzer [Kam1], Kam2] gave a combinatorial characterization of MV polytopes by using a notion of Berenstein-Zelevinsky (BZ for short) data. A BZ datum is a certain family of integers (see Section 5 for details), which is introduced by Berenstein, Fomin and Zelevinsky [BFZ]. Moreover he showed that the set of MV polytopes has a crystal structure which is isomorphic to $B(\infty)$. That is, he constructed

(e) a realization of $B(\infty)$ in terms of MV polytopes.

We remark that he also proved that the above crystal structure on the set of MV polytopes coincides with the crystal structure coming from one on the set of Mirković-Vilonen cycles, which is introduced in $[\mathrm{BG}$.

1.3. In the current paper, we focus on the case $\mathfrak{g}=\mathfrak{s} l_{n+1}(\mathbb{C})$. The aim of the paper is to give explicit isomorphisms between the three realizations (c), (d) and (e) of $B(\infty)$. In a process for constructing these isomorphisms, we can also give a quiver theoretical description of MV polytopes (or BZ data) in type $A_{n}$.

As a by-product, we give a new proof of the Anderson-Mirković (AM for short) conjecture. The AM conjecture is a conjecture on the explicit forms of the actions of lowering Kashiwara operators on the set of MV polytopes, which is conjectured by Anderson and Mirković (unpublished) and proved by Kamnitzer [Kam2] (see Theorem 5.5.1).

1.4. This paper is organized as follows. In Section 2, we give a quick review on the theory of crystals. After recalling basic properties of PBW basis of the negative half of quantum enveloping algebras in Section 3, we introduce a crystal structure on PBW basis in Section 4 (see Theorem 4.2.1). We remark that this is just a reformulation of the result of Reineke [Re. In Section 5, after reviewing some of basic facts on MV polytopes following Kamnitzer [Kam1], Kam2], we construct an isomorphism from a parametrizing set of PBW basis (so-called Lusztig data) to the set of MV polytopes in explicit way (see Theorem 5.6.3 which will be proved in Section 7). In other words, this isomorphism tells us an explicit relation between the realization (d) of $B(\infty)$ and (e). In Section 6, we give a quiver theoretical interpretation of a $\mathrm{BZ}$ datum in type $A$ (see Corollary 6.3.3). In this consideration, the work of Berenstein, Fomin and Zelevinsky BFZ plays an important role. In Section 7, as we mentioned above, we give a proof of Theorem 5.6.3. In the first half of this section, we give a short review on a Lagrangian construction of $B(\infty)$, following Kashiwara and the author [KS]. This is just the realization (c). Since the explicit isomorphism between the realization (c) and (d) is already known, the problem can be translated as follows: "prove that the induced map form the realization (c) to (e) is an isomorphism of crystals". In the second half, we prove this problem by using the results of Section 6 and quiver theoretical considerations. Finally, in Section 8, we give a new proof of the AM conjecture in type $A$, as an application of the previous results.

1.5. Very recently, another quiver theoretical interpretation of BZ data was given by Baumann, Kamnitzer and Sadanand ([KamS for type $A$, and $[\mathrm{BK}$ for type $A, D, E$ ). They gave similar results as our article (for example, see Theorem 21 in [BK]). But their approach is different from ours. Indeed, in their interpretation, they use the representation theory of preprojective algebras (in other words, the double quiver of Dynkin type with certain relations). On the other hand, in our construction, we only use the ordinary Dynkin quiver. In addition to that, as we already mentioned above, we focus only on type $A$. By this restriction, we can get an explicit formula for computing each BZ datum in terms of the realization (d) of $B(\infty)$ in type $A$. Consequently, we also have a new proof of the AM conjecture in type $A$. Moreover, our approach can be generalized in affine type $A$ ( $c f$. [NSS1, [NSS2]). In other words, this article is the first step for the above generalization. 
Acknowledgment. The author is grateful to Professor Saburo Kakei, Professor Yoshiyuki Kimura, Professor Satoshi Naito, Professor Daisuke Sagaki and Professor Yoshihiro Takeyama for valuable discussions. The author also would like to thank to Professor Pierre Baumann and Professor Joel Kamnitzer for valuable comments on the earlier draft.

\section{Preliminaries}

2.1. Notations. In this article, we assume $\mathfrak{g}=\mathfrak{s} l_{n+1}(\mathbb{C})$. Let $\mathfrak{h}$ be the Cartan subalgebra of $\mathfrak{g}$. We denote by $\alpha_{i} \in \mathfrak{h}^{*}(i \in I=\{1,2, \cdots, n\})$ the simple roots of $\mathfrak{g}$, and $h_{i} \in \mathfrak{h}(i \in I)$ the simple coroot of $\mathfrak{g}$; note that $\left\langle h_{i}, \alpha_{j}\right\rangle=a_{i j}$ for $i, j \in I$, where $\langle\cdot, \cdot\rangle$ denotes the canonical pairing between $\mathfrak{h}$ and $\mathfrak{h}^{*},\left(a_{i j}\right)_{i, j \in I}$ the Cartan matrix of type $A_{n}$. We denote by $P, Q$ and $\Delta^{+}$the weight lattice, the root lattice and the set of all positive roots, respectively. Let $W=\mathfrak{S}_{n+1}$ be the Weyl group of $\mathfrak{g}$. It is generated by simple reflections $s_{i}=s_{\alpha_{i}}(i \in I)$. Let $e$ and $w_{0}$ be the unit element and the longest element of $W$, respectively.

Let $U_{q}=U_{q}(\mathfrak{g})$ be the quantized universal enveloping algebra of type $A_{n}$ with generators $e_{i}, f_{i}, t_{i}^{ \pm}(i \in I)$. It is an associative algebra over $\mathbb{Q}(q)$. Let $U_{q}^{-}$be the subalgebra of $U_{q}$ generated by $f_{i}(i \in I)$. Define $[l]=\frac{q^{l}-q^{-l}}{q-q^{-1}}$ and $[k] !=\prod_{l=1}^{k}[l]$. For $x \in U_{q}(\mathfrak{g})$, we denote $x^{(k)}=x^{k} /[k] !$.

\subsection{Crystals.}

Definition 2.2.1. (1) Consider the following data:

(i) a set $B$,

(ii) a map wt : $B \rightarrow P$,

(iii) $\operatorname{maps} \varepsilon_{i}: B \rightarrow \mathbb{Z} \sqcup\{-\infty\}, \varphi_{i}: B \rightarrow \mathbb{Z} \sqcup\{-\infty\}(i \in I)$,

(iv) maps $\widetilde{e}_{i}: B \rightarrow B \sqcup\{0\}, \widetilde{f}_{i}: B \rightarrow B \sqcup\{0\}(i \in I)$.

The sixtuple $\left(B ; \mathrm{wt}, \varepsilon_{i}, \varphi_{i}, \widetilde{e}_{i}, \widetilde{f}_{i}\right)$ (denoted by $B$, for short) is called a crystal if it satisfies the following axioms:

(C1) $\varphi_{i}(b)=\varepsilon_{i}(b)+\left\langle h_{i}, \mathrm{wt}(b)\right\rangle$.

(C2) If $b \in B$ and $\widetilde{e}_{i} b \in B$, then $\operatorname{wt}\left(\widetilde{e}_{i} b\right)=\operatorname{wt}(b)+\alpha_{i}, \varepsilon_{i}\left(\widetilde{e}_{i} b\right)=\varepsilon_{i}(b)-1, \varphi_{i}\left(\widetilde{e}_{i} b\right)=$ $\varphi_{i}(b)+1$.

$\left(\mathrm{C} 2{ }^{\prime}\right)$ If $b \in B$ and $\widetilde{f}_{i} b \in B$, then $\operatorname{wt}\left(\widetilde{f}_{i} b\right)=\operatorname{wt}(b)-\alpha_{i}, \varepsilon_{i}\left(\widetilde{f}_{i} b\right)=\varepsilon_{i}(b)+1, \varphi_{i}\left(\widetilde{f}_{i} b\right)=$ $\varphi_{i}(b)-1$.

(C3) For $b, b^{\prime} \in B, b^{\prime}=\widetilde{e}_{i} b$ if and only if $b=\widetilde{f}_{i} b^{\prime}$.

(C4) For $b \in B$, if $\varphi_{i}(b)=-\infty$, then $\widetilde{e}_{i} b=\widetilde{f}_{i} b=0$.

(2) For two crystals $B_{1}$ and $B_{2}$, a morphism $\psi$ from $B_{1}$ to $B_{2}$ is a map $B_{1} \sqcup\{0\} \rightarrow B_{2} \sqcup\{0\}$ that satisfies the following conditions:

(i) $\psi(0)=0$.

(ii) If $b \in B_{1}$ and $\psi(b) \in B_{2}$, then $\operatorname{wt}(\psi(b))=\operatorname{wt}(b), \varepsilon_{i}(\psi(b))=\varepsilon_{i}(b)$ and $\varphi_{i}(\psi(b))=$ $\varphi_{i}(b)$.

(iii) If $b, b^{\prime} \in B_{1}$ satisfy $b^{\prime}=\widetilde{f}_{i}(b)$ and $\varphi(b), \varphi\left(b^{\prime}\right) \in B_{2}$, then $\psi\left(b^{\prime}\right)=\widetilde{f}_{i}(\psi(b))$.

$A$ morphism $\psi: B_{1} \rightarrow B_{2}$ is called an isomorphism, if $\psi$ induces an bijective map $B_{1} \sqcup$ $\{0\} \rightarrow B_{2} \sqcup\{0\}$ and it commutes with all $\widetilde{e}_{i}$ and $\widetilde{f}_{i}$.

2.3. Crystal basis of $U_{q}^{-}$. We shall recall the definition of the crystal basis of $U_{q}^{-}$. Let $e_{i}^{\prime}$ and $e_{i}^{\prime \prime}$ be endomorphisms of $U_{q}^{-}$defined by

$$
\left[e_{i}, x\right]=\frac{t_{i} e_{i}^{\prime \prime}(x)-t_{i}^{-1} e_{i}^{\prime}(x)}{q-q^{-1}} \quad\left(x \in U_{q}^{-}\right) .
$$


It is known that any element $x \in U_{q}^{-}$can be uniquely written as

$$
x=\sum_{k \geq 0} f_{i}^{(k)} x_{k} \quad \text { with } e_{i}^{\prime}\left(x_{k}\right)=0 .
$$

Define modified root operators (so-called Kashiwara operators) $\widetilde{e}_{i}$ and $\widetilde{f}_{i}$ on $U_{q}^{-}$by

$$
\widetilde{e}_{i} x=\sum_{k \geq 1} f_{i}^{(k-1)} x_{k}, \quad \widetilde{f}_{i} x=\sum_{k \geq 0} f_{i}^{(k+1)} x_{k} .
$$

Let $\mathcal{A}$ be the subring of $\mathbb{Q}(q)$ consisting of rational functions without a pole at $q=0$. Set

$$
\begin{gathered}
L(\infty)=\sum_{k \geq 0, i_{1}, \cdots, i_{k} \in I} \mathcal{A} \widetilde{f}_{i_{1}} \cdots \widetilde{f}_{i_{k}} \cdot 1 \subset U_{q}^{-}, \\
B(\infty)=\left\{\widetilde{f}_{i_{1}} \cdots \widetilde{f}_{i_{k}} \cdot 1 \bmod q L(\infty) \mid k \geq 0, i_{1}, \cdots, i_{k} \in I\right\} .
\end{gathered}
$$

Then the following properties hold:

(1) $\widetilde{e}_{i} L(\infty) \subset L(\infty)$ and $\widetilde{f}_{i} L(\infty) \subset L(\infty)$,

(2) $B(\infty)$ is a $\mathbb{Q}$-basis of $L(\infty) / q L(\infty)$,

(3) $\widetilde{e}_{i} B(\infty) \subset B(\infty) \cup\{0\}$ and $\widetilde{f}_{i} B(\infty) \subset B(\infty)$.

We call $(L(\infty), B(\infty))$ the crystal basis of $U_{q}^{-}$.

For $b \in B(\infty)$, we set

$\operatorname{wt}(b)=$ the weight of $b, \quad \varepsilon_{i}(b)=\max \left\{k \geq 0 \mid \widetilde{e}_{i}^{k}(b) \neq 0\right\}, \quad \varphi_{i}(b)=\varepsilon_{i}(b)+\left\langle h_{i}, \operatorname{wt}(b)\right\rangle$.

Then $\left(B(\infty)\right.$, wt, $\left.\varepsilon_{i}, \varphi_{i}, \widetilde{e}_{i}, \widetilde{f}_{i}\right)$ is a crystal in the sense of Definition 2.2.1.

2.4. Orderings on the set of positive roots. Since $\mathfrak{g}=\mathfrak{s} l_{n+1}(\mathbb{C})$, any positive root $\beta \in \Delta^{+}$can be uniquely written as

$$
\beta=\sum_{p=i_{\beta}}^{j_{\beta}-1} \alpha_{p} \quad\left(\text { for some } 1 \leq i_{\beta}<j_{\beta} \leq n+1\right) .
$$

The correspondence $\beta \mapsto\left(i_{\beta}, j_{\beta}\right)$ defines a bijection $\Delta^{+} \stackrel{\sim}{\rightarrow} \Pi$ where

$$
\Pi=\{(i, j) \mid 1 \leq i<j \leq n+1\} .
$$

In the rest of this article, we sometimes identify $\Pi$ with $\Delta^{+}$via the above bijection.

Let $N=n(n+1) / 2$ be the length of $w_{0}$ and fix $w_{0}=s_{i_{1}} s_{i_{2}} \cdots s_{i_{N}}\left(i_{1}, i_{2}, \cdots, i_{N} \in I\right)$ a reduced expression of $w_{0}$. We denote by $\mathbf{i}=\left(i_{1}, i_{2}, \cdots, i_{N}\right)$ the corresponding reduced word. Set $\beta_{k}=s_{i_{1}} s_{i_{2}} \cdots s_{i_{k-1}}\left(\alpha_{i_{k}}\right)(1 \leq k \leq N)$. Then we have $\Pi=\left\{\beta_{1}, \beta_{2}, \cdots, \beta_{N}\right\}$. That is, a reduced word $\mathbf{i}$ defines a bijection $\Upsilon_{\mathbf{i}}:\{1,2, \cdots, N\} \stackrel{\sim}{\rightarrow} \Pi$ and it induces a total ordering $\leq_{\mathrm{i}}$ on $\Pi$;

$$
\left(i_{1}, j_{1}\right) \leq_{\mathbf{i}}\left(i_{2}, j_{2}\right) \stackrel{\text { def }}{\Longleftrightarrow} \Upsilon_{\mathbf{i}}^{-1}\left(i_{1}, j_{1}\right) \leq \Upsilon_{\mathbf{i}}^{-1}\left(i_{2}, j_{2}\right) .
$$

Example 2.4.1. Let $\mathbf{i}_{0}$ be the lexicographically minimal reduced word given by

$$
\mathbf{i}_{0}=(1,2,1,3,2,1, \cdots, n, n-1, \cdots, 1) .
$$

Then we have

$$
\left(i_{1}, j_{1}\right) \leq_{\mathbf{i}_{0}}\left(i_{2}, j_{2}\right) \text { if and only if }\left\{\begin{array}{l}
j_{1}<j_{2} \\
\text { or } \\
j_{1}=j_{2} \text { and } i_{1} \leq i_{2}
\end{array}\right.
$$




\section{PBW BASIS AND LUSZTig DATA ASSOCIATED TO REDUCED WORDS}

3.1. PBW basis of quantized universal enveloping algebras. For $i \in I$, introduce an $\mathbb{Q}(q)$-algebra automorphism $T_{i}$ of $U_{q}$ as :

$$
\begin{gathered}
T_{i}\left(e_{j}\right):= \begin{cases}-f_{i} t_{i} & (i=j), \\
\sum_{k=0}^{-a_{i j}}(-1)^{a_{i j}+k} q^{a_{i j}+k} e_{i}^{(k)} e_{j} e_{i}^{\left(-a_{i j}-k\right)} & (i \neq j),\end{cases} \\
T_{i}\left(f_{j}\right)= \begin{cases}-t_{i}^{-1} e_{i}, & (i=j), \\
\sum_{k=0}^{-a_{i j}}(-1)^{a_{i j}+k} q^{-a_{i j}-k} f_{i}^{\left(-a_{i j}-k\right)} f_{j} f_{i}^{(k)}, & (i \neq j),\end{cases} \\
T_{i}\left(t_{j}\right)=t_{j} t_{i}^{-a_{i j}} .
\end{gathered}
$$

It is known that these operators satisfy the braid relations:

$$
T_{i} T_{j} T_{i}=T_{j} T_{i} T_{j}\left(a_{i j}=-1\right), \quad T_{i} T_{j}=T_{j} T_{i}\left(a_{i j}=0\right) .
$$

For a reduced word $\mathbf{i}=\left(i_{1}, i_{2}, \cdots, i_{N}\right)$ and $\mathbf{c}=\left(c_{1}, \cdots, c_{N}\right) \in \mathbb{Z}_{\geq 0}^{N}$, we define

$$
P_{\mathbf{i}}(\mathbf{c})=f_{i_{1}}^{\left(c_{1}\right)}\left(T_{i_{1}}\left(f_{i_{1}}^{\left(c_{2}\right)}\right)\right) \cdots\left(T_{i_{1}} T_{i_{2}} \cdots T_{i_{N-1}}\left(f_{i_{N}}^{\left(c_{N}\right)}\right)\right)
$$

and

$$
B_{\mathbf{i}}=\left\{P_{\mathbf{i}}(\mathbf{c}) \mid \mathbf{c} \in \mathbb{Z}_{\geq 0}^{N}\right\}, \quad L_{\mathbf{i}}=\sum_{\mathbf{c}} \mathcal{A} P_{\mathbf{i}}(\mathbf{c})
$$

Proposition 3.1.1 ([L1], $[\underline{\mathrm{S}}])$. (1) $B_{\mathbf{i}}$ is a $\mathbb{Q}(q)$-basis of $U_{q}^{-}$.

(2) $L_{\mathbf{i}}=L(\infty)$. Moreover $B_{\mathbf{i}}$ is a free $\mathcal{A}$-basis of $L(\infty)$.

(3) $B_{\mathbf{i}} \equiv B(\infty) \bmod q L(\infty)$.

Definition 3.1.2. For a giving reduced word $\mathbf{i}$, the basis $B_{\mathbf{i}}=\left\{P_{\mathbf{i}}(\mathbf{c}) \mid \mathbf{c} \in \mathbb{Z}_{\geq 0}^{N}\right\}$ is called the $P B W$ basis of $U_{q}^{-}$associated to a reduced word $\mathbf{i}$. There is a bijection $\Xi_{\mathbf{i}}: \mathbb{Z}_{\geq 0}^{N} \stackrel{\sim}{\rightarrow} B(\infty)$ defined by $\mathbf{c} \mapsto P_{\mathbf{i}}(\mathbf{c}) \bmod q L(\infty)$. For $b \in B(\infty)$, we call $\Xi_{\mathbf{i}}^{-1}(b) \in \mathbb{Z}_{\geq 0}^{N}$ the $\mathbf{i}$-Lusztig datum of $b \in B(\infty)$.

3.2. The transition maps. For a reduced word $\mathbf{i}$, consider

$$
\mathcal{B}^{\mathbf{i}}=\left\{\mathbf{a}^{\mathbf{i}}=\left(a_{i, j}^{\mathbf{i}}\right)_{(i, j) \in \Pi} \mid a_{i, j}^{\mathbf{i}} \in \mathbb{Z}_{\geq 0} \text { for any }(i, j) \in \Pi\right\}
$$

the set of all $N$-tuples of non-negative integers indexed by $\Pi$. From now on, we regard $\mathcal{B}^{\mathbf{i}}$ as the set of all $\mathbf{i}$-Lusztig data via the bijection $\mathbb{Z}_{\geq 0}^{N} \stackrel{\sim}{\rightarrow} \mathcal{B}^{\mathbf{i}}$ induced from $\Upsilon_{\mathbf{i}}:\{1, \cdots, N\} \stackrel{\sim}{\rightarrow} \Pi$. For two reduced words $\mathbf{i}$ and $\mathbf{i}^{\prime}$, let us consider the following composition of the bijections:

$$
R_{\mathbf{i}}^{\mathbf{i}^{\prime}}=\Xi_{\mathbf{i}^{\prime}}^{-1} \circ \Xi_{\mathbf{i}}: \mathcal{B}^{\mathbf{i}} \stackrel{\sim}{\rightarrow} B(\infty) \stackrel{\sim}{\rightarrow} \mathcal{B}^{\mathbf{i}^{\prime}}
$$

We call $R_{\mathbf{i}}^{\mathbf{i}^{\prime}}$ the transition map form $\mathbf{i}$ to $\mathbf{i}^{\prime}$. The explicit form of $R_{\mathbf{i}}^{\mathbf{i}^{\prime}}$ is known $([\overline{B F Z}])$, but we omit to give it in this article.

For an arbitrarily reduced word $\mathbf{i}$, the set of all $\mathbf{i}$-Lusztig data $\mathcal{B}^{\mathbf{i}}$ has a crystal structure which is induced form the bijection $\Xi_{\mathbf{i}}: \mathcal{B}^{\mathbf{i}} \stackrel{\sim}{\rightarrow} B(\infty)$. Especially, for the lexicographically minimal reduced word $\mathbf{i}_{0}$, we denote $\mathcal{B}=\mathcal{B}^{\mathbf{i}_{0}}$ and $\mathbf{a}=\mathbf{a}^{\mathbf{i}_{0}} \in \mathcal{B}$. It has a central role in this article. In the next section we will give the crystal structure on $\mathcal{B}=\mathcal{B}^{\mathbf{i}_{0}}$ in explicit way. 
3.3. *-structure. Define a $\mathbb{Q}(q)$-algebra anti-involution $*$ of $U_{q}$ by

$$
e_{i}^{*}=e_{i}, \quad f_{i}^{*}=f_{i}, \quad\left(t_{i}^{ \pm}\right)^{*}=t_{i}^{\mp} \quad(i \in I) .
$$

By the construction, it is easy to see $L(\infty)^{*}=L(\infty)$. Therefore $*$ induces a $\mathbb{Q}$-linear automorphism of $L(\infty) / q L(\infty)$. Moreover the following theorem is known.

Theorem 3.3.1 ([K3]). The anti-involution $*$ induces an involutive bijection on $B(\infty)$.

By the above theorem, we can define the operators $\widetilde{e}_{i}^{*}, \widetilde{f}_{i}^{*}$ on $B(\infty)$ by

$$
\widetilde{e}_{i}^{*}=* \widetilde{e}_{i} *, \quad \widetilde{f}_{i}^{*}=* \widetilde{f}_{i} * .
$$

By the definition, it is obvious that $b_{\infty}^{*}=b_{\infty}$. Here $b_{\infty}$ is the highest weight element of $B(\infty)$. Therefore we have

$$
\varepsilon_{i}\left(b^{*}\right)=\max \left\{k \geq 0 \mid\left(\widetilde{e}_{i}^{*}\right)^{k}(b) \neq 0\right\}
$$

for $b \in B(\infty)$. From now on, we denote $\varepsilon_{i}^{*}(b)=\varepsilon_{i}\left(b^{*}\right)$.

Define a $\mathbb{Q}(q)$-algebra automorphism $T_{i}^{*}(i \in I)$ by

$$
T_{i}^{*}=* \circ T_{i} \circ *
$$

Then we have

$$
\begin{aligned}
P_{\mathbf{i}}^{*}(\mathbf{c}) & =\left(f_{i_{1}}^{\left(c_{1}\right)}\left(T_{i_{1}}\left(f_{i_{1}}^{\left(c_{2}\right)}\right)\right) \cdots\left(T_{i_{1}} T_{i_{2}} \cdots T_{i_{N-1}}\left(f_{i_{N}}^{\left(c_{N}\right)}\right)\right)\right)^{*} \\
& =\left(T_{i_{1}}^{*} T_{i_{2}}^{*} \cdots T_{i_{N-1}}^{*}\left(f_{i_{N}}^{\left(c_{N}\right)}\right)\right) \cdots\left(T_{i_{1}}^{*}\left(f_{i_{1}}^{\left(c_{2}\right)}\right)\right) f_{i_{1}} .
\end{aligned}
$$

Set

$$
B_{\mathbf{i}}^{*}=\left\{P_{\mathbf{i}}^{*}(\mathbf{c}) \mid \mathbf{c} \in \mathbb{Z}_{\geq 0}^{N}\right\} .
$$

It also gives a $\mathbb{Q}(q)$-basis of $U_{q}^{-}$. We call it the $*-P B W$ basis of $U_{q}^{-}$associated to a reduced word $\mathbf{i}$.

By Theorem 3.3.1, we have the following corollary.

Corollary 3.3.2.

$$
\left\{P_{\mathbf{i}}^{*}(\mathbf{c}) \bmod q L(\infty) \mid \mathbf{c} \in \mathbb{Z}_{\geq 0}^{N}\right\}=B(\infty) .
$$

\section{Crystal structure on $\mathbf{i}_{0}$-Lusztig data}

4.1. Definition of crystal structures. We shall define two crystal structures on the set of all $\mathbf{i}_{0}$-Lusztig datum $\mathcal{B}$. For $\mathbf{a} \in \mathcal{B}$, define the weight wt(a) of $\mathbf{a}$ by

$$
\mathrm{wt}(\mathbf{a})=-\sum_{i \in I} m_{i} \alpha_{i}, \quad \text { where } \quad m_{i}=\sum_{k=1}^{i} \sum_{l=i+1}^{n+1} a_{k, l} \quad(i \in I) .
$$

For $i \in I$, set

$$
\begin{gathered}
A_{k}^{(i)}(\mathbf{a})=\sum_{s=1}^{k}\left(a_{s, i+1}-a_{s-1, i}\right) \quad(1 \leq k \leq i), \\
A_{l}^{*(i)}(\mathbf{a})=\sum_{t=l+1}^{n+1}\left(a_{i, t}-a_{i+1, t+1}\right) \quad(i \leq l \leq n) .
\end{gathered}
$$

Here we set $a_{0, i}=0$ and $a_{i+1, n+2}=0$. Define

$$
\varepsilon_{i}(\mathbf{a})=\max \left\{A_{1}^{(i)}(\mathbf{a}), \cdots, A_{i}^{(i)}(\mathbf{a})\right\}, \quad \varphi_{i}(\mathbf{a})=\varepsilon_{i}(\mathbf{a})+\left\langle h_{i}, \operatorname{wt}(\mathbf{a})\right\rangle,
$$




$$
\varepsilon_{i}^{*}(\mathbf{a})=\max \left\{A_{i}^{*(i)}(\mathbf{a}), \cdots, A_{n}^{*(i)}(\mathbf{a})\right\}, \quad \varphi_{i}^{*}(\mathbf{a})=\varepsilon_{i}^{*}(\mathbf{a})+\left\langle h_{i}, \operatorname{wt}(\mathbf{a})\right\rangle .
$$

Let

$$
\begin{aligned}
& k_{e}=\min \left\{1 \leq k \leq i \mid \varepsilon_{i}(\mathbf{a})=A_{k}^{(i)}(\mathbf{a})\right\}, \quad k_{f}=\max \left\{1 \leq k \leq i \mid \varepsilon_{i}(\mathbf{a})=A_{k}^{(i)}(\mathbf{a})\right\}, \\
& l_{e}=\max \left\{i \leq l \leq n \mid \varepsilon_{i}^{*}(\mathbf{a})=A_{l}^{*(i)}(\mathbf{a})\right\}, \quad l_{f}=\min \left\{i \leq l \leq n \mid \varepsilon_{i}^{*}(\mathbf{a})=A_{l}^{*(i)}(\mathbf{a})\right\} .
\end{aligned}
$$

For a given $\mathbf{a} \in \mathcal{B}$, we introduce four $N$-tuples of integers $\mathbf{a}^{(p)}=\left(a_{k, l}^{(p)}\right)(p=1,2,3,4)$ by

$$
\begin{aligned}
& a_{k, l}^{(1)}= \begin{cases}a_{k_{e}, i}+1 & \left(k=k_{e}, l=i\right), \\
a_{k_{e}, i+1}-1 & \left(k=k_{e}, l=i+1\right), \\
a_{k, l} & (\text { otherwise })\end{cases} \\
& a_{k, l}^{(2)}= \begin{cases}a_{k_{f}, i}-1 & \left(k=k_{f}, l=i\right), \\
a_{k_{f}, i+1}+1 & \left(k=k_{f}, l=i+1\right), \\
a_{k, l} & (\text { otherwise }),\end{cases} \\
& a_{k, l}^{(3)}= \begin{cases}a_{i, l_{e}+1}-1 & \left(k=i, l=l_{e}+1\right), \\
a_{i+1, l_{e}+1}+1 & \left(k=i+1, l=l_{e}+1\right), \\
a_{k, l} & (\text { otherwise }) .\end{cases} \\
& a_{k, l}^{(4)}= \begin{cases}a_{i, l_{f}+1}+1 & \left(k=i, l=l_{f}+1\right), \\
a_{i+1, l_{f}+1}-1 & \left(k=i+1, l=l_{f}+1\right), \\
a_{k, l} & (\text { otherwise }) .\end{cases}
\end{aligned}
$$

Lemma 4.1.1. (1) For any $\mathbf{a} \in \mathcal{B}$ with $\varepsilon_{i}(\mathbf{a})>0$, $\mathbf{a}^{(1)}$ is an $N$-tuple of non-negative integers. In other words, $\mathbf{a}^{(1)}$ is an element of $\mathcal{B}$.

(2) For any $\mathbf{a} \in \mathcal{B}$ with $\varepsilon_{i}^{*}(\mathbf{a})>0, \mathbf{a}^{(3)}$ is an element of $\mathcal{B}$.

(3) For any $\mathbf{a} \in \mathcal{B}$, both $\mathbf{a}^{(2)}$ and $\mathbf{a}^{(4)}$ are elements of $\mathcal{B}$.

Proof. We only give a proof of (1). It is enough to show that $a_{k_{e}, i+1}>0$. If $k_{e}=1$, we have $a_{k_{e}, i+1}=A_{1}^{(i)}(\mathbf{a})=\varepsilon_{i}(\mathbf{a})>0$. Assume $k_{e}>1$. Then, by the definition of $k_{e}$, we have $A_{k_{e}-1}^{(i)}(\mathbf{a})<A_{k_{e}}^{(i)}(\mathbf{a})$. Therefore $A_{k_{e}}^{(i)}(\mathbf{a})-A_{k_{e}-1}^{(i)}(\mathbf{a})=a_{k_{e}, i+1}-a_{k_{e}-1, i}>0$. Since $a_{k_{e}-1, i} \geq 0$, we have $a_{k_{e}, i+1}>0$.

Now we define Kashiwara operators on $\mathcal{B}$ as:

$$
\begin{aligned}
& \widetilde{e}_{i} \mathbf{a}= \begin{cases}0 & \left(\text { if } \varepsilon_{i}(\mathbf{a})=0\right), \\
\mathbf{a}^{(1)} & \left(\text { if } \varepsilon_{i}(\mathbf{a})>0\right),\end{cases} \\
& \widetilde{e}_{i}^{*} \mathbf{a}= \begin{cases}0 & \left(\text { if } \varepsilon_{i}^{*}(\mathbf{a})=0\right), \\
\mathbf{a}^{(3)} & \left(\text { if } \varepsilon_{i}^{*}(\mathbf{a})>0\right),\end{cases}
\end{aligned}
$$

Proposition 4.1.2. (1) (B, wt, $\left.\varepsilon_{i}, \varphi_{i}, \widetilde{e}_{i}, \widetilde{f}_{i}\right)$ is a crystal in the sense of Definition 2.2.1. (2) $\left(\mathcal{B}, \mathrm{wt}, \varepsilon_{i}^{*}, \varphi_{i}^{*}, \widetilde{e}_{i}^{*}, \widetilde{f}_{i}^{*}\right)$ is a crystal in the sense of Definition 2.2.1.

From the definition, one can easily check the axiom $(\mathrm{C} 1) \sim(\mathrm{C} 4)$. So we omit to give a detail.

4.2. Crystal structure on PBW basis associated to $\mathbf{i}_{0}$. As we mentioned in the previous subsection, we regard $\mathcal{B}$ as the set of $\mathbf{i}_{0}$-Lusztig datum and denote by $\left\{P_{\mathbf{i}_{0}}(\mathbf{a}) \mid \mathbf{a} \in\right.$ $\mathcal{B}\}$ the corresponding PBW basis. 
Theorem 4.2.1. (1) We have

$$
\widetilde{e}_{i} P_{\mathbf{i}_{0}}(\mathbf{a}) \equiv P_{\mathbf{i}_{0}}\left(\widetilde{e}_{i} \mathbf{a}\right) \bmod q L(\infty) \quad \text { and } \quad \widetilde{f}_{i} P_{\mathbf{i}_{0}}(\mathbf{a}) \equiv P_{\mathbf{i}_{0}}\left(\widetilde{f}_{i} \mathbf{a}\right) \bmod q L(\infty)
$$

(2) We have

$$
\widetilde{e}_{i} P_{\mathbf{i}_{0}}^{*}(\mathbf{a}) \equiv P_{\mathbf{i}_{0}}^{*}\left(\widetilde{e}_{i}^{*} \mathbf{a}\right) \bmod q L(\infty) \quad \text { and } \quad \widetilde{f}_{i} P_{\mathbf{i}_{0}}^{*}(\mathbf{a}) \equiv P_{\mathbf{i}_{0}}^{*}\left(\widetilde{f}_{i}^{*} \mathbf{a}\right) \bmod q L(\infty) .
$$

Remark . The formulas (2) is proved by Reineke [Re] (see also [Sav], EK]). Note that in $\left[\mathrm{Re}\right.$, he denotes our $\widetilde{e}_{i}^{*}$ and $\widetilde{f}_{i}^{*}$ on $\mathcal{B}$, by $\widetilde{e}_{i}$ and $\widetilde{f}_{i}$, respectively. The formulas (1) is proved by the similar method.

By the definition, we immediately have the next corollary.

Corollary 4.2.2. (1) We have

$$
\widetilde{e}_{i}^{*} P_{\mathbf{i}_{0}}(\mathbf{a}) \equiv P_{\mathbf{i}_{0}}\left(\widetilde{e}_{i}^{*} \mathbf{a}\right) \bmod q L(\infty) \quad \text { and } \quad \widetilde{f}_{i}^{*} P_{\mathbf{i}_{0}}(\mathbf{a}) \equiv P_{\mathbf{i}_{0}}\left(\widetilde{f}_{i}^{*} \mathbf{a}\right) \bmod q L(\infty) .
$$

(2) As a by-product, we have that $\mathcal{B}$ is isomorphic to $B(\infty)$ as a crystal with $*$-structure.

\section{Mirković-Vilonen polytopes in type $A_{n}$}

5.1. Mirković-Vilonen polytopes and Berenstein-Zelevinsky data. Let $\Lambda_{i}(i \in I)$ be a fundamental weight for $\mathfrak{g}$. Set

$$
\Gamma_{n}=\bigcup_{i \in I} W \Lambda_{i}
$$

and an element $\gamma \in \Gamma_{n}$ is called a chamber weight. Let $\mathbf{M}=\left(M_{\gamma}\right)_{\gamma \in \Gamma_{n}}$ be a collection of integers indexed by $\Gamma_{n}$. For each $\gamma \in \Gamma_{n}$, we call $M_{\gamma}$ the the $\gamma$-component of $\mathbf{M}$, and denote it by $(\mathbf{M})_{\gamma}$.

For a given $\mathbf{M}=\left(M_{\gamma}\right)_{\gamma \in \Gamma_{n}}$, consider the following polytope in $\mathfrak{h}_{\mathbb{R}}$ :

$$
P(\mathbf{M})=\left\{h \in \mathfrak{h}_{\mathbb{R}} \mid\langle h, \gamma\rangle \geq M_{\gamma}\left(\forall \gamma \in \Gamma_{n}\right)\right\} .
$$

Definition 5.1.1. (1) A polytope $P(\mathbf{M})$ is called a pseudo-Weyl polytope if it satisfies the following condition:

(BZ-1) (edge inequalities) for all $w \in W$ and $i \in I$,

$$
M_{w \Lambda_{i}}+M_{w s_{i} \Lambda_{i}}+\sum_{j \in I \backslash\{i\}} a_{j i} M_{w \Lambda_{j}} \leq 0
$$

(2) A pseudo-Weyl polytope $P(\mathbf{M})$ is called a Mirković-Vilonen ( $M V$ for short) polytope if it satisfies the following condition:

(BZ-2) (3-term relations) for every $w \in W$ and $i, j \in I$ with $a_{i j}=a_{j i}=-1$ and $w s_{i}>w$, $w s_{j}>w$,

$$
M_{w s_{i} \Lambda_{i}}+M_{w s_{j} \Lambda_{j}}=\min \left\{M_{w \Lambda_{i}}+M_{w s_{i} s_{j} \Lambda_{j}}, M_{w \Lambda_{j}}+M_{w s_{j} s_{i} \Lambda_{i}}\right\} .
$$

Here $\left(a_{i j}\right)_{i, j \in I}$ is the Cartan matrix of type $A_{n}$ and $>$ is the strong Bruhat ordering of $W$. If $P(\mathbf{M})$ is a $M V$ polytope, the corresponding collection of integers $\mathbf{M}=\left(M_{\gamma}\right)_{\gamma \in \Gamma_{n}}$ is called Berenstein-Zelevinsky (BZ for short) datum of type $A_{n}$.

Remark . For a collection of integers $\mathbf{M}=\left(M_{\gamma}\right)_{\gamma \in \Gamma_{n}}$ which satisfies the condition (BZ-1), set

$$
\mu_{w}=\sum_{i \in I} M_{w \Lambda_{i}} w h_{i} \in \mathfrak{h}_{\mathbb{R}} \quad(w \in W)
$$

and consider a collection of vectors

$$
\mu_{\bullet}=\left(\mu_{w}\right)_{w \in W} \subset \mathfrak{h}_{\mathbb{R}} .
$$


Then Kamnitzer [Kam2] showed the corresponding pseudo-Weyl polytope $P(\mathbf{M})$ is the convex hull of $\mu_{\bullet}$. That is, there is a one to one correspondence between the set of pseudoWeyl polytopes and the set of collections of integers which satisfies the condition (BZ-1).

Definition 5.1.2. A BZ datum $\mathbf{M}^{w_{0}}=\left(M_{\gamma}^{w_{0}}\right)_{\gamma \in \Gamma_{n}}$ is called a $w_{0}$-BZ datum of type $A_{n}$ if it satisfies

(BZ-0) (wo-normalization condition) for all $i \in I$,

$$
M_{w_{0} \Lambda_{i}}^{w_{0}}=0
$$

We denote by $\mathcal{B Z}^{w_{0}}$ the set of all $w_{0}-B Z$ data.

A set of integers $K \subset[1, n+1]$ will be called a Maya diagram of rank $n$. We denote by $\mathcal{M}_{n}$ the set of all Maya diagram of rank $n$. Set $\mathcal{M}_{n}^{\times}=\mathcal{M}_{n} \backslash\{\phi,[1, n+1]\}$. From now on, we identify the set of chamber weights $\Gamma_{n}$ with $\mathcal{M}_{n}^{\times}$by the following way: recall that there is a natural action of $W \cong \mathfrak{S}_{n+1}$ on the set $\{1,2, \cdots, n+1\}$. Consider the map $\Gamma_{n} \rightarrow \mathcal{M}_{n}^{\times}$defined by $\gamma=w \Lambda_{i} \mapsto w \cdot[1, i]$. Since this map is bijective, we can identify $\Gamma_{n}$ with $\mathcal{M}_{n}^{\times}$. By the above identification, $\Lambda_{i}$ and $w_{0} \Lambda_{i}$ are regarded as

$$
\Lambda_{i} \leftrightarrow[1, i], \quad w_{0} \Lambda_{i} \leftrightarrow[n-i+2, n+1] \quad(i \in I) .
$$

Under the above identification, the definition of $w_{0}$-BZ datum can be rewritten as follows:

Lemma 5.1.3. A collection $\mathbf{M}^{w_{0}}=\left(M_{K}^{w_{0}}\right)_{K \in \mathcal{M}_{n}^{\times}}$of integers is a $w_{0}-B Z$ datum of type $A_{n}$ if and only if it satisfies the following conditions:

(BZ-0)' for all $i \in I$,

$$
M_{[n-i+2, n+1]}^{w_{0}}=0
$$

(BZ-1)' for every two indices $i \neq j$ in $[1, n+1]$ and every $K \in \mathcal{M}_{n}$ with $K \cap\{i, j\}=\phi$,

$$
M_{K i}^{w_{0}}+M_{K j}^{w_{0}} \leq M_{K i j}^{w_{0}}+M_{K}^{w_{0}}
$$

(BZ-2)' for every three indices $i<j<k$ in $[1, n+1]$ and every $K \in \mathcal{M}_{n}$ with $K \cap\{i, j, k\}=$ $\phi$,

$$
M_{K i k}^{w_{0}}+M_{M j}^{w_{0}}=\min \left\{M_{K i j}^{w_{0}}+M_{K k}^{w_{0}}, M_{K j k}^{w_{0}}+M_{K i}^{w_{0}}\right\} .
$$

Here we denote $M_{K i}^{w_{0}}=M_{K \cup\{i\}}^{w_{0}}$, etc., and set $M_{\phi}^{w_{0}}=M_{[1, n+1]}^{w_{0}}=0$.

Remark . The conditions (BZ-2)' are just the conditions which are called the 3-term relations in [BFZ].

\section{2. e-BZ datum.}

Definition 5.2.1. A collection $\mathbf{M}^{e}=\left(M_{K}^{e}\right)_{K \in \mathcal{M}_{n}^{\times}}$of integers is called a e-normalized Berenstein-Zelevinsky (e-BZ for short) datum of type $A_{n}$ if it satisfies the above (BZ-1)', (BZ-2)' and

(BZ-0)" (e-normalization condition) for all $i \in I$,

$$
M_{[1, i]}^{e}=0 .
$$

We denote by $\mathcal{B Z}^{e}$ the set of all e-BZ data.

For $K \in \mathcal{M}_{n}^{\times}$, let $K^{c}=[1, n+1] \backslash K$ be the complement of $K$ in $[1, n+1]$. For $\mathbf{M}^{w_{0}}=\left(M_{K}^{w_{0}}\right)_{K \in \mathcal{M}_{n}^{\times}} \in \mathcal{B Z}^{w_{0}}$, we define a new collection of integers $\mathbf{M}^{w_{0} *}=\left(M_{K}^{w_{0} *}\right)_{K \in \mathcal{M}_{n}^{\times}}$ by

$$
M_{K}^{w_{0 *}}=M_{K^{c}}^{w_{0}}\left(K \in \mathcal{M}_{n}^{\times}\right)
$$


Lemma 5.2.2. The map $*: \mathbf{M}^{w_{0}} \mapsto \mathbf{M}^{w_{0} *}$ defines a bijection form $\mathcal{B Z}^{w_{0}}$ to $\mathcal{B Z}^{e}$. The inverse $\mathcal{B Z}^{e} \rightarrow \mathcal{B Z}^{w_{0}}$ of the map $*$ is given by

$$
\mathbf{M}^{e}=\left(M_{K}^{e}\right) \mapsto \mathbf{M}^{e *}=\left(M_{K}^{e *}\right), \quad \text { where } M_{K}^{e *}=M_{K^{c}}^{e}\left(K \in \mathcal{M}_{n}^{\times}\right) .
$$

Proof. Let $\mathbf{M}^{w_{0}}=\left(M_{K}^{w_{0}}\right) \in \mathcal{B Z}_{w_{0}}$. Then it is clear that the collection of integers $\mathbf{M}^{w_{0} *}=$ $\left(M_{K}^{w_{0} *}\right)$ satisfies (BZ-0)". Let us prove that $\mathbf{M}^{w_{0} *}$ satisfies (BZ-1)'. Let $i \neq j$ be two indices in $[1, n+1]$ and $K \in \mathcal{M}_{n}$ with $K \cap\{i, j\}=\phi$. For such $i, j$ and $K$, we set $L=K^{c} \backslash\{i, j\}$. Then we have $L \in \mathcal{M}_{n}$ and $L \cap\{i, j\}=\phi$. Since $\mathbf{M}^{w_{0}}$ satisfies (BZ-1)', we have

Because

$$
M_{L i}^{w_{0}}+M_{L j}^{w_{0}} \leq M_{L i j}^{w_{0}}+M_{L}^{w_{0}}
$$

$$
K^{c}=L i j, \quad(K i)^{c}=L j, \quad(K j)^{c}=L i, \quad(K i j)^{c}=L,
$$

we have

$$
M_{K}^{w_{0 *}}=M_{L i j}^{w_{0}}, \quad M_{K i}^{w_{0} *}=M_{L j}^{w_{0}}, \quad M_{K j}^{w_{0 *}}=M_{L i}^{w_{0}}, \quad M_{K i j}^{w_{0 *}}=M_{L}^{w_{0}} .
$$

Therefore we have

$$
M_{K j}^{w_{0} *}+M_{K i}^{w_{0} *} \leq M_{K}^{w_{0 *}}+M_{K i j}^{w_{0} *}
$$

This is nothing but (BZ-1)' for $\mathbf{M}^{w_{0} *}$.

By the similar argument we can check (BZ-2)' for $\mathbf{M}^{w_{0} *}$. Thus, $\mathbf{M}^{w_{0} *}$ is an $e-\mathrm{BZ}$ datum. The other statements are clear by the construction.

5.3. Crystal structure on $w_{0}$-BZ data. We denote $\mathcal{M V}=\left\{P\left(\mathbf{M}^{w_{0}}\right) \mid \mathbf{M}^{w_{0}} \in \mathcal{B Z}^{w_{0}}\right\}$. In Kam2, Kamnitzer defines a crystal structure on $\mathcal{M V}$ and shows it is isomorphic to $B(\infty)$ as a crystal. Since the map $\mathcal{B Z}^{w_{0}} \rightarrow \mathcal{M V}$ defined by $\mathbf{M}^{w_{0}} \mapsto P\left(\mathbf{M}^{w_{0}}\right)$ is bijective, we can define a crystal structure on $\mathcal{B Z}^{w_{0}}$ in such a way that the above bijection gives an isomorphism of crystals. In the following, we recall the description of this crystal structure on $\mathcal{B Z}^{w_{0}}$ form [Kam2].

Remark . In [Kam2], he uses the set of chamber weights $\Gamma_{n}$ as the index set of $\mathcal{B Z}^{w_{0}}$. But, for later use, we will reformulate the above crystal structure on $\mathcal{B Z}^{w_{0}}$ by using the set of Maya diagrams $\mathcal{M}_{n}^{\times}$instead of $\Gamma_{n}$.

Let $\mathbf{M}^{w_{0}}=\left(M_{K}^{w_{0}}\right) \in \mathcal{B} \mathcal{Z}^{w_{0}}$. Define the weight $\operatorname{wt}\left(\mathbf{M}^{w_{0}}\right)$ of $\mathbf{M}^{w_{0}}$ by

$$
\operatorname{wt}\left(\mathbf{M}^{w_{0}}\right)=\sum_{i \in I} M_{[1, i]}^{w_{0}} \alpha_{i} .
$$

For $i \in I$, we set

$$
\begin{aligned}
& \varepsilon_{i}\left(\mathbf{M}^{w_{0}}\right)=-\left(M_{[1, i]}^{w_{0}}+M_{[1, i+1] \backslash\{i\}}^{w_{0}}-M_{[1, i+1]}^{w_{0}}-M_{[1, i] \backslash\{i\}}^{w_{0}}\right), \\
& \varphi_{i}\left(\mathbf{M}^{w_{0}}\right)=\varepsilon_{i}\left(\mathbf{M}^{w_{0}}\right)+\left\langle h_{i}, \operatorname{wt}\left(\mathbf{M}^{w_{0}}\right)\right\rangle .
\end{aligned}
$$

We remark that $\varepsilon_{i}\left(\mathbf{M}^{w_{0}}\right)$ is a non-negative integer in view of (BZ-1)'.

Let us define the action of Kashiwara operators $\widetilde{e}_{i}$ and $\widetilde{f}_{i}(i \in I)$. We recall the following fact due to Kamnitzer:

Proposition 5.3.1 ([Kam2] $)$. Let $\mathbf{M}^{w_{0}}=\left(M_{K}^{w_{0}}\right) \in \mathcal{B Z}^{w_{0}}$ be a $w_{0}$-BZ datum.

(1) If $\varepsilon_{i}\left(\mathbf{M}^{w_{0}}\right)>0$, there exists a unique $w_{0}-B Z$ datum which is denoted by $\widetilde{e}_{i} \mathbf{M}^{w_{0}}$ such that

(i) $\left(\widetilde{e}_{i} \mathbf{M}^{w_{0}}\right)_{[1, i]}=M_{[1, i]}^{w_{0}}+1$,

(ii) $\left(\widetilde{e}_{i} \mathbf{M}^{w_{0}}\right)_{K}=M_{K}^{w_{0}}$ for all $K \in \mathcal{M}_{n}^{\times} \backslash \mathcal{M}_{n}^{\times}(i)$.

Here $\mathcal{M}_{n}^{\times}(i)=\left\{K \in \mathcal{M}_{n}^{\times} \mid i \in K\right.$ and $\left.i+1 \notin K\right\} \subset \mathcal{M}_{n}^{\times}$.

(2) There exists a unique a unique $w_{0}-B Z$ datum which is denoted by $\widetilde{f}_{i} \mathbf{M}^{w_{0}}$ such that 
(iii) $\left(\widetilde{f}_{i} \mathbf{M}^{w_{0}}\right)_{[1, i]}=M_{[1, i]}^{w_{0}}-1$,

(iv) $\left(\widetilde{f_{i}} \mathbf{M}^{w_{0}}\right)_{K}=M_{K}^{w_{0}}$ for all $K \in \mathcal{M}_{n}^{\times} \backslash \mathcal{M}_{n}^{\times}(i)$.

If $\varepsilon_{i}\left(\mathbf{M}^{w_{0}}\right)=0$, we set $\widetilde{e}_{i} \mathbf{M}^{w_{0}}=0$.

Theorem 5.3.2 ( $(\underline{\operatorname{Kam} 2}) \cdot\left(\mathcal{B Z}^{w_{0}}, \mathrm{wt}, \varepsilon_{i}, \varphi_{i}, \widetilde{e}_{i}, \widetilde{f}_{i}\right)$ is a crystal in the sense of Definition 2.2.1, which is isomorphic to $B(\infty)$.

5.4. *-crystal structure on $e$-BZ data. By using Theorem 5.3.2, we can define a crystal structure on the set of $e$-BZ data $\mathcal{B Z}^{e}$ as follows. Recall the bijection $*: \mathcal{B Z}^{w_{0}} \stackrel{\sim}{\rightarrow} \mathcal{B Z}^{e}$ and its inverse which is also denoted by $*$. For $\mathbf{M}^{e} \in \mathcal{B} \mathcal{Z}^{e}$, we set

$$
\mathrm{wt}\left(\mathbf{M}^{e}\right)=\operatorname{wt}\left(\mathbf{M}^{e *}\right), \quad \varepsilon_{i}^{*}\left(\mathbf{M}^{e}\right)=\varepsilon_{i}\left(\mathbf{M}^{e *}\right), \quad \varphi_{i}^{*}\left(\mathbf{M}^{e}\right)=\varphi_{i}\left(\mathbf{M}^{e *}\right) .
$$

Here we remark that $\mathbf{M}^{e *}$ is a $w_{0}$-BZ datum and the right hand sides are already defined. The Kashiwara operators $\widetilde{e}_{i}^{*}, \widetilde{f}_{i}^{*}(i \in I)$ on $\mathcal{B Z}^{e}$ are defined by

$$
\widetilde{e}_{i}^{*}=* \circ \widetilde{e}_{i} \circ *, \quad \widetilde{f}_{i}^{*}=* \circ \widetilde{f}_{i} \circ * .
$$

The following corollary is an easy consequence of Proposition 5.3.1 and Theorem 5.3.2.

Corollary 5.4.1. (1) Let $\mathbf{M}^{e}=\left(M_{K}^{e}\right) \in \mathcal{B} \mathcal{Z}^{e}$ be an e-BZ datum. If $\varepsilon_{i}^{*}\left(\mathbf{M}^{e}\right)>0$, there exists a unique e-BZ datum which is denoted by $\widetilde{e}_{i}^{*} \mathbf{M}^{e}$ such that

(i) $\left(\widetilde{e}_{i}^{*} \mathbf{M}^{e}\right)_{[1, i]^{c}}=M_{[1, i]^{c}}^{e}+1$,

(ii) $\left(\widetilde{e}_{i}^{*} \mathbf{M}^{e}\right)_{K}=M_{K}^{e}$ for all $K \in \mathcal{M}_{n}^{\times} \backslash \mathcal{M}_{n}^{\times}(i)^{*}$.

Here $\mathcal{M}_{n}^{\times}(i)^{*}=\left\{K \in \mathcal{M}_{n}^{\times} \mid i \notin K\right.$ and $\left.i+1 \in K\right\} \subset \mathcal{M}_{n}^{\times}$.

(2) There exists a unique a unique e-BZ datum which is denoted by $\tilde{f}_{i}^{*} \mathbf{M}^{e}$ such that

(iii) $\left(\tilde{f}_{i}^{*} \mathbf{M}^{e}\right)_{[1, i]^{c}}=M_{[1, i]^{c}}^{e}-1$,

(iv) $\left(\tilde{f}_{i}^{*} \mathbf{M}^{e}\right)_{K}=M_{K}^{e}$ for all $K \in \mathcal{M}_{n}^{\times} \backslash \mathcal{M}_{n}^{\times}(i)^{*}$.

(3) $\left(\mathcal{B Z}^{e}, \mathrm{wt}, \varepsilon_{i}^{*}, \varphi_{i}^{*}, \widetilde{e}_{i}^{*}, \widetilde{f}_{i}^{*}\right)$ is a crystal in the sense of Definition 2.2.1, which is isomorphic to $B(\infty)$.

5.5. Anderson-Mirković conjecture. Let $\mathbf{M}^{w_{0}}=\left(M_{K}^{w_{0}}\right) \in \mathcal{B Z}^{w_{0}}$ be be a $w_{0}$-BZ datum. In Kam2, Kamnitzer gives the the explicit form of $\widetilde{f}_{i} \mathbf{M}^{w_{0}}$. We shall recall his result under the identification $\Gamma_{n} \cong \mathcal{M}_{n}^{\times}$.

Theorem 5.5.1 ([Kam2]). For each $i \in I$, we have

$$
\left(\widetilde{f}_{i} \mathbf{M}^{w_{0}}\right)_{K}= \begin{cases}\min \left\{M_{K}^{w_{0}}, M_{s_{i} K}^{w_{0}}+c_{i}\left(\mathbf{M}^{w_{0}}\right)\right\} & \left(K \in \mathcal{M}_{n}^{\times}(i)\right), \\ M_{K}^{w_{0}} & \text { (otherwise) } .\end{cases}
$$

Here $c_{i}\left(\mathbf{M}^{w_{0}}\right)=M_{[1, i]}^{w_{0}}-M_{[1, i+1] \backslash\{i\}}^{w_{0}}-1$.

Remark . (1) If $K=[1, i]$, then we have $\left(\widetilde{f}_{i} \mathbf{M}^{w_{0}}\right)_{[1, i]}=M_{[1, i]}^{w_{0}}-1$. Indeed, $[1, i]$ is an element of $\mathcal{M}_{n}^{\times}(i)$. Since $s_{i}[1, i]=[1, i+1] \backslash\{i\}$, we have

$$
\begin{aligned}
\left(\tilde{f}_{i} \mathbf{M}^{w_{0}}\right)_{[1, i]} & =\min \left\{M_{[1, i]}^{w_{0}}, M_{[1, i+1] \backslash\{i\}}^{w_{0}}+M_{[1, i]}^{w_{0}}-M_{[1, i+1] \backslash\{i\}}^{w_{0}}-1\right\} \\
& =\min \left\{M_{[1, i]}^{w_{0}}, M_{[1, i]}^{w_{0}}-1\right\} \\
& =M_{[1, i]}^{w_{0}}-1 .
\end{aligned}
$$

(2) As we already mentioned in the introduction, the above formula is conjectured by Anderson and Mirković (unpublished) (See [Kam2]). So it is called the Anderson-Mirković (AM for short) conjecture. 
By using the above formula, we can also calculate the explicit form of the $\widetilde{f}_{i}^{*}$-action on an $e$-BZ datum.

Corollary 5.5.2. For $\mathbf{M}^{e}=\left(M_{K}^{e}\right) \in \mathcal{B Z}^{e}$ we have

$$
\left(\tilde{f}_{i}^{*} \mathbf{M}^{e}\right)_{K}= \begin{cases}\min \left\{M_{K}^{e}, M_{s_{i}}^{e}+c_{i}^{*}\left(\mathbf{M}^{e}\right)\right\} & \left(K \in \mathcal{M}_{n}^{\times}(i)^{*}\right) \\ M_{K}^{e} & \text { (otherwise) }\end{cases}
$$

Here $c_{i}^{*}\left(\mathbf{M}^{e}\right)=M_{[1, i]^{c}}^{e}-M_{([1, i+1] \backslash\{i\})^{c}}^{e}-1=M_{[i+1, n+1]}^{e}-M_{\{i\} \cup[i+2, n+1]}^{e}-1$.

5.6. Comparison. As we explained above both $\mathcal{B}$ (with the $*$-crystal structure) and $\mathcal{B Z}^{e}$ are crystal which are isomorphic to $B(\infty)$. Therefore, as abstract crystals, they are isomorphic. In this subsection we will construct an explicit isomorphism form $\mathcal{B}$ to $\mathcal{B Z}^{e}$.

Following [BFZ], we introduce a notion of $K$-tableau for a Maya diagram $K \in \mathcal{M}_{n}^{\times}$.

Definition 5.6.1. Let $K=\left\{k_{1}<k_{2}<\cdots<k_{l}\right\} \in \mathcal{M}_{n}^{\times}$be a Maya diagram. For such $K$, we define a $K$-tableau as an upper-triangular matrix $C=\left(c_{p, q}\right)_{1 \leq p \leq q \leq l}$ with integer entries satisfying

$$
c_{p, p}=k_{p} \quad(1 \leq p \leq l)
$$

and the usual monotonicity conditions for semi-standard tableaux:

$$
c_{p, q} \leq c_{p, q+1}, \quad c_{p, q}<c_{p+1, q}
$$

For a giving $\mathbf{i}_{0}$-Lusztig datum $\mathbf{a}=\left(a_{i, j}\right) \in \mathcal{B}$, let $\mathbf{M}(\mathbf{a})=\left(M_{K}(\mathbf{a})\right)_{K \in \mathcal{M}_{n}^{\times}}$be a collection of integers defined by

$$
M_{K}(\mathbf{a})=-\sum_{j=1}^{l} \sum_{i=1}^{k_{j}-1} a_{i, k_{j}}+\min \left\{\sum_{1 \leq p<q \leq l} a_{c_{p, q}, c_{p, q}+(q-p)} \mid C=\left(c_{p, q}\right) \text { is a } K \text {-tableau }\right\}
$$

and denote the map $\mathbf{a} \mapsto \mathbf{M}(\mathbf{a})$ by $\Psi$.

Proposition 5.6.2 ([BFZ $])$. For any $\mathbf{a} \in \mathcal{B}, \Psi(\mathbf{a})=\mathbf{M}(\mathbf{a})$ is an e-BZ datum. Moreover $\Psi: \mathcal{B} \rightarrow \mathcal{B Z}^{e}$ is a bijection.

In this article, we prove the next theorem.

Theorem 5.6.3. The bijection $\Psi: \mathcal{B} \rightarrow \mathcal{B Z}^{e}$ is an isomorphism of crystals with respect to $*$-crystal structures.

To prove this theorem, it is enough to show the next two lemmas.

Lemma 5.6.4. For any $\mathbf{a} \in \mathcal{B}$, we have

$$
\operatorname{wt}(\mathbf{M}(\mathbf{a}))=\operatorname{wt}(\mathbf{a}), \quad \varepsilon_{i}^{*}(\mathbf{M}(\mathbf{a}))=\varepsilon_{i}^{*}(\mathbf{a}), \quad \varphi_{i}^{*}(\mathbf{M}(\mathbf{a}))=\varphi_{i}^{*}(\mathbf{a}) .
$$

Lemma 5.6.5. For any $\mathbf{a} \in \mathcal{B}$, we have

$$
\widetilde{e}_{i}^{*}(\mathbf{M}(\mathbf{a}))=\mathbf{M}\left(\widetilde{e}_{i}^{*} \mathbf{a}\right), \quad \widetilde{f}_{i}^{*}(\mathbf{M}(\mathbf{a}))=\mathbf{M}\left(\widetilde{f}_{i}^{*} \mathbf{a}\right) .
$$

Here we set $\mathbf{M}(0)=0$.

Proof of Lemma 5.6.4 Firstly let us compute $\operatorname{wt}(\mathbf{M}(\mathbf{a}))$. Since $\mathbf{M}(\mathbf{a})$ is an e-BZ datum we have

$$
\begin{aligned}
\operatorname{wt}(\mathbf{M}(\mathbf{a})) & =\sum_{i \in I} M_{[1, i]^{c}}(\mathbf{a}) \alpha_{i} \\
& =\sum_{i \in I} M_{[i+1, n+1]}(\mathbf{a}) \alpha_{i}
\end{aligned}
$$


For $K=[i+1, n+1]$, there exist a unique $K$-tableau

$$
C=\left(c_{p, q}\right)_{1 \leq p \leq q \leq n+1-i}=\left(\begin{array}{ccccc}
i+1 & i+1 & \cdots & \cdots & i+1 \\
& i+2 & i+2 & \cdots & i+2 \\
& & \ddots & \ddots & \vdots \\
& & & n & n \\
& & & & n+1
\end{array}\right) .
$$

That is, $c_{p, q}=i+p(1 \leq p \leq q \leq n+1-i)$. Therefore, for any $i \in I$, we have

$$
\begin{aligned}
M_{[i+1, n+1]} & =-\sum_{t=i+1}^{n+1} \sum_{s=1}^{t-1} a_{s, t}+\sum_{1 \leq p<q \leq n+1-i} a_{i+p, i+p+(q-p)} \\
& =-\sum_{t=i+1}^{n+1} \sum_{s=1}^{i} a_{s, t} \\
& =-m_{i}
\end{aligned}
$$

Here we set $m_{0}=m_{n+1}=0$. This equalities says that $\operatorname{wt}(\mathbf{M}(\mathbf{a}))=\operatorname{wt}(\mathbf{a})$.

Nextly let us calculate $\varepsilon_{i}^{*}(\mathbf{M}(\mathbf{a}))$. We have

$$
\begin{aligned}
\varepsilon_{i}^{*}(\mathbf{M}(\mathbf{a})) & =\varepsilon_{i}\left(\mathbf{M}(\mathbf{a})^{*}\right) \\
& =-M_{[i+1, n+1]}(\mathbf{a})-M_{\{i\} \cup[i+2, n+1]}(\mathbf{a})+M_{[i+2, n+1]}(\mathbf{a})+M_{[i, n+1]}(\mathbf{a}) .
\end{aligned}
$$

From the proof of the first formula we already know

$$
M_{[k+1, n+1]}(\mathbf{a})=-\sum_{t=k+1}^{n+1} \sum_{s=1}^{k} a_{s, t} \quad(k=i-1, i, i+1) .
$$

For $K=\{i\} \cup[i+2, n+1]$, the set of all $K$-tableaux is given by $\left\{C^{(r)}\right\}_{1 \leq r \leq n+1-i}$ where

$$
C^{(r)}=\left(c_{p, q}^{(r)}\right)_{1 \leq p \leq q \leq n+1-i}=\left(\begin{array}{ccccc}
i & c_{1,2}^{(r)} & \cdots & \cdots & c_{1, n+1-i}^{(r)} \\
& i+2 & i+2 & \cdots & i+2 \\
& & \ddots & \ddots & \vdots \\
& & & n & n \\
& & & & n+1
\end{array}\right)
$$

and

$$
c_{1, q}^{(r)}= \begin{cases}i & (2 \leq q \leq r) \\ i+1 & (r<q \leq n+1-i)\end{cases}
$$

Since

$$
\sum_{q=2}^{n+1-i} a_{c_{1, q}^{(r)}, c_{1, q}^{(r)}+(q-1)}=\sum_{q=i+1}^{i+r-1} a_{i, q}+\sum_{q=i+r+1}^{n+1} a_{i+1, q}
$$


we have

$$
\begin{aligned}
M_{\{i\} \cup[i+2, n+1]}(\mathbf{a})= & -\sum_{s=1}^{i-1} a_{s, i}-\sum_{t=i+2}^{n+1} \sum_{s=1}^{t-1} a_{s, t}+\min _{1 \leq r \leq n+1-i}\left\{\sum_{1 \leq p<q \leq l} a_{c_{p, q}^{(r)}, c_{p, q}^{(r)}+(q-p)}\right\} \\
= & -\sum_{s=1}^{i-1} a_{s, i}-\sum_{t=i+2}^{n+1} \sum_{s=1}^{t-1} a_{s, t}+\sum_{q=i+3}^{n+1} \sum_{p=i+2}^{q-1} a_{p, q} \\
& +\min _{1 \leq r \leq n+1-i}\left\{\sum_{q=2}^{n+1-i} a_{c_{1, q}^{(r)}, c_{1, q}^{(r)}+(q-1)}\right\} \\
= & -\sum_{s=1}^{i-1} a_{s, i}-\sum_{t=i+2}^{n+1} \sum_{s=1}^{i+1} a_{s, t}+\min _{1 \leq r \leq n+1-i}\left\{\sum_{q=i+1}^{i+r-1} a_{i, q}+\sum_{q=i+r+1}^{n+1} a_{i+1, q}\right\} .
\end{aligned}
$$

Putting together all formulas, we have

$$
\begin{aligned}
\varepsilon_{i}^{*}(\mathbf{M}(\mathbf{a}))= & \sum_{t=i+1}^{n+1} \sum_{s=1}^{i} a_{s, t}-\sum_{t=i}^{n+1} \sum_{s=1}^{i-1} a_{s, t}-\sum_{t=i+2}^{n+1} \sum_{s=1}^{i+1} a_{s, t}+\sum_{s=1}^{i-1} a_{s, i}+\sum_{t=i+2}^{n+1} \sum_{s=1}^{i+1} a_{s, t} \\
& -\min _{1 \leq r \leq n+1-i}\left\{\sum_{q=i+1}^{i+r-1} a_{i, q}+\sum_{q=i+r+1}^{n+1} a_{i+1, q}\right\} \\
& =\sum_{t=i+1}^{n+1} a_{i, t}-\min _{1 \leq r \leq n+1-i}\left\{\sum_{q=i+1}^{i+r-1} a_{i, q}+\sum_{q=i+r+1}^{n+1} a_{i+1, q}\right\} \\
& =\max _{1 \leq r \leq n+1-i}\left\{\sum_{q=i+r}^{n+1} a_{i, q}-\sum_{q=i+r+1}^{n+1} a_{i+1, q}\right\} \\
& =\max _{1 \leq r \leq n+1-i}\left\{A_{i-1+r}^{*(i)}(\mathbf{a})\right\} \\
& =\varepsilon_{i}^{*}(\mathbf{a}) .
\end{aligned}
$$

Finally let us prove $\varphi_{i}^{*}(\mathbf{M}(\mathbf{a}))=\varphi_{i}^{*}(\mathbf{a})$. But it is clear by the first and second formulas.

We can prove Lemma 5.6.5 by direct calculation. But in this article we give another proof by using a Lagrangian constriction of $B(\infty)$, which we will explain later. (See Subsection 7.3.)

\section{Quivers OF TYPE $A_{n}$}

6.1. Quivers and their representations. Let $(I, H)$ be the double quiver of type $A_{n}$. Here $I=\{1,2, \cdots, n\}$ is the set of vertices and $H$ is the set of arrows. If $\tau \in H$ is the arrow from $i$ to $j$, we denote $\operatorname{out}(\tau)=i$ and $\operatorname{in}(\tau)=j$. For that $\tau \in H$, let $\bar{\tau}$ be the arrow from $j$ to $i$. The map $\tau \mapsto \bar{\tau}$ defines an involution of $H$. An orientation $\Omega$ is a subset of $H$ such that $\Omega \cap \bar{\Omega}=\phi$ and $\Omega \cup \bar{\Omega}=H$. Then $(I, \Omega)$ is a Dynkin quiver of type $A_{n}$.

Let $\mathbf{V}=(V, B)$ be a representation of the quiver $(I, \Omega)$. Here $V=\oplus_{i \in I} V_{i}$ be a finite dimensional $I$-graded complex vector space with the dimension vector $\operatorname{dim} V=$ $\left(\operatorname{dim}_{\mathbb{C}} V_{i}\right)_{i \in I} \in \mathbb{Z}_{\geq 0}^{I}$, and $B=\left(B_{\tau}\right)_{\tau \in \Omega}$ is a collection of linear maps $B_{\tau}: V_{\text {out }(\tau)} \rightarrow V_{\operatorname{in}(\tau)}$. We denote by $M \Omega$ the category of representations of the quiver $(I, \Omega)$. Let $\mathbf{V}=(V, B)$, $\mathbf{V}^{\prime}=\left(V^{\prime}, B^{\prime}\right) \in M \Omega$. A morphism $\phi=\left(\phi_{i}\right)_{i \in I}$ form $\mathbf{V}$ to $\mathbf{V}^{\prime}$ is a collection of linear 
maps $\phi_{i}: V_{i} \rightarrow V_{i}^{\prime}$ such that $\phi_{\operatorname{in}(\tau)} B_{\tau}=B_{\tau}^{\prime} \phi_{\text {out }(\tau)}$ for any $\tau \in \Omega$. It is well-known that $M \Omega$ is a Krull-Schmidt category. That is, any object in $M \Omega$ has a unique indecomposable decomposition. For $i \in I$ let $\mathbf{e}(i ; \Omega)$ be a representation of $(I, \Omega)$ defined by $V_{i}=\mathbb{C}$ and $V_{j}=0$ for $j \neq i$. This is simple and any simple representation isomorphic to $\mathbf{e}(i ; \Omega)$, for a unique $i$.

Assume that $i \in I$ is a sink (resp. a source) of an orientation $\Omega$. That is, there is no arrow $\tau \in \Omega$ such that $\operatorname{out}(\tau)=i$ (resp. $\operatorname{in}(\tau)=i$ ). We denote by $\operatorname{sink}(\Omega)$ (resp. source $(\Omega)$ ) the set of all sink (resp. source) vertices. Let $s_{i} \Omega$ be the orientation obtained from $\Omega$ by reversing each arrow $\tau$ such that $\operatorname{in}(\tau)=i(\operatorname{resp} . \operatorname{out}(\tau)=i)$.

Definition 6.1.1. Fix an orientation $\Omega$. A reduced word $\mathbf{i}=\left(i_{1}, \cdots, i_{N}\right)$ of $w_{0}$ is said to be adapted to $\Omega$ if $i_{k}$ is a sink of $\Omega_{k}=s_{i_{k-1}} \cdots s_{1} \Omega$ for $1 \leq k \leq N$.

For a representation of a quiver $\mathbf{V}=(V, B)$, we set $\operatorname{dim} \mathbf{V}=\operatorname{dim} V$. From now on, we identify the dimension vector $\operatorname{dim} V=\left(\operatorname{dim}_{\mathbb{C}} V_{i}\right)_{i \in I} \in \mathbb{Z}_{\geq 0}^{I}$ with an element of $Q_{+}=$ $\oplus_{i \in I} \mathbb{Z}_{\geq 0} \alpha_{i}$ by

$$
\left(\operatorname{dim}_{\mathbb{C}} V_{i}\right)_{i \in I} \mapsto \sum_{i \in I}\left(\operatorname{dim}_{\mathbb{C}} V_{i}\right) \alpha_{i} .
$$

Proposition 6.1.2. [L1] (1) For a giving orientation $\Omega$, there exist a reduced word $\mathbf{i}$ of $w_{0}$ adapted to $\Omega$.

(2) For each $\beta \in \Delta^{+}$, there is a unique indecomposable representation (up to isomorphism $) \mathbf{e}(\beta ; \Omega)$ such that $\operatorname{dim} \mathbf{e}(\beta ; \Omega)=\beta$. Moreover any indecomposable representation is isomorphic to $\mathbf{e}(\beta ; \Omega)$ for a unique $\beta$ (Gabriel's theorem).

(3) If $\beta>_{\mathbf{i}} \beta^{\prime}$, we have $\operatorname{Hom}_{M \Omega}\left(\mathbf{e}(\beta ; \Omega), \mathbf{e}\left(\beta^{\prime} ; \Omega\right)\right)=0$.

6.2. Orientations arising from Maya diagrams. Any Maya diagram $K \in \mathcal{M}_{n}^{\times}$can be written as a disjoint union of intervals

$$
\begin{gathered}
K=\left[s_{1}+1, t_{1}\right] \sqcup\left[s_{2}+1, t_{2}\right] \sqcup \cdots \sqcup\left[s_{l}+1, t_{l}\right] \\
\left(0 \leq s_{1}<t_{1}<s_{2}<t_{2}<\cdots<s_{l}<t_{l} \leq n+1\right) ;
\end{gathered}
$$

the interval $K_{m}=\left[s_{m}+1, t_{m}\right](1 \leq m \leq l)$ will be called the $m$-th component of $K$. Define two subsets out $(K)$ and $\operatorname{in}(K)$ of $[1, n]$ by

$$
\operatorname{out}(K)=\left\{t_{m} \mid 1 \leq m \leq l\right\} \cap[1, n], \quad \operatorname{in}(K)=\left\{s_{m} \mid 1 \leq m \leq l\right\} \cap[1, n] .
$$

We remark that $\operatorname{out}(K) \cap \operatorname{in}(K)=\phi$. Introduce two subsets $I_{t}$ and $I_{s}$ as follows:

$$
\begin{gathered}
I_{t}= \begin{cases}\operatorname{out}(K) \cup\{1, n\} & \left(s_{1} \geq 2, t_{l}=n+1\right), \\
\operatorname{out}(K) \cup\{1\} & \left(s_{1} \geq 2, t_{l} \leq n\right), \\
\operatorname{out}(K) \cup\{n\} & \left(s_{1} \leq 1, t_{l}=n+1\right), \\
\operatorname{out}(K) & \left(s_{1} \leq 1, t \leq n\right) .\end{cases} \\
I_{s}= \begin{cases}\operatorname{in}(K) \cup\{1, n\} & \left(s_{1}=0, t_{l} \leq n-1\right), \\
\operatorname{in}(K) \cup\{1\} & \left(s_{1}=0, t_{l} \geq n\right), \\
\operatorname{in}(K) \cup\{n\} & \left(s_{1} \geq 1, t_{l} \leq n-1\right), \\
\operatorname{in}(K) & \left(s_{1} \geq 1, t_{l} \geq n\right)\end{cases}
\end{gathered}
$$

Definition 6.2.1. (1) In the above setting, there exist a unique orientation $\Omega(K)$ so that source $(\Omega(K))=I_{t}$ and $\operatorname{sink}(\Omega(K))=I_{s}$. We call $\Omega(K)$ the orientation arising from $a$ Maya diagram $K \in \mathcal{M}_{n}^{\times}$.

(2) Let $s_{K}=\min \{k \mid k \notin K\}$ and $t_{K}=\max \{k \mid k \in K\}$. Define $\beta_{K} \in \Delta^{+} \cup\{0\}$ by

$$
\beta_{K}= \begin{cases}\alpha_{s_{K}}+\alpha_{s_{K}+1}+\cdots+\alpha_{t_{K}-1} & \left(s_{K}<t_{K}\right), \\ 0 & \text { (otherwise) }\end{cases}
$$


and we call it the characterizing positive root of a Maya diagram $\mathrm{K}$.

Remark . (1) In general, we have

$$
\operatorname{out}(K) \subset \operatorname{source}(\Omega(K)), \quad \operatorname{in}(K) \subset \operatorname{sink}(\Omega(K)) .
$$

(2) The characterizing positive root $\beta_{K}=0$ if and only if $K=\left[1, t_{1}\right]$ for some $1 \leq t_{1} \leq n$.

Example 6.2.2. Let $n=17$ and $K=[3,4] \sqcup[7,8] \sqcup[10,12] \sqcup[14,15]$. Then we have

$$
\operatorname{out}(K)=\{4,8,12,15\}, \quad \operatorname{in}(K)=\{2,6,9,13\} .
$$

Since $s_{1}=2$ and $t_{l}=t_{4}=15$, we have

$$
I_{t}=\operatorname{out}(K) \cup\{1\}=\{1,4,8,12,15\}, \quad I_{s}=\operatorname{in}(K) \cup\{17\}=\{2,6,9,13,17\} .
$$

In this case, the orientation $\Omega(K)$ is given as follows:

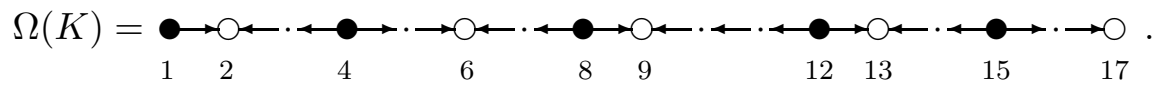

Here $\circ$ is a sink and $\bullet$ is a source

Since $s_{K}=1$ and $t_{K}=15$, the characterizing positive root $\beta_{K}$ is given by

$$
\beta_{K}=\sum_{i=1}^{14} \alpha_{i}
$$

6.3. From Lusztig data to $e-\mathbf{B Z}$ data. Let $\mathbf{i}$ be a reduced word adapted to the orientation $\Omega(K)$ and consider the set of all $\mathbf{i}$-Lusztig data

$$
\mathcal{B}^{\mathbf{i}}=\left\{\mathbf{a}^{\mathbf{i}}=\left(a_{i, j}^{\mathbf{i}}\right)_{(i, j) \in \Pi} \mid a_{i, j}^{\mathbf{i}} \in \mathbb{Z}_{\geq 0}\right\} .
$$

Recall the identification $\Delta^{+} \stackrel{\sim}{\rightarrow} \Pi$ (see Subsection 2.4) and denote the image of $\beta \in \Delta^{+}$ by $\left(i_{\beta}, j_{\beta}\right) \in \Pi$. Set $\mathbf{e}\left(\left(i_{\beta}, j_{\beta}\right) ; \Omega(K)\right)=\mathbf{e}(\beta ; \Omega(K))$. Then, for each $\mathbf{V} \in M \Omega(K)$, there is a unique $\mathbf{a}^{\mathbf{i}} \in \mathcal{B}^{\mathbf{i}}$ such that $\mathbf{V}$ is isomorphic to $\mathbf{V}\left(\mathbf{a}^{\mathbf{i}}\right)$. Here

$$
\mathbf{V}\left(\mathbf{a}^{\mathbf{i}}\right)=\underset{(i, j) \in \Pi}{\oplus} \mathbf{e}((i, j) ; \Omega(K))^{\oplus a_{i, j}^{\mathbf{i}}} .
$$

We introduce a non-positive integer

$$
M_{K}\left(\mathbf{V}\left(\mathbf{a}^{\mathbf{i}}\right)\right)=-\operatorname{dim}_{\mathbb{C}} \operatorname{Hom}_{M \Omega(K)}\left(\mathbf{V}\left(\mathbf{a}^{\mathbf{i}}\right), \mathbf{e}\left(\beta_{K} ; \Omega(K)\right)\right) .
$$

Lemma 6.3.1. (1) We have

$$
M_{K}\left(\mathbf{V}\left(\mathbf{a}^{\mathbf{i}}\right)\right)=-\sum_{(i, j) \in \Pi ; i \notin K, j \in K} a_{i, j}^{\mathbf{i}} .
$$

(2) Denote $\mathbf{V}\left(\mathbf{a}^{\mathbf{i}}\right)=\left(\oplus_{i} V_{i},\left(B_{\tau}\right)_{\tau \in \Omega(K)}\right)$. Then we have

$$
\sum_{(i, j) \in \Pi ; i \notin K, j \in K} a_{i, j}^{\mathbf{i}}=\operatorname{dim}_{\mathbb{C}} \operatorname{Coker}\left(\underset{k \in \operatorname{out}(K)}{\oplus} V_{k} \stackrel{\oplus B_{q}}{\longrightarrow} \underset{l \in \operatorname{in}(K)}{\oplus} V_{l}\right) .
$$

Here $\sigma$ is a path in $\Omega(K)$ form some $k \in \operatorname{out}(K)$ to some $l \in \operatorname{in}(K)$

The proof of this lemma will be given in the next subsection.

The next proposition is a easy consequence of the results of Berenstein, Fomin and Zelevinsky [BFZ]. 
Proposition 6.3.2. Let $\mathbf{i}$ be a reduced word adapted to the orientation $\Omega(K)$ and $\mathbf{a} \in \mathcal{B}$ an $\mathbf{i}_{0}$-Lusztig datum. Set $\mathbf{a}^{\mathbf{i}}=R_{\mathbf{i}_{0}}^{\mathbf{i}}(\mathbf{a})$. Here $R_{\mathbf{i}_{0}}^{\mathbf{i}}$ is the transition map from $\mathbf{i}_{0}$ to $\mathbf{i}$. Then we have

$$
M_{K}(\mathbf{a})=-\sum_{(i, j) \in \Pi ; i \notin K, j \in K} a_{i, j}^{\mathbf{i}} .
$$

Here $\mathbf{M}(\mathbf{a})=\left(M_{K}(\mathbf{a})\right)_{K \in \mathcal{M}_{n}^{\times}}$is the e-BZ datum defined in 5.6.

Combining the above results, we have the following corollary:

Corollary 6.3.3. In the above setting, we have

$$
M_{K}(\mathbf{a})=M_{K}\left(\mathbf{V}\left(\mathbf{a}^{\mathbf{i}}\right)\right)=-\operatorname{dim}_{\mathbb{C}} \operatorname{Coker}\left(\underset{k \in \operatorname{out}(K)}{\oplus} V_{k} \stackrel{\oplus B_{q}}{\longrightarrow} \underset{l \in \operatorname{in}(K)}{\oplus} V_{l}\right) .
$$

6.4. Proof of Lemma 6.3.1. Let us prove the formula (1). It is enough to show that

$$
\operatorname{dim}_{\mathbb{C}} \operatorname{Hom}_{M \Omega(K)}\left(\mathbf{e}((i, j) ; \Omega(K)), \mathbf{e}\left(\beta_{K} ; \Omega(K)\right)\right)= \begin{cases}1 & (i \notin K \text { and } j \in K), \\ 0 & \text { (otherwise). }\end{cases}
$$

Let us denote $\mathbf{e}((i, j) ; \Omega(K))=\left(\oplus V_{k}^{\prime},\left(B_{\tau}^{\prime}\right)\right)$ and $\mathbf{e}\left(\beta_{K} ; \Omega(K)\right)=\left(\oplus V_{k}^{\prime \prime},\left(B_{\tau}^{\prime \prime}\right)\right)$. Then

$$
V_{k}^{\prime}=\left\{\begin{array}{ll}
\mathbb{C} & (i \leq k \leq j-1), \\
0 & \text { (otherwise) }
\end{array} \quad \text { and } \quad V_{k}^{\prime \prime}= \begin{cases}\mathbb{C} & \left(s_{K} \leq k \leq t_{K}-1\right), \\
0 & \text { (otherwise) }\end{cases}\right.
$$

Firstly assume $i \notin K$ and $j \in K$. Since our quiver is of type $A_{n}$, the left hand side of (6.4.1) is less than 1. So it is enough to show that there is a non-trivial morphism form $\left(\oplus V_{k}^{\prime},\left(B_{\tau}^{\prime}\right)\right)$ to $\left(\oplus V_{k}^{\prime \prime},\left(B_{\tau}^{\prime \prime}\right)\right)$. By the assumption we have $s_{K} \leq i<j \leq t_{K}$. Therefore we can define a linear map $\psi=\left(\psi_{k}\right): \oplus V_{k}^{\prime} \rightarrow \oplus V_{k}^{\prime \prime}$ by

$$
\psi_{k}= \begin{cases}\operatorname{id}_{\mathbb{C}} & (i \leq k \leq j-1) \\ 0 & \text { (otherwise) }\end{cases}
$$

and it is easy to check that the above map is a non-trivial morphism of $M \Omega(K)$.

Secondly let us consider the case that $i \in K$ or $j \notin K$. The goal is to prove

$$
\operatorname{Hom}_{M \Omega(K)}\left(\left(\oplus V_{k}^{\prime},\left(B_{\tau}^{\prime}\right)\right),\left(\oplus V_{k}^{\prime \prime},\left(B_{\tau}^{\prime \prime}\right)\right)\right)=0 .
$$

Assume $i \in K$ and $s_{K}<i$. Then $i \geq 2, i+1 \leq t_{K}$ and there is an arrow $\tau_{1}$ form $i$ to $i-1$ in $\Omega(K)$. Let $\psi=\left(\psi_{k}\right) \in \operatorname{Hom}_{M \Omega(K)}\left(\left(\oplus V_{k}^{\prime},\left(B_{\tau}^{\prime}\right)\right),\left(\oplus V_{k}^{\prime \prime},\left(B_{\tau}^{\prime \prime}\right)\right)\right)$. Since $V_{i-1}^{\prime}=0$, we have $B_{\tau_{1}}^{\prime \prime} \psi_{i}=\psi_{i-1} B_{\tau_{1}}^{\prime}=0$. On the other hand, $B_{\tau_{1}}^{\prime \prime} \neq 0$ because $s_{K} \leq i-1$. Therefore we have $\psi_{i}=0$.

If $j=i+1$ or $t_{K}=i+1$, it means that the left hand side of (6.4.2) is equal to zero. So we may assume $j>i+1$ and $t_{K}>i+1$. However one can show that $\psi_{i+1}=0$. Indeed, if there is an arrow $\tau_{2}$ form $i$ to $i+1$ in $\Omega(K)$, then we have $\psi_{i+1} B_{\tau_{2}}^{\prime}=B_{\tau_{2}}^{\prime \prime} \psi_{i}$ with non-trivial $B_{\tau_{2}}^{\prime}$ and $B_{\tau_{2}}^{\prime \prime}$. Since $\psi_{i}=0$, we have $\psi_{i+1}=0$. On the other hand, if there is an arrow $\tau_{2}$ form $i+1$ to $i$ in $\Omega(K)$, then we have $\psi_{i} B_{\tau_{2}}^{\prime}=B_{\tau_{2}}^{\prime \prime} \psi_{i+1}$. Then we also have $\psi_{i+1}=0$. By repeating this method, we have $\psi_{k}=0$ for any $k \in I$.

For the other cases, we can show (6.4.2) by similar way.

We will give a proof of (2). Since $\mathbf{V}\left(\mathbf{a}^{\mathbf{i}}\right)=\oplus_{(i, j) \in \Pi} \mathbf{e}((i, j) ; \Omega(K))^{\oplus a_{i, j}^{\mathbf{i}} \text { is the indecom- }}$ posable decomposition, it is enough to prove that

$$
\operatorname{dim}_{\mathbb{C}} \operatorname{Coker}\left(\underset{k \in \operatorname{out}(K)}{\oplus} V_{k}^{\prime} \stackrel{\oplus B_{\sigma}^{\prime}}{\longrightarrow} \underset{l \in \operatorname{in}(K)}{\oplus} V_{l}^{\prime}\right)= \begin{cases}1 & (i \notin K \text { and } j \in K), \\ 0 & \text { (otherwise). }\end{cases}
$$

Recall the decomposition of $K$ :

$$
K=K_{1} \sqcup \cdots \sqcup K_{l}, \quad \text { where } K_{m}=\left[s_{m}+1, t_{m}\right] \quad(1 \leq m \leq l) .
$$


Firstly assume $i \notin K$ and $j \in K$. More precisely, we assume $t_{u-1}<i<s_{u}+1$ and $s_{v}+1 \leq j \leq t_{v}$ with $u \leq v$. Let $\sigma\left(t_{m-1} \rightarrow s_{m}\right)\left(\right.$ resp. $\left.\sigma\left(s_{m} \leftarrow t_{m}\right)\right)$ be the path form $t_{m-1}$ to $s_{m}$ (resp. from $t_{m}$ to $s_{m}$ ) in $\Omega(K)$. Then, by the definition, we have

$$
B_{\sigma\left(t_{m-1} \rightarrow s_{m}\right)}^{\prime}=\left\{\begin{array}{ll}
\operatorname{id}_{\mathbb{C}} & (u+1 \leq m \leq v), \\
0 & \text { (otherwise) },
\end{array} B_{\sigma\left(s_{m} \leftarrow t_{m}\right)}^{\prime}= \begin{cases}\operatorname{id}_{\mathbb{C}} & (u \leq m \leq v-1), \\
0 & \text { (otherwise) } .\end{cases}\right.
$$

Therefore we have

$$
\begin{aligned}
& \operatorname{dim}_{\mathbb{C}} \operatorname{Coker}\left(\underset{k \in \operatorname{out}(K)}{\oplus} V_{k}^{\prime} \stackrel{\oplus B_{a}^{\prime}}{\left.\underset{l \in \operatorname{in}(K)}{\oplus} V_{l}^{\prime}\right)}=\operatorname{dim}_{\mathbb{C}} \operatorname{Coker}\left(\underset{m=u}{\oplus-1} V_{t_{m}}^{\prime} \longrightarrow \underset{m=u}{\oplus} V_{s_{m}}^{\prime}\right)\right. \\
&=\operatorname{dim}_{\mathbb{C}} \operatorname{Coker}\left(\mathbb{C}^{v-u-1} \hookrightarrow \mathbb{C}^{v-u}\right) \\
&=1 .
\end{aligned}
$$

Secondly assume $i, j \in K$. Then there exist $u$ and $v$ with $u \leq v$ such that $i \in K_{u}$ and $j \in K_{v}$. In this case, we have

$$
B_{\sigma\left(t_{m-1} \rightarrow s_{m}\right)}^{\prime}=\left\{\begin{array}{ll}
\operatorname{id}_{\mathbb{C}} & (u+1 \leq m \leq v), \\
0 & \text { (otherwise) },
\end{array} B_{\sigma\left(s_{m} \leftarrow t_{m}\right)}^{\prime}= \begin{cases}\operatorname{id}_{\mathbb{C}} & (u+1 \leq m \leq v-1), \\
0 & \text { (otherwise) } .\end{cases}\right.
$$

Therefore we have

$$
\begin{aligned}
\operatorname{dim}_{\mathbb{C}} \operatorname{Coker}\left(\underset{k \in \operatorname{Out}(K)}{\oplus} V_{k}^{\prime} \stackrel{\oplus B_{g}^{\prime}}{\rightarrow} \underset{l \in \operatorname{in}(K)}{\oplus} V_{l}^{\prime}\right) & =\operatorname{dim}_{\mathbb{C}} \operatorname{Coker}\left(\underset{m=u}{\oplus-1} V_{t_{m}}^{\prime} \longrightarrow \underset{m=u+1}{\oplus} V_{s_{m}}^{\prime}\right) \\
& =\operatorname{dim}_{\mathbb{C}} \operatorname{Coker}\left(\mathbb{C}^{v-u-1} \stackrel{\sim}{\rightarrow} \mathbb{C}^{v-u-1}\right) \\
& =0 .
\end{aligned}
$$

For the other cases, we can prove that the left hand side of (6.4.3) equals to zero by similar arguments. Thus, the lemma is proved.

\section{LAGRANGIAN CONSTRUCTION OF CRYSTAL BASIS}

7.1. Varieties associated to quivers. For $\nu \in Q_{+}$, let $\mathcal{V}_{\nu}$ be the category of $I$-graded complex vector spaces $V$ with $\operatorname{dim} V=\nu$. For $V=\oplus_{i \in I} V_{i} \in \mathcal{V}_{\nu}$, introduce two complex vector spaces

$$
E_{V, \Omega}=\underset{\tau \in \Omega}{\oplus} \operatorname{Hom}_{\mathbb{C}}\left(V_{\text {out }(\tau)}, V_{\operatorname{in}(\tau)}\right), \quad X_{V}=\underset{\tau \in H}{\oplus} \operatorname{Hom}_{\mathbb{C}}\left(V_{\text {out }(\tau)}, V_{\operatorname{in}(\tau)}\right) .
$$

An element of $E_{V, \Omega}$ or $X_{V}$ will be denoted by $B=\left(B_{\tau}\right)$ where $B_{\tau} \in \operatorname{Hom}_{\mathbb{C}}\left(V_{\text {out }(\tau)}, V_{\operatorname{in}(\tau)}\right)$. Define a symplectic form $\omega$ on $X_{V}$ by

$$
\omega\left(B, B^{\prime}\right)=\sum_{\tau \in H} \varepsilon(\tau) \operatorname{tr}\left(B_{\bar{\tau}} B_{\tau}^{\prime}\right)
$$

where $\varepsilon(\tau)=1$ for $\tau \in \Omega$ and $\varepsilon(\tau)=-1$ for $\tau \in \bar{\Omega}$. We regard $X_{V}$ as the cotangent bundle $T^{*} E_{V, \Omega}$ of $E_{V, \Omega}$ via the symplectic form $\omega$.

The group $G_{V}=\prod_{i \in I} G L\left(V_{i}\right)$ acts on $E_{V, \Omega}$ and $X_{V}$ by

$$
G_{V} \ni g=\left(g_{i}\right):\left(B_{\tau}\right) \mapsto\left(g_{\text {in }(\tau)} B_{\tau} g_{\text {out }(\tau)}^{-1}\right) .
$$

Since the action of $G_{V}$ on $X_{V}$ preserves the symplectic form $\omega$, we can consider the corresponding moment map $\mu: X_{V} \rightarrow\left(\mathfrak{g}_{V}\right)^{*} \cong \mathfrak{g}_{V}$. Here $\mathfrak{g}_{V}=$ Lie $G_{V}$ and we identify $\mathfrak{g}_{V}$ with its dual via the Killing form. Set

$$
\Lambda_{V}=\mu^{-1}(0) .
$$


It is known that $\Lambda_{V}$ is a $G_{V^{-}}$-invariant closed Lagrangian subvariety of $X_{V}$. It is clear that, for $V, V^{\prime} \in \mathcal{V}_{\nu}$, there are natural isomorphisms $V \cong V^{\prime}, X_{V} \cong X_{V^{\prime}}$ and $\Lambda_{V} \cong \Lambda_{V^{\prime}}$. Hence we denote them $V(\nu), X(\nu)$ and $\Lambda(\nu)$, respectively.

Let $\operatorname{Irr} \Lambda(\nu)$ be a set of all irreducible components of $\Lambda(\nu)$. Since our quiver is of type $A_{n}$, there is a bijection from the set of all $G_{V(\nu)}$-orbits in $E_{V(\nu), \Omega}$ to $\operatorname{Irr} \Lambda(\nu)$ defined by $\mathcal{O} \mapsto \overline{T_{\mathcal{O}}^{*} E_{V(\nu), \Omega}}$. We remark that $E_{V(\nu), \Omega}$ has finitely many $G_{V(\nu)}$-orbits because our quiver is of type $A_{n}$.

For $B \in E_{V(\nu), \Omega}$, a pair $\mathbf{V}=(V(\nu), B)$ is nothing but a representation of a quiver $(I, \Omega)$ with a dimension vector $\nu$. Moreover there is a natural one to one correspondence between isomorphism classes of representations of a quiver $(I, \Omega)$ with a dimension vector $\nu$ and $G_{V(\nu)}$-orbits in $E_{V(\nu), \Omega}$.

Let $\Omega$ be an orientation and $\mathbf{i}$ a reduced word adapted to $\Omega$. As we mentioned before, for each $\mathbf{V}=(V(\nu), B) \in M \Omega$, there is a unique $\mathbf{i}$-Lusztig datum $\mathbf{a}^{\mathbf{i}} \in \mathcal{B}^{\mathbf{i}}$ such that $\mathbf{V}$ is isomorphic to

$$
\mathbf{V}\left(\mathbf{a}^{\mathbf{i}}\right)=\underset{(i, j) \in \Pi}{\oplus} \mathbf{e}((i, j) ; \Omega)^{\oplus a_{i, j}^{\mathbf{i}}}
$$

Let $\mathcal{O}_{\mathbf{a}^{\mathbf{i}}}$ be the $G_{V(\nu)}$-orbit of $E_{V(\nu), \Omega}$ through $\mathbf{V}\left(\mathbf{a}^{\mathbf{i}}\right)$. Denote $\Lambda_{\mathbf{a}^{\mathbf{i}}}=\overline{T_{\mathcal{O}_{\mathbf{a}^{\mathbf{i}}}^{*}} E_{V(\nu), \Omega}}$. Then we have a bijection $\Psi_{\mathbf{i}}: \mathcal{B}^{\mathbf{i}} \stackrel{\sim}{\rightarrow} \bigsqcup_{\nu \in Q^{-}} \operatorname{Irr} \Lambda(\nu)$ defined by $\mathbf{a}^{\mathbf{i}} \mapsto \Lambda_{\mathbf{a}^{\mathbf{i}}}$. Especially, consider the following special orientation

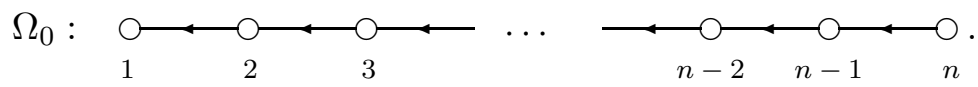

Then the lexicographically minimal reduced word $\mathbf{i}_{0}$ is adapted to $\Omega_{0}$. For $\mathbf{a} \in \mathcal{B}$, let $\mathcal{O}_{\mathbf{a}}$ be the corresponding orbit in $E_{V(\nu), \Omega_{0}}$ and $\Lambda_{\mathbf{a}}=\overline{T_{\mathcal{O}_{\mathbf{a}}}^{*} E_{V(\nu), \Omega_{0}}}$. We remark that

$$
\Lambda_{\mathbf{a}}=\Lambda_{\mathbf{a}^{\mathbf{i}}}
$$

where $\mathbf{i}$ is an arbitrarily reduced word and $\mathbf{a}^{\mathbf{i}}=R_{\mathbf{i}_{0}}^{\mathbf{i}}(\mathbf{a})$.

Remark . It seems to us that the formula (7.1.1) is known for experts. However the proof of it was not appeared until recently. A detailed proof was firstly given by Kimura in his Master thesis [Kim] (see Appendix A, in detail).

On the other hand, in 2010, Baumann and Kamnitzer give another explicit proof of it by using representation theory of preprojective algebra (see [BK]).

For $B \in X(\nu)$ we set

$$
M_{K}(B)=-\operatorname{dim}_{\mathbb{C}} \operatorname{Coker}\left(\underset{k \in \operatorname{out}(K)}{\oplus} V(\nu)_{k} \stackrel{\oplus B_{\sigma}}{\longrightarrow} \underset{l \in \operatorname{in}(K)}{\oplus} V(\nu)_{l}\right)
$$

and for $\Lambda \in \operatorname{Irr} \Lambda(\nu)$ define

$$
M_{K}(\Lambda)=M_{K}(B)
$$

by taking a generic point $B$ of $\Lambda$. By Corollary 6.3.3, we immediately have the following statement.

Corollary 7.1.1. Recall the setting of Corollary 6.3.3; let $\mathbf{i}$ be a reduced word which is adapted to the orientation $\Omega(K)$ and $\mathbf{a} \in \mathcal{B}$ an $\mathbf{i}_{0}$-Lusztig datum. Set $\mathbf{a}^{\mathbf{i}}=R_{\mathbf{i}_{0}}^{\mathbf{i}}(\mathbf{a})$. Then we have

$$
M_{K}(\mathbf{a})=M_{K}\left(\mathbf{V}\left(\mathbf{a}^{\mathbf{i}}\right)\right)=M_{K}\left(\Lambda_{\mathbf{a}^{\mathbf{i}}}\right) .
$$

Combining the above corollary with (7.1.1), we have

$$
M_{K}(\mathbf{a})=M_{K}\left(\Lambda_{\mathbf{a}}\right) \text {. }
$$


7.2. Lagrangian construction of $B(\infty)$. In this subsection we will give a review of Lagrangian construction of $B(\infty)$ following [KS].

Let $\nu, \nu^{\prime}, \bar{\nu} \in Q_{+}$with $\nu=\nu^{\prime}+\bar{\nu}$. Consider a diagram

$$
\Lambda\left(\nu^{\prime}\right) \times \Lambda(\bar{\nu}) \stackrel{q_{1}}{\longleftarrow} \Lambda\left(\nu^{\prime}, \bar{\nu}\right) \stackrel{q_{2}}{\longrightarrow} \Lambda(\nu) .
$$

Here $\Lambda\left(\nu^{\prime}, \bar{\nu}\right)$ is a variety of $\left(B, \phi^{\prime}, \bar{\phi}\right)$, where $B \in \Lambda(\nu)$ and $\phi^{\prime}=\left(\phi_{i}^{\prime}\right), \bar{\phi}=\left(\bar{\phi}_{i}\right)$ give an exact sequence

$$
0 \longrightarrow V\left(\nu^{\prime}\right)_{i} \stackrel{\phi_{i}^{\prime}}{\longrightarrow} V(\nu) \stackrel{\bar{\phi}_{i}}{\longrightarrow} V(\bar{\nu}) \longrightarrow 0
$$

such that $\operatorname{Im} \phi^{\prime}$ is stable by $B$. Hence $B$ induces $B^{\prime}: V\left(\nu^{\prime}\right) \rightarrow V\left(\nu^{\prime}\right)$ and $\bar{B}: V(\bar{\nu}) \rightarrow V(\bar{\nu})$. The maps $q_{1}$ and $q_{2}$ are defined by $q_{1}\left(B, \phi^{\prime}, \bar{\phi}\right)=\left(B^{\prime}, \bar{B}\right)$ and $q_{2}\left(B, \phi^{\prime}, \bar{\phi}\right)=B$, respectively.

For $i \in I$ and $\Lambda \in \operatorname{Irr} \Lambda(\nu)$, set

$$
\varepsilon_{i}(\Lambda)=\varepsilon_{i}(B) \text { and } \varepsilon_{i}^{*}(\Lambda)=\varepsilon_{i}^{*}(B),
$$

where $B$ is a general point of $\Lambda$ and

$$
\begin{aligned}
& \varepsilon_{i}(B)=\operatorname{dim}_{\mathbb{C}} \operatorname{Coker}\left(\underset{\tau ; \operatorname{in}(\tau)=i}{\oplus} V(\nu)_{\text {out }(\tau)} \stackrel{\oplus B_{\mathcal{T}}}{\longrightarrow} V(\nu)_{i}\right),
\end{aligned}
$$

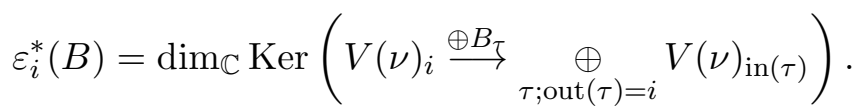

For $k, l \in \mathbb{Z}_{\geq 0}$, we define

$$
(\operatorname{Irr} \Lambda(\nu))_{i, k}=\left\{\Lambda \in \operatorname{Irr} \Lambda(\nu) \mid \varepsilon_{i}(\Lambda)=k\right\} \text { and }(\operatorname{Irr} \Lambda(\nu))_{i}^{l}=\left\{\Lambda \in \operatorname{Irr} \Lambda(\nu) \mid \varepsilon_{i}^{*}(\Lambda)=l\right\} .
$$

Assume $\bar{\nu}=c \alpha_{i}$ (resp. $\left.\nu^{\prime}=c \alpha_{i}\right)$ for $c \in \mathbb{Z}_{\geq 0}$. Since $\Lambda\left(c \alpha_{i}\right)=\{0\}$, we have the following diagrams as special cases of (7.2.1):

$$
\begin{gathered}
\Lambda\left(\nu^{\prime}\right) \cong \Lambda\left(\nu^{\prime}\right) \times \Lambda\left(c \alpha_{i}\right) \stackrel{q_{1}}{\longleftarrow} \Lambda\left(\nu^{\prime}, c \alpha_{i}\right) \stackrel{q_{2}}{\longrightarrow} \Lambda(\nu), \\
\Lambda(\bar{\nu}) \cong \Lambda\left(c \alpha_{i}\right) \times \Lambda(\bar{\nu}) \stackrel{q_{1}}{\longleftarrow} \Lambda\left(c \alpha_{i}, \bar{\nu}\right) \stackrel{q_{2}}{\longrightarrow} \Lambda(\nu) .
\end{gathered}
$$

It is known that the diagrams (7.2.2) and (7.2.3) induce bijections

$$
\widetilde{e}_{i}^{\max }:(\operatorname{Irr} \Lambda(\nu))_{i, c} \stackrel{\sim}{\rightarrow}\left(\operatorname{Irr} \Lambda\left(\nu^{\prime}\right)\right)_{i, 0} \quad \text { and } \quad \widetilde{e}_{i}^{* \max }:(\operatorname{Irr} \Lambda(\nu))_{i}^{c} \stackrel{\sim}{\rightarrow}(\operatorname{Irr} \Lambda(\bar{\nu}))_{i}^{0},
$$

respectively. We introduce maps

$$
\widetilde{e}_{i}, \widetilde{e}_{i}^{*}: \bigsqcup_{\nu \in Q_{+}} \operatorname{Irr} \Lambda(\nu) \rightarrow \bigsqcup_{\nu \in Q_{+}} \operatorname{Irr} \Lambda(\nu) \sqcup\{0\} \quad \text { and } \quad \tilde{f}_{i}, \widetilde{f}_{i}^{*}: \bigsqcup_{\nu \in Q_{+}} \operatorname{Irr} \Lambda(\nu) \rightarrow \bigsqcup_{\nu \in Q_{+}} \operatorname{Irr} \Lambda(\nu)
$$

as follows: if $c>0$ we define

$$
\begin{array}{ll}
\widetilde{e}_{i}:(\operatorname{Irr} \Lambda(\nu))_{i, c} & \stackrel{\sim}{\longrightarrow}\left(\operatorname{Irr} \Lambda\left(\nu^{\prime}\right)\right)_{i, 0} \stackrel{\sim}{\longrightarrow}\left(\operatorname{Irr} \Lambda\left(\nu+\alpha_{i}\right)\right)_{i, c-1}, \\
\widetilde{e}_{i}^{*}:(\operatorname{Irr} \Lambda(\nu))_{i}^{c} & \stackrel{\sim}{\longrightarrow}(\operatorname{Irr} \Lambda(\bar{\nu}))_{i}^{0} \stackrel{\sim}{\longrightarrow}\left(\operatorname{Irr} \Lambda\left(\nu+\alpha_{i}\right)\right)_{i}^{c-1}
\end{array}
$$

and $\widetilde{e}_{i} \Lambda=0$ and $\widetilde{e}_{i}^{*} \Lambda^{\prime}=0$ for $\Lambda \in(\operatorname{Irr} \Lambda(\nu))_{i, 0}$ and $\Lambda^{\prime} \in(\operatorname{Irr} \Lambda(\nu))_{i}^{0}$, respectively. Define

$$
\begin{aligned}
& \tilde{f}_{i}: \quad(\operatorname{Irr} \Lambda(\nu))_{i, c} \stackrel{\sim}{\longrightarrow}\left(\operatorname{Irr} \Lambda\left(\nu^{\prime}\right)\right)_{i, 0} \stackrel{\sim}{\longrightarrow}\left(\operatorname{Irr} \Lambda\left(\nu-\alpha_{i}\right)\right)_{i, c+1}, \\
& \tilde{f}_{i}^{*}:(\operatorname{Irr} \Lambda(\nu))_{i}^{c} \stackrel{\sim}{\longrightarrow}(\operatorname{Irr} \Lambda(\bar{\nu}))_{i}^{0} \stackrel{\sim}{\longrightarrow}\left(\operatorname{Irr} \Lambda\left(\nu-\alpha_{i}\right)\right)_{i}^{c+1} .
\end{aligned}
$$

Theorem 7.2.1. [KS] (1) For $\Lambda \in \operatorname{Irr} \Lambda(\nu)$, we set wt $\Lambda=-\nu, \varphi_{i}(\Lambda)=\varepsilon_{i}(\Lambda)+\left\langle h_{i}\right.$, wt $\left.\Lambda\right\rangle$. Then $\left(\bigsqcup_{\nu \in Q_{+}} \operatorname{Irr} \Lambda(\nu)\right.$; wt, $\left.\varepsilon_{i}, \varphi_{i}, \widetilde{e}_{i}, \widetilde{f}_{i}\right)$ is a crystal isomorphic to $\left(B(\infty)\right.$; wt, $\left.\varepsilon_{i}, \varphi_{i}, \widetilde{e}_{i}, \widetilde{f}_{i}\right)$. More precisely, the explicit form of the isomorphism $\Phi: B(\infty) \stackrel{\sim}{\rightarrow} \bigsqcup_{\nu \in Q_{+}} \operatorname{Irr} \Lambda(\nu)$ is given by $\Phi=\Psi_{\mathbf{i}} \circ \Xi_{\mathbf{i}}^{-1}$. 
(2) Set $\varphi_{i}^{*}(\Lambda)=\varepsilon_{i}^{*}(\Lambda)+\left\langle h_{i}, \mathrm{wt} \Lambda\right\rangle$. Then $\left(\bigsqcup_{\nu \in Q_{+}} \operatorname{Irr} \Lambda(\nu) ; \mathrm{wt}, \varepsilon_{i}^{*}, \varphi_{i}^{*}, \widetilde{e}_{i}^{*}, \widetilde{f}_{i}^{*}\right)$ is a crystal and the bijection $\Phi$ gives an isomorphism of crystals form $\left(B(\infty) ; \mathrm{wt}, \varepsilon_{i}^{*}, \varphi_{i}^{*}, \widetilde{e}_{i}^{*}, \widetilde{f}_{i}^{*}\right)$ to it.

Remark . Because of (7.1.1), the definition of the map $\Phi: B(\infty) \stackrel{\sim}{\rightarrow} \bigsqcup_{\nu \in Q_{+}} \operatorname{Irr} \Lambda(\nu)$ is independent of the choice of $\mathbf{i}$.

7.3. A proof of Lemma 5.6.5. We only show the second formula:

$$
\tilde{f}_{i}^{*}(\mathbf{M}(\mathbf{a}))=\mathbf{M}\left(\tilde{f}_{i}^{*} \mathbf{a}\right) \quad(\mathbf{a} \in \mathcal{B}),
$$

because the first one is proved by similar method.

By Corollary 5.4.1, it is enough to show that

$$
\begin{gathered}
M_{[1, i]^{c}}\left(\tilde{f}_{i}^{*} \mathbf{a}\right)=M_{[1, i]^{c}}(\mathbf{a})-1, \\
M_{K}\left(\widetilde{f}_{i}^{*} \mathbf{a}\right)=M_{K}(\mathbf{a}) \text { for all } K \in \mathcal{M}_{n}^{\times} \backslash \mathcal{M}_{n}^{\times}(i)^{*} .
\end{gathered}
$$

It is easy to see (7.3.1). Indeed, as in the proof of Lemma 5.6.4, we have

$$
\operatorname{wt}(\mathbf{a})=\operatorname{wt}(\mathbf{M}(\mathbf{a}))=\sum_{i \in I} M_{[1, i]^{c}}(\mathbf{a}) \alpha_{i} .
$$

Similarly we have

$$
\mathrm{wt}\left(\tilde{f}_{i}^{*} \mathbf{a}\right)=\sum_{i \in I} M_{[1, i]^{c}}\left(\tilde{f}_{i}^{*} \mathbf{a}\right) \alpha_{i} .
$$

Since $\mathcal{B}$ is a $*$-crystal, we have $\operatorname{wt}\left(\tilde{f}_{i}^{*} \mathbf{a}\right)=\operatorname{wt}(\mathbf{a})-\alpha_{i}$. Therefore (7.3.1) holds.

We shall prove (7.3.2). Assume $K \in \mathcal{M}_{n}^{\times} \backslash \mathcal{M}_{n}^{\times}(i)^{*}$. Namely, $i \in K$ or $i+1 \notin K$. By (7.1.2) and Theorem 7.2.1, it is enough to prove that $M_{K}\left(\widetilde{f}_{i}^{*} \Lambda_{\mathbf{a}}\right)=M_{K}\left(\Lambda_{\mathbf{a}}\right)$ for $i \in K$ or $i+1 \notin K$. Moreover, since $\widetilde{e}_{i}^{* \max }\left(\widetilde{f}_{i}^{*} \Lambda_{\mathbf{a}}\right)=\widetilde{e}_{i}^{* \max } \Lambda_{\mathbf{a}}$, it is enough to show that

$$
M_{K}\left(\widetilde{e}_{i}^{* \max } \Lambda_{\mathbf{a}}\right)=M_{K}\left(\Lambda_{\mathbf{a}}\right) \quad(i \in K \text { or } i+1 \notin K) .
$$

Assume $i \in K_{m}=\left[s_{m}+1, t_{m}\right] \subset K$. Then, there are the following three cases; (a) $m=1$ and $s_{1}=1$, (b) $m=l$ and $t_{m}=n+1$, (c) otherwise. In the case (a), there is the path $\sigma\left(1 \leftarrow t_{1}\right)$ from $t_{1}$ to 1 in $\Omega(K)$ which is is trough $i$. Since 1 (the end point of this path) is not an element of in $(K)$, this path does not appear in the definition of $M_{K}(\Lambda)$ for any $\Lambda$. Therefore $M_{K}\left(\widetilde{e}_{i}^{* \max } \Lambda_{\mathbf{a}}\right)=M_{K}\left(\Lambda_{\mathbf{a}}\right)$. By the similar way, we have (7.3.3) in the case (b).

Let us consider the case (c). In this case, the path $\sigma\left(s_{m} \leftarrow t_{m}\right)$ in $\Omega(K)$ is trough $i$ and $s_{m} \in \operatorname{in}(K), t_{m} \in \operatorname{out}(K)$. We remark that $s_{m}<i$ since $i \in K$. For simplicity, we denote $\Lambda=\Lambda_{\mathbf{a}}$ and $\bar{\Lambda}=\widetilde{e}_{i}^{* \max } \Lambda_{\mathbf{a}}$. Let $\nu=\operatorname{wt}(\Lambda)$ and $\bar{\nu}=\operatorname{wt}(\bar{\Lambda})$, respectively. Take a general point $\bar{B}=\left(\bar{B}_{\tau}\right)_{\tau \in H} \in \bar{\Lambda}$. Recall the diagram (7.2.3) and take a general point $B=\left(B_{\tau}\right)_{\tau \in H} \in \Lambda(\nu)$ of $q_{2} \circ q_{1}^{-1}(B)$. Then $B$ is a general point of $\Lambda$. By the constriction we have the following commutative diagram:

$$
\begin{aligned}
& \bar{\phi}_{t_{m}} \\
& V_{t_{m}}(\nu) \stackrel{\sim}{\rightarrow} V_{t_{m}}(\bar{\nu}) \\
& B_{\sigma\left(i \leftarrow t_{m}\right)} \downarrow \quad \bar{\phi}_{i} \quad \downarrow \bar{B}_{\sigma\left(i \leftarrow t_{m}\right)} \\
& V_{i}(\nu) \rightarrow V_{i}(\bar{\nu}) \\
& B_{\sigma\left(s_{m} \leftarrow i\right)} \downarrow \quad \bar{\phi}_{s_{m}} \downarrow \bar{B}_{\sigma\left(s_{m} \leftarrow i\right)} \\
& V_{s_{m}}(\nu) \stackrel{\sim}{\rightarrow} V_{s_{m}}(\bar{\nu}) \text {. }
\end{aligned}
$$


Therefore we have

$$
\operatorname{Im}\left(B_{\sigma\left(s_{m} \leftarrow t_{m}\right)}\right)=\operatorname{Im}\left(\bar{B}_{\sigma\left(s_{m} \leftarrow t_{m}\right)}\right)
$$

and this formula tells us (7.3.3) holds.

For the case of $i+1 \notin K$, we have (7.3.3) by the similar method. Thus we complete a proof of Lemma 5.6.5.

\section{A NeW Proof of the Anderson-Mirković COnjecture}

8.1. Reformulation of the Anderson-Mirković conjecture. Let us denote $\Lambda=\Lambda_{\mathbf{a}}$. Then Corollary 5.5 .2 can be written as

$$
\left(\widetilde{f}_{i}^{*} \mathbf{M}(\Lambda)\right)_{K}= \begin{cases}\min \left\{M_{K}(\Lambda), M_{s_{i} K}(\Lambda)+c_{i}^{*}(\mathbf{M}(\Lambda))\right\} & \left(K \in \mathcal{M}_{n}^{\times}(i)^{*}\right), \\ M_{K}(\Lambda) & \text { (otherwise) } .\end{cases}
$$

Here $c_{i}^{*}(\mathbf{M}(\Lambda))=M_{[1, i]^{c}}(\Lambda)-M_{([1, i+1] \backslash\{i\})^{c}}(\Lambda)-1$. By Lemma 5.6.5, we already know that

$$
\left(\tilde{f}_{i}^{*} \mathbf{M}(\Lambda)\right)_{K}=M_{K}\left(\tilde{f}_{i}^{*} \Lambda\right) \quad \text { for } K \in \mathcal{M}_{n}^{\times}
$$

Moreover, by (7.3.2), we have

$$
\left(\tilde{f}_{i}^{*} \mathbf{M}(\Lambda)\right)_{K}=M_{K}(\Lambda) \quad \text { for } K \in \mathcal{M}_{n}^{\times} \backslash \mathcal{M}_{n}^{\times}(i)^{*} .
$$

Therefore it is enough to show

$$
M_{K}\left(\widetilde{f}_{i}^{*} \Lambda\right)=\min \left\{M_{K}(\Lambda), M_{s_{i} K}(\Lambda)+c_{i}^{*}(\mathbf{M}(\Lambda))\right\} \text { for } K \in \mathcal{M}_{n}^{\times}(i)^{*} .
$$

Lemma 8.1.1. The formula (8.1.1) is equivalent to the following:

$$
M_{K}(\Lambda)=\min \left\{M_{K}(\bar{\Lambda}), M_{s_{i} K}(\bar{\Lambda})+\left\langle h_{i}, \operatorname{wt}(\bar{\Lambda})\right\rangle-\varepsilon_{i}^{*}(\Lambda)\right\} \text { for } K \in \mathcal{M}_{n}^{\times}(i)^{*} .
$$

Here $\bar{\Lambda}=\widetilde{e}_{i}^{* \max } \Lambda$.

Remark . The formula (8.1.2) is a generalization of the formula which appears in our previous paper [KS].

Consider the case of $K=[1, i+1] \backslash\{i\}(1 \leq i \leq n)$. Then we have

$$
\operatorname{out}(K)=\left\{\begin{array}{ll}
\{2\} & (i=1), \\
\{i-1, i+1\} & (2 \leq i \leq n-1), \\
\{n-1\} & (i=n)
\end{array} \text { and } \operatorname{in}(K)=\{i\} .\right.
$$

Therefore we have

$$
\begin{aligned}
M_{[1, i+1] \backslash\{i\}}(\Lambda) & =-\operatorname{dim}_{\mathbb{C}} \operatorname{Coker}\left(\underset{\tau ; \operatorname{in}(\tau)=i}{\oplus} V(\nu)_{\text {out }(\tau)} \stackrel{\oplus B_{\mathcal{T}}}{\longrightarrow} V(\nu)_{i}\right) \\
& =-\varepsilon_{i}(\Lambda) .
\end{aligned}
$$

Here $B=\left(B_{\tau}\right)$ is a general point of $\Lambda$. Since $s_{i} K=[1, i]$, the formula (8.1.2) is equivalent to

$$
\varepsilon_{i}(\Lambda)=\max \left\{\varepsilon_{i}(\bar{\Lambda}),-\left\langle h_{i}, \operatorname{wt}(\bar{\Lambda})\right\rangle+\varepsilon_{i}^{*}(\Lambda)\right\} .
$$

This is nothing but the formula which appears in [KS], Proposition 5.3.1, (1).

Proof of Lemma 8.1.1. Let $\nu, \bar{\nu}, B, \bar{B}$ be as same as in the proof of Lemma 5.6.5. For simplicity, we denote $\oplus_{i \in I} V_{i}=\oplus_{i \in I} V_{i}(\nu)$ and $\oplus_{i \in I} \bar{V}_{i}=\oplus_{i \in I} V_{i}(\bar{\nu})$.

Before proving the equivalence, we shall show

$$
c_{i}^{*}(\mathbf{M}(\Lambda))=\left\langle h_{i}, \operatorname{wt}(\bar{\Lambda})\right\rangle-\varepsilon_{i}^{*}(\Lambda)-1 .
$$

Since

$$
M_{[1, i]^{c}}(\Lambda)=-\operatorname{dim}_{\mathbb{C}} V_{i},
$$


we have

$$
M_{([1, i+1] \backslash\{i\})^{c}}(\Lambda)=-\operatorname{dim}_{\mathbb{C}} \operatorname{Coker}\left(V_{i} \rightarrow \underset{\tau ; \operatorname{out}(\tau)=i}{\oplus} V_{\operatorname{in}(\tau)}\right),
$$

$$
\begin{aligned}
M_{[1, i]^{c}} & (\Lambda)-M_{([1, i+1] \backslash\{i\})^{c}}(\Lambda) \\
& =-\operatorname{dim}_{\mathbb{C}} V_{i}+\operatorname{dim}_{\mathbb{C}} \operatorname{Coker}\left(V_{i} \rightarrow \underset{\tau ; \operatorname{out}(\tau)=i}{\oplus} V_{\operatorname{in}(\tau)}\right) \\
& =-2 \operatorname{dim}_{\mathbb{C}} V_{i}+\operatorname{dim}_{\mathbb{C}}\left(\underset{\tau ; \operatorname{out}(\tau)=i}{\oplus} V_{\operatorname{in}(\tau)}\right)+\operatorname{dim}_{\mathbb{C}} \operatorname{Ker}\left(V_{i} \rightarrow \underset{\tau ; \operatorname{out}(\tau)=i}{\oplus} V_{\operatorname{in}(\tau)}\right) \\
& =\left\langle h_{i}, \operatorname{wt}(\Lambda)\right\rangle+\varepsilon_{i}^{*}(\Lambda) .
\end{aligned}
$$

Moreover, since

we have

$$
\operatorname{dim}_{\mathbb{C}} \bar{V}_{k}= \begin{cases}\operatorname{dim}_{\mathbb{C}} V_{k} & (k \neq i) \\ \operatorname{dim}_{\mathbb{C}} V_{i}-\varepsilon_{i}^{*}(\Lambda) & (k=i)\end{cases}
$$

$$
\begin{aligned}
c_{i}^{*}(\mathbf{M}(\Lambda)) & =\left\langle h_{i}, \operatorname{wt}(\Lambda)\right\rangle+\varepsilon_{i}^{*}(\Lambda)-1 \\
& =\left\langle h_{i}, \operatorname{wt}(\bar{\Lambda})-\varepsilon_{i}^{*}(\Lambda) \alpha_{i}\right\rangle+\varepsilon_{i}^{*}(\Lambda)-1 \\
& =\left\langle h_{i}, \operatorname{wt}(\bar{\Lambda})\right\rangle-\varepsilon_{i}^{*}(\Lambda)-1 .
\end{aligned}
$$

Thus, (8.1.3) is proved.

Let us prove the equivalence. Firstly, we will show (8.1.1) $\Rightarrow$ (8.1.2). Applying (8.1.1) for $\Lambda_{1}=\widetilde{e}_{i}^{*} \Lambda$, we have

$$
\begin{aligned}
M_{K}(\Lambda) & =M_{K}\left(\tilde{f}_{i}^{*} \Lambda_{1}\right) \\
& =\min \left\{M_{K}\left(\Lambda_{1}\right), M_{s_{i} K}\left(\Lambda_{1}\right)+c_{i}^{*}\left(\mathbf{M}\left(\Lambda_{1}\right)\right)\right\} .
\end{aligned}
$$

Since the vertex $i$ is a source in $\Omega\left(s_{i} K\right)$ and $\overline{\Lambda_{1}}=\bar{\Lambda}$, we have

$$
M_{s_{i} K}\left(\Lambda_{1}\right)=M_{s_{i} K}\left(\overline{\Lambda_{1}}\right)=M_{s_{i} K}(\bar{\Lambda}) \text {. }
$$

On the other hand,

$$
\begin{aligned}
c_{i}^{*}\left(\mathbf{M}\left(\Lambda_{1}\right)\right) & =\left\langle h_{i}, \operatorname{wt}\left(\overline{\Lambda_{1}}\right)\right\rangle-\varepsilon_{i}^{*}\left(\Lambda_{1}\right)-1 \\
& =\left\langle h_{i}, \operatorname{wt}(\bar{\Lambda})\right\rangle-\varepsilon_{i}^{*}(\Lambda) .
\end{aligned}
$$

Therefore we have

$$
M_{K}(\Lambda)=\min \left\{M_{K}\left(\Lambda_{1}\right), M_{s_{i} K}(\bar{\Lambda})+\left\langle h_{i}, \operatorname{wt}(\bar{\Lambda})\right\rangle-\varepsilon_{i}^{*}(\Lambda)\right\} .
$$

Similarly, applying (8.1.1) for $\Lambda_{2}=\widetilde{e}_{i}^{*} \Lambda_{1}=\left(\widetilde{e}_{i}^{*}\right)^{2} \Lambda$, we have

$$
\begin{aligned}
M_{K}\left(\Lambda_{1}\right) & =\min \left\{M_{K}\left(\Lambda_{2}\right), M_{s_{i} K}(\bar{\Lambda})+\left\langle h_{i}, \operatorname{wt}(\bar{\Lambda})\right\rangle-\varepsilon_{i}^{*}\left(\Lambda_{1}\right)\right\} \\
& =\min \left\{M_{K}\left(\Lambda_{2}\right), M_{s_{i} K}(\bar{\Lambda})+\left\langle h_{i}, \operatorname{wt}(\bar{\Lambda})\right\rangle-\varepsilon_{i}^{*}(\Lambda)+1\right\} .
\end{aligned}
$$

By substituting this formula for (8.1.4), we have

$$
\begin{gathered}
M_{K}(\Lambda)=\min \left\{\min \left\{M_{K}\left(\Lambda_{2}\right), M_{s_{i} K}(\bar{\Lambda})+\left\langle h_{i}, \operatorname{wt}(\bar{\Lambda})\right\rangle-\varepsilon_{i}^{*}(\Lambda)+1\right\},\right. \\
\left.M_{s_{i} K}(\bar{\Lambda})+\left\langle h_{i}, \operatorname{wt}(\bar{\Lambda})\right\rangle-\varepsilon_{i}^{*}(\Lambda)\right\} \\
=\min \left\{M_{K}\left(\Lambda_{2}\right), M_{s_{i} K}(\bar{\Lambda})+\left\langle h_{i}, \operatorname{wt}(\bar{\Lambda})\right\rangle-\varepsilon_{i}^{*}(\Lambda)\right\} .
\end{gathered}
$$

After repeating the similar method, we have

$$
M_{K}(\Lambda)=\min \left\{M_{K}(\bar{\Lambda}), M_{s_{i} K}(\bar{\Lambda})+\left\langle\alpha_{i}, \operatorname{wt}(\bar{\Lambda})\right\rangle-\varepsilon_{i}^{*}(\Lambda)\right\} .
$$


This is nothing but the formula (8.1.2).

Secondly let us prove $(8.1 .2) \Rightarrow(8.1 .1)$. By (8.1.2) for $\widetilde{f}_{i}^{*} \Lambda$ and $\overline{\tilde{f}_{i}^{*} \Lambda}=\bar{\Lambda}$, we have

$$
\begin{aligned}
M_{K}\left(\widetilde{f}_{i}^{*} \Lambda\right) & =\min \left\{M_{K}\left(\overline{\tilde{f}_{i}^{*} \Lambda}\right), M_{s_{i} K}\left(\overline{\tilde{f}_{i}^{*} \Lambda}\right)+\left\langle\alpha_{i}, \operatorname{wt}\left(\overline{\tilde{f}_{i}^{*} \Lambda}\right)\right\rangle-\varepsilon_{i}^{*}\left(\tilde{f}_{i}^{*} \Lambda\right)\right\} \\
& =\min \left\{M_{K}(\bar{\Lambda}), M_{s_{i} K}(\bar{\Lambda})+\left\langle h_{i}, \operatorname{wt}(\bar{\Lambda})\right\rangle-\varepsilon_{i}^{*}(\Lambda)-1\right\} .
\end{aligned}
$$

Since $M_{s_{i} K}(\bar{\Lambda})+\left\langle h_{i}, \operatorname{wt}(\bar{\Lambda})\right\rangle-\varepsilon_{i}^{*}(\Lambda)>M_{s_{i} K}(\bar{\Lambda})+\left\langle h_{i}, \operatorname{wt}(\bar{\Lambda})\right\rangle-\varepsilon_{i}^{*}(\Lambda)-1$,

the right hand side $=\min \left\{\min \left\{M_{K}(\bar{\Lambda}), M_{s_{i} K}(\bar{\Lambda})+\left\langle h_{i}, \operatorname{wt}(\bar{\Lambda})\right\rangle-\varepsilon_{i}^{*}(\Lambda)\right\}\right.$,

$$
\begin{aligned}
& \left.M_{s_{i} K}(\bar{\Lambda})+\left\langle h_{i}, \operatorname{wt}(\bar{\Lambda})\right\rangle-\varepsilon_{i}^{*}(\Lambda)-1\right\} \\
= & \min \left\{M_{K}(\Lambda), M_{s_{i} K}(\bar{\Lambda})+\left\langle h_{i}, \operatorname{wt}(\bar{\Lambda})\right\rangle-\varepsilon_{i}^{*}(\Lambda)-1\right\} \\
= & \min \left\{M_{K}(\Lambda), M_{s_{i} K}(\bar{\Lambda})+c_{i}^{*}(\mathbf{M}(\Lambda))\right\} .
\end{aligned}
$$

Because $s_{i} K \in \mathcal{M}_{n}^{\times} \backslash \mathcal{M}_{n}^{\times}(i)^{*}$, we have $M_{s_{i} K}(\bar{\Lambda})=M_{s_{i} K}(\Lambda)$ by (7.3.3). Therefore we have

$$
M_{K}\left(\widetilde{f}_{i}^{*} \Lambda\right)=\min \left\{M_{K}(\Lambda), M_{s_{i} K}(\Lambda)+c_{i}^{*}(\mathbf{M}(\Lambda))\right\} .
$$

This is nothing but (8.1.1).

8.2. A proof of the formula (8.1.2). The aim of this subsection is to prove the next proposition:

Proposition 8.2.1. The formula (8.1.2) holds for any $K \in \mathcal{M}_{n}^{\times}(i)^{*}$.

Set

$$
\bar{W}_{s_{i} K}=\operatorname{Ker}\left(\underset{p \in \operatorname{out}\left(s_{i} K\right)}{\oplus} \bar{V}_{p} \stackrel{\oplus \bar{B}_{p \rightarrow q}}{\longrightarrow} \underset{q \in \operatorname{in}\left(s_{i} K\right)}{\oplus} \bar{V}_{q}\right) \subset\left(\underset{p \in \operatorname{out}\left(s_{i} K\right)}{\oplus} \bar{V}_{p}\right) .
$$

By the assumption, we have

$$
\operatorname{out}\left(s_{i} K\right) \backslash\{i\} \subset \operatorname{out}(K) \subset\left(\operatorname{out}\left(s_{i} K\right) \backslash\{i\}\right) \cup\{i-1, i+1\} .
$$

Therefore we can define a map $\Phi: \bar{W}_{s_{i} K} \rightarrow\left(\oplus_{k \in \operatorname{out}(K)} V_{k}\right)$ by

$$
\Phi\left(\sum_{p \in \operatorname{Out}\left(s_{i} K\right)} \bar{w}_{p}\right)=\delta_{K}(i-1) \bar{B}_{i \rightarrow i-1}\left(\bar{w}_{i}\right)+\delta_{K}(i+1) \bar{B}_{i \rightarrow i+1}\left(\bar{w}_{i}\right)+\sum_{p \in \operatorname{Out}\left(s_{i} K\right) \backslash\{i\}} \bar{w}_{p},
$$

where $\bar{w}_{p} \in \bar{V}_{p}$ and $\delta_{K}$ is a map form $[1, n]$ to $\{0,1\}$ defined by

$$
\delta_{K}(k)= \begin{cases}1 & (k \in \operatorname{out}(K)) \\ 0 & \text { (otherwise) }\end{cases}
$$

Here we remark that, if $p \in \operatorname{out}\left(s_{i} K\right) \backslash\{i\}$, we have $p \in \operatorname{out}(K)$ and $\bar{V}_{p}=V_{p}$.

Set

$$
N=\operatorname{Coker}(\Phi)
$$

and consider a map

$$
\widetilde{\mathrm{Id}}:\left(\underset{k \in \operatorname{out}(K)}{\oplus} V_{k}\right) \rightarrow N
$$


which is naturally induced form the identity map Id : $\left(\underset{k \in \operatorname{out}(K)}{\oplus} V_{k}\right) \stackrel{\sim}{\rightarrow}\left(\underset{k \in \operatorname{out}(K)}{\oplus} V_{k}\right)$.

By the construction, it is clear that $\widetilde{\text { Id }}$ is surjective.

In the above setting, the following two Lemmas hold:

Lemma 8.2.2. $\Phi$ is injective.

Lemma 8.2.3. Let $k \in \operatorname{out}(K), l \in \operatorname{in}(K)$ and assume that there is a path $\sigma(k \rightarrow l)$ in the orientation $\Omega(K)$.

(1) If $l \neq i$, then the map $B_{\sigma(k \rightarrow l)}: V_{k} \rightarrow V_{l}\left(=\bar{V}_{l}\right)$ induces a map $\psi_{l}: N \rightarrow V_{l}$ such that

$$
B_{\sigma(k \rightarrow l)}\left(=\bar{B}_{\sigma(k \rightarrow l)}\right)=\psi_{l} \circ \widetilde{I} d .
$$

(2) If $l=i$, then the map $B_{\sigma(k \rightarrow i)}: V_{k} \rightarrow \bar{V}_{i}\left(\neq V_{i}\right)$ induces a map $\psi_{i}: N \rightarrow \bar{V}_{i}$ such that

$$
\bar{B}_{\sigma(k \rightarrow i)}=\psi_{i} \circ \widetilde{I} d \text {. }
$$

Moreover, let $\pi_{i}: V_{i} \rightarrow \bar{V}_{i}$ be the natural projection and $\varphi_{i}: N \rightarrow V_{i}$ a generic map such that $\psi_{i}=\pi_{i} \circ \varphi_{i}$. Then we have

$$
B_{\sigma(k \rightarrow i)}=\varphi_{i} \circ \widetilde{I} d .
$$

The above two lemmas are easy exercises on linear algebra. So, we omit to give proofs.

Proof of Proposition 8.2.1. Since $\Phi$ is injective, we have

$$
\begin{aligned}
& \operatorname{dim}_{\mathbb{C}} N=\sum_{k \in \operatorname{out}(K)} \operatorname{dim}_{\mathbb{C}} V_{k}-\operatorname{dim}_{\mathbb{C}} \bar{W}_{s_{i} K} \\
& =\sum_{k \in \operatorname{out}(K)} \operatorname{dim}_{\mathbb{C}} V_{k}-\operatorname{dim}_{\mathbb{C}} \operatorname{Ker}\left(\underset{p \in \operatorname{out}\left(s_{i} K\right)}{\oplus} \bar{V}_{p} \stackrel{\oplus \bar{B}_{\sigma(p \rightarrow q)}}{\longrightarrow} \underset{q \in \operatorname{in}\left(s_{i} K\right)}{\oplus} \bar{V}_{q}\right) \\
& =\sum_{k \in \operatorname{out}(K)} \operatorname{dim}_{\mathbb{C}} V_{k}-\sum_{p \in \operatorname{out}\left(s_{i} K\right)} \operatorname{dim}_{\mathbb{C}} \bar{V}_{p}+\sum_{q \in \operatorname{in}\left(s_{i} K\right)} \operatorname{dim}_{\mathbb{C}} \bar{V}_{q} \\
& -\operatorname{dim}_{\mathbb{C}} \operatorname{Coker}\left(\underset{p \in \operatorname{out}\left(s_{i} K\right)}{\oplus} \bar{V}_{p} \stackrel{\oplus \bar{B}_{\sigma(p \rightarrow q)}}{\longrightarrow} \underset{q \in \operatorname{in}\left(s_{i} K\right)}{\oplus} \bar{V}_{q}\right) \\
& =\sum_{k \in \operatorname{out}(K)} \operatorname{dim}_{\mathbb{C}} V_{k}-\sum_{p \in \operatorname{out}\left(s_{i} K\right)} \operatorname{dim}_{\mathbb{C}} \bar{V}_{p}+\sum_{q \in \operatorname{in}\left(s_{i} K\right)} \operatorname{dim}_{\mathbb{C}} \bar{V}_{q}+M_{s_{i} K}(\bar{\Lambda}) .
\end{aligned}
$$

Denote the direct sum of the maps $\psi_{l}\left(\right.$ resp. $\left.\varphi_{l}\right)(l \in$ in $(\mathrm{K}))$ by

$$
\psi=\underset{l \in \operatorname{in}(K)}{\oplus} \psi_{l}: N \rightarrow \underset{l \in \operatorname{in}(K)}{\oplus} \bar{V}_{l} \quad\left(\text { resp. } \varphi=\underset{l \in \operatorname{in}(K)}{\oplus} \varphi_{l}: N \rightarrow \underset{l \in \operatorname{in}(K)}{\oplus} V_{l}\right) .
$$

Here we set $\varphi_{l}=\psi_{l}$ for $l \neq i$.

By the definition, we have

$$
\operatorname{Im}\left(\underset{k \in \operatorname{out}(K)}{\oplus} V_{k} \stackrel{\oplus B_{\sigma(k \rightarrow l)}}{\longrightarrow} \underset{l \in \operatorname{in}(K)}{\oplus} V_{l}\right)=\operatorname{Im}\left(N \stackrel{\varphi}{\longrightarrow} \underset{l \in \operatorname{in}(K)}{\oplus} V_{l}\right) .
$$

Moreover, by the genericity of $\varphi$, we have

$$
\operatorname{dim} \operatorname{Ker} \varphi=\max \left\{\operatorname{dim}_{\mathbb{C}} \operatorname{Ker} \psi-\varepsilon_{i}^{*}(\Lambda), 0\right\} .
$$


Combining the above results, we have

$$
-M_{K}(\Lambda)=\sum_{l \in \operatorname{in}(K)} \operatorname{dim}_{\mathbb{C}} V_{l}-\operatorname{dim}_{\mathbb{C}} N+\max \left\{\operatorname{dim}_{\mathbb{C}} \operatorname{Ker} \psi-\varepsilon_{i}^{*}(\Lambda), 0\right\} .
$$

Indeed,

$$
\begin{aligned}
-M_{K}(\Lambda) & =\operatorname{dim}_{\mathbb{C}} \operatorname{Coker}\left(\underset{k \in \operatorname{out}(K)}{\oplus} V_{k} \stackrel{\oplus B_{\sigma(k \rightarrow l)}}{\longrightarrow} \underset{l \in \operatorname{in}(K)}{\oplus} V_{l}\right) \\
& =\sum_{l \in \operatorname{in}(K)} \operatorname{dim}_{\mathbb{C}} V_{l}-\operatorname{dim}_{\mathbb{C}} \operatorname{Im}\left(\underset{k \in \operatorname{out}(K)}{\oplus} V_{k} \stackrel{\left.\oplus B_{\sigma(k \rightarrow l)} \underset{l \in \operatorname{in}(K)}{\oplus} V_{l}\right)}{\longrightarrow} \sum_{l \in \operatorname{in}(K)}^{\oplus} \operatorname{dim}_{\mathbb{C}} V_{l}-\operatorname{dim}_{\mathbb{C}} \operatorname{Im} \varphi\right. \\
& =\sum_{l \in \operatorname{in}(K)} \operatorname{dim}_{\mathbb{C}} V_{l}-\operatorname{dim}_{\mathbb{C}} N+\operatorname{dim}_{\mathbb{C}} \operatorname{Ker} \varphi \\
& =\sum_{l \in \operatorname{in}(K)} \operatorname{dim}_{\mathbb{C}} V_{l}-\operatorname{dim}_{\mathbb{C}} N+\max \left\{\operatorname{dim}_{\mathbb{C}} \operatorname{Ker} \psi-\varepsilon_{i}^{*}(\Lambda), 0\right\} .
\end{aligned}
$$

Lemma 8.2.4. The following formulas hold:

(1) $\sum_{l \in \operatorname{in}(K)} \operatorname{dim}_{\mathbb{C}} V_{l}-\operatorname{dim}_{\mathbb{C}} N+\operatorname{dim}_{\mathbb{C}} \operatorname{Ker} \psi-\varepsilon_{i}^{*}(\Lambda)=-M_{K}(\bar{\Lambda})$,

(2) $\sum_{l \in \operatorname{in}(K)} \operatorname{dim}_{\mathbb{C}} V_{l}-\operatorname{dim}_{\mathbb{C}} N=-M_{s_{i} K}(\bar{\Lambda})+\varepsilon_{i}^{*}(\Lambda)-\left\langle h_{i}, \operatorname{wt}(\bar{\Lambda})\right\rangle$.

Proof. The formula (1) is proved by a direct computation. Indeed, we have

$$
\begin{aligned}
\sum_{l \in \operatorname{in}(K)} \operatorname{dim}_{\mathbb{C}} V_{l}-\operatorname{dim}_{\mathbb{C}} N+\operatorname{dim}_{\mathbb{C}} \operatorname{Ker} \psi-\varepsilon_{i}^{*}(\Lambda) & \\
& =\sum_{l \in \operatorname{in}(K)} \operatorname{dim}_{\mathbb{C}} \bar{V}_{l}-\operatorname{dim}_{\mathbb{C}} \operatorname{Im} \psi \\
& =\sum_{l \in \operatorname{in}(K)} \operatorname{dim}_{\mathbb{C}} \bar{V}_{l}-\operatorname{dim}_{\mathbb{C}} \operatorname{Im}\left(\underset{k \in \operatorname{out}(K)}{\oplus} \bar{V}_{k} \stackrel{\left.\oplus \bar{B}_{\sigma(k \rightarrow l)} \underset{l \in \operatorname{in}(K)}{\oplus} \bar{V}_{l}\right)}{\longrightarrow}\right. \\
& =\operatorname{dim}_{\mathbb{C}} \operatorname{Coker}\left(\underset{k \in \operatorname{out}(K)}{\oplus} \bar{V}_{k} \stackrel{\left.\oplus \bar{B}_{\sigma(k \rightarrow l)} \underset{l \in \operatorname{in}(K)}{\oplus} \bar{V}_{l}\right)}{=}\right. \\
& =-M_{K}(\bar{\Lambda}) .
\end{aligned}
$$

Let us show the formula (2). We have

$$
\begin{aligned}
\sum_{l \in \operatorname{in}(K)} \operatorname{dim}_{\mathbb{C}} V_{l}-\operatorname{dim}_{\mathbb{C}} N=\sum_{l \in \operatorname{in}(K)} \operatorname{dim}_{\mathbb{C}} V_{l}-\sum_{k \in \operatorname{out}(K)} \operatorname{dim}_{\mathbb{C}} V_{k} \\
+\sum_{p \in \operatorname{out}\left(s_{i} K\right)} \operatorname{dim}_{\mathbb{C}} \bar{V}_{p}-\sum_{q \in \operatorname{in}\left(s_{i} K\right)} \operatorname{dim}_{\mathbb{C}} \bar{V}_{q}-M_{s_{i} K}(\bar{\Lambda}) .
\end{aligned}
$$


Therefore it is enough to show that

$$
\begin{gathered}
\sum_{l \in \operatorname{in}(K)} \operatorname{dim} V_{l}-\sum_{k \in \operatorname{out}(K)} \operatorname{dim} V_{k}+\sum_{p \in \operatorname{out}\left(s_{i} K\right)} \operatorname{dim} \bar{V}_{p}-\sum_{q \in \operatorname{in}\left(s_{i} K\right)} \operatorname{dim} \bar{V}_{q} \\
=\varepsilon_{i}^{*}(\Lambda)-\left\langle h_{i}, \operatorname{wt}(\bar{\Lambda})\right\rangle .
\end{gathered}
$$

But it is easily checked by a direct computation. Thus, we get the formula.

Let us return to the proof of Proposition 8.2.1. Substituting the result of the above lemma for (8.2.1), we get

$$
-M_{K}(\Lambda)=\max \left\{-M_{K}(\bar{\Lambda}),-M_{s_{i} K}(\bar{\Lambda})-\left\langle\alpha_{i}, \operatorname{wt}(\bar{\Lambda})\right\rangle+\varepsilon_{i}^{*}(\Lambda)\right\} .
$$

This is nothing but the formula (8.1.2). Thus we have the statement.

\section{Appendix A. A proof of the formula (7.1.1)}

In this appendix, we will give a proof of the formula (7.1.1).

A.1. Results in [KS]. Let us recall some results in [KS]. Consider two quivers of type $A_{n},(I, \Omega)$ and $\left(I, \Omega^{\prime}\right)$. Assume $\mathbf{i}\left(\right.$ resp. $\left.\mathbf{i}^{\prime}\right)$ is a reduced word of the longest element which is adapted to the orientation $\Omega\left(\right.$ resp. $\left.\Omega^{\prime}\right)$.

Let $\nu \in Q_{+}$and $\mathcal{O}_{\mathbf{a}, \Omega}$ be the $G(\nu)$-orbit in $E_{V(\nu), \Omega}$ corresponding to an i-Lusztig datum $\mathbf{a}$, and $\mathcal{O}_{\mathbf{a}^{\prime}, \Omega^{\prime}}$ the orbit in $E_{V(\nu), \Omega^{\prime}}$ corresponding to an $\mathbf{i}^{\prime}$-Lusztig datum $\mathbf{a}^{\prime}=R_{\mathbf{i}}^{\mathbf{i}^{\prime}}(\mathbf{a})$ where $R_{\mathbf{i}}^{\mathbf{i}^{\prime}}$ is the transition map from $\mathbf{i}$ to $\mathbf{i}^{\prime}$.

Set

$$
\Lambda_{\mathbf{a}}:=\overline{T_{\mathcal{O}_{\mathbf{a}}, \Omega}^{*} E_{V(\nu), \Omega}} \quad \text { and } \quad \Lambda_{\mathbf{a}^{\prime}}:=\overline{T_{\mathcal{O}_{\mathbf{a}^{\prime}}, \Omega^{\prime}}^{*} E_{V(\nu), \Omega^{\prime}}} .
$$

The goal is to prove

$$
\Lambda_{\mathbf{a}}=\Lambda_{\mathbf{a}^{\prime}}
$$

Since our quiver is of type $A_{n}$, there uniquely exists a $G(\nu)$-orbit $\mathcal{O}_{\mathbf{b}, \Omega}$ in $E_{V(\nu), \Omega}$ such that $\Lambda_{\mathbf{a}^{\prime}}=\overline{T_{\mathcal{O}_{\mathbf{b}, \Omega}}^{*} E_{V(\nu), \Omega}}$. (Here we denote by $\mathbf{b}$ the corresponding $\mathbf{i}$-Lusztig datum.) We consider a map

$$
s:\left\{G(\nu) \text {-orbits in } E_{V(\nu), \Omega}\right\} \rightarrow\left\{G(\nu) \text {-orbits in } E_{V(\nu), \Omega}\right\}
$$

defined by $\mathcal{O}_{\mathbf{a}, \Omega} \mapsto \mathcal{O}_{\mathbf{b}, \Omega}$. We remark that, by the definition, $s$ is a bijection. To prove the formula (A.1.1) (or equivalently (7.1.1)), it is enough to show that $s$ is the identity map.

Let $\mathbb{C}_{\mathcal{O}_{\mathbf{a}, \Omega}}$ be the constant sheaf on the orbit $\mathcal{O}_{\mathbf{a}, \Omega},{ }^{\pi} \mathbb{C}_{\mathcal{O}_{\mathbf{a}, \Omega}}$ its minimal extension and $S S\left({ }^{\pi} \mathbb{C}_{\mathcal{O}_{\mathbf{a}, \Omega}}\right)$ its singular support. Then we have

$$
S S\left({ }^{\pi} \mathbb{C}_{\mathcal{O}_{\mathbf{a}, \Omega}}\right) \supset \Lambda_{\mathbf{a}}=\overline{T_{\mathcal{O}_{\mathbf{a}, \Omega}}^{*} E_{V(\nu), \Omega}} .
$$

In addition to the above, the next result is proved by Lusztig:

Theorem A.1.1 ([L1], [L2]).

$$
S S\left({ }^{\pi} \mathbb{C}_{\mathcal{O}_{\mathbf{a}, \Omega}}\right)=S S\left({ }^{\pi} \mathbb{C}_{\mathcal{O}_{\mathbf{a}^{\prime}, \Omega^{\prime}}}\right)
$$

Therefore we have

$$
S S\left({ }^{\pi} \mathbb{C}_{\mathcal{O}_{\mathbf{a}, \Omega}}\right)=S S\left({ }^{\pi} \mathbb{C}_{\mathcal{O}_{\mathbf{a}^{\prime}, \Omega^{\prime}}}\right) \supset \Lambda_{\mathbf{a}^{\prime}}=\overline{T_{\mathcal{O}_{\mathbf{b}, \Omega}}^{*} E_{V(\nu), \Omega}}=\overline{T_{s\left(\mathcal{O}_{\mathbf{a}, \Omega}\right)}^{*} E_{V(\nu), \Omega}}
$$


Remark . In [L2] and [KS], they consider a similar problem in more general setting. Let $\mathfrak{g}$ be an arbitrary symmetric Kac-Moody Lie algebra and $B(\infty)$ the crystal basis of $U_{q}^{-}(\mathfrak{g})$. In $[\mathrm{KS}]$, they define a crystal structure on the set of all irreducible components of Lusztig's quiver varieties, and show that it is isomorphic to $B(\infty)$. For $b \in B(\infty)$, we denote by $\Lambda_{b}$ the corresponding irreducible component. On the other hand, let $\mathbf{B}$ be the Lusztig's canonical basis of $U_{q}^{-}(\mathfrak{g})$. It is constructed as the set of certain simple perverse shaeves on the sepace of representations of a quiver attached to $\mathfrak{g}$ ([L2]). Recall that there is a canonical bijection between $B(\infty)$ and $\mathbf{B}$. For $b \in B(\infty)$, we denote by $L_{b, \Omega}$ the corresponding simple perverse sheaf. Then it is known that

$$
S S\left(L_{b, \Omega}\right) \supset \Lambda_{b} \quad \text { for any } b \in B(\infty) .
$$

Now, let us consider the following problem:

Problem. Assume $s: B(\infty) \rightarrow B(\infty)$ is a bijection such that $S S\left(L_{b, \Omega}\right) \supset \Lambda_{s(b)}$ for any $b \in B(\infty)$. Then, is $s$ the identity?

The above problem was firstly considered by Lusztig ([L2]). In [KS, they stated that "s must be the identity under the above assumption". But their "proof" of the statement is wrong. Therefore, the problem is still open for an arbitrary case.

On the other hand, Kimura $\mathrm{Kim}$ shows that the bijection $s$ must be the identity for finite $A D E$ type cases and cyclic quiver cases. In the next subsection, we will give a proof following $[\mathrm{Kim}]$.

A.2. Kimura's proof of (A.1.1). By $(A .1 .2)$ and the definition of singular supports, we have

$$
\overline{\mathcal{O}_{\mathbf{a}, \Omega}} \supset \mathcal{O}_{\mathbf{b}, \Omega}=s\left(\mathcal{O}_{\mathbf{a}, \Omega}\right) .
$$

Namely, the bijection $s$ preserves closure relations on $G(\nu)$-orbits in $E_{V(\nu), \Omega}$ :

$$
s\left(\mathcal{O}_{\Omega}\right) \subset \overline{\mathcal{O}_{\Omega}} \text { for any } G(\nu) \text { - orbit } \mathcal{O}_{\Omega} \text { in } E_{V(\nu), \Omega} .
$$

Hence, it is enough to prove that the following statement:

Proposition A.2.1 ([Kim $)$. Let $s$ be a bijection on the set of $G(\nu)$-orbits in $E_{V(\nu), \Omega}$ which preserves closure relations. Then s must be the identity.

Let us introduce an ordering $\leq$ on the set of $G(\nu)$-orbits in $E_{V(\nu), \Omega}$ by

$$
\mathcal{O}_{\Omega} \leq \mathcal{O}_{\Omega}^{\prime} \stackrel{\text { def }}{\Longleftrightarrow} \mathcal{O}_{\Omega}^{\prime} \subset \overline{\mathcal{O}_{\Omega}}
$$

By using this ordering, (A.2.1) can be rewritten as:

$$
s\left(\mathcal{O}_{\Omega}\right) \subset \overline{\mathcal{O}_{\Omega}}=\mathcal{O}_{\Omega} \cup \underset{\mathcal{O}_{\Omega}^{\prime}: \mathcal{O}_{\Omega}<\mathcal{O}_{\Omega}^{\prime}}{\bigcup} \mathcal{O}_{\Omega}^{\prime} .
$$

Proof of A.2.1. We will show the statement by the decreasing induction on $\leq$. Note that, by the definition, there is a maximal element with respect to the ordering $\leq$. Let $\mathcal{O}_{\Omega}$ be a such element. Hence, by $(A .2 .2)$ and the maximality of $\mathcal{O}_{\Omega}, s\left(\mathcal{O}_{\Omega}\right)$ must be equal to $\mathcal{O}_{\Omega}$. Assume $s\left(\mathcal{O}_{\Omega}^{\prime}\right)=\mathcal{O}_{\Omega}^{\prime}$ for any $\mathcal{O}_{\Omega}<\mathcal{O}_{\Omega}^{\prime}$. Since $s$ is a bijection, we have $s\left(\mathcal{O}_{\Omega}\right)=\mathcal{O}_{\Omega}$ by (A.2.2).

Remark . For finite $D, E$ cases and cyclic quiver cases, the similar method does work. Namely, we can show that $s$ must be the identity for such cases (see [Kim], in detail). 


\section{REFERENCES}

[A] J. E. Anderson, A polytope calculus for semisimple groups, Duke. Math. J. 116 (2003), 567-588.

[BFZ] A. Berenstein, S. Fomin and A. Zelevinsky, Parametrizations of canonical bases and totally positive matrices, Adv. Math. 122 (1996), 49-149.

[BG] A. Breverman and D. Gaitsgory, Crystals via the affine Grassmannian, Duke. Math. J. 107 (2001), 561-575.

[BK] P. Baumann and J. Kamnitzer, Preprojective algebras and MV polytopes, arXiv:1009.2469.

[EK] N. Enomoto and M. Kashiwara, Symmetric crystals for $\mathfrak{g} l_{\infty}$, Publ. RIMS. 44 (2008), 837-891.

[Kam1] J. Kamnitzer, Mirković-Vilonen cycles and polytopes, Ann. of Math. (2) 171 (2010), no. 1, 245-294.

[Kam2] J. Kamnitzer, The crystal structure on the set of Mirković-Vilonen polytopes, Adv. Math. 215 (2007), 66-93.

[KamS] J. Kamnitzer and C. Sadanand, Modules with 1-dimensional socle and components of Lusztig quiver varieties in type A, arXiv:1009.0272

[K1] M. Kashiwara, Crystallizing the q-analogue of universal enveloping algebras, Duke Math. J. 63 (1991), 465-516.

[K2] M. Kashiwara, Global crystal base of quantum groups, Duke Math. J. 69 (1993), 455-485.

[K3] M. Kashiwara, Crystal base and Littelmann's refined Demazure character formula, Duke Math. J. 71 (1993), 839-858.

[K4] M. Kashiwara, Bases cristallines des groupes quantiques, Cours Spécialisés Vol. 9, Société Mathématique de France, Paris, 2002.

[KS] M. Kashiwara and Y. Saito, Geometric construction of crystal bases, Duke Math. J. 89 (1997), 9-36.

[Kim] Y. Kimura, Affine quivers and crystal bases, Master thesis, Kyoto University (2007).

[Li] P. Littelmann, A Littlewood-Richardson rule for symmetrizable Kac-Moody Lie algebra, Invent. Math. 116 (1994), 329-346.

[L1] G. Lusztig, Canonical bases arising from quantized universal enveloping algebras, J. Amer. Math. Soc. 3 (1990), 447-498.

[L2] G. Lusztig, Quivers, perverse sheaves, and quantized universal enveloping algebras, J. Amer. Math. Soc. 4 (1991), 365-421.

[L3] G. Lusztig, Affine quivers and canonical bases, Publ. Math. IHES 76 (1992), 111-163.

[L4] G. Lusztig, Introduction to quantum groups, Progr. Math. 110 (1993), Birkhäuser.

[MV1] I. Mirković and K. Vilonen, Perverse sheaves on affine Grassmannian and Langlands duality, Math. Res. Lett. 7 (2000), 13-24.

[MV2] I. Mirković and K. Vilonen, Geometric Langlands duality and representations of algebraic groups over commutative rings, Ann. of Math. (2) 166 (2007), 95-143.

[NSS1] S. Naito, D. Sagaki and Y. Saito, Toward Berenstein-Zelevinsky data in affine type A, I: Construction of affine analogs, arXiv:1009.4526.

[NSS2] S. Naito, D.Sagaki and Y. Saito, Toward Berenstein-Zelevinsky data in affine type A, II: Explicit description, arXiv:1101.3621.

[NZ] T. Nakashima and A. Zelevinsky, Polyhedral realizations of crystal bases for quantized KacMoody algebras, Adv. Math. 131 (1997), 253-278.

[Re] M. Reineke, On the coloured graph structure of Lusztig's canonical basis, Math. Ann. 307 (1997), 705-723.

[S] Y. Saito, PBW basis of quantum universal enveloping algebras, Publ. RIMS. 30 (1994), 209-232.

[Sav] A. Savage, Geometric and combinatorial realization of crystal graphs, Algebr. Rep. Theory 9 (2006), 161-199.

Graduate School of Mathematical Sciences, University of Tokyo, 3-8-1 Komaba, MeguroKU, TOKYO 153-8914, JAPAN.

E-mail address: yosihisa@ms.u-tokyo.ac.jp 